\title{
Judging the Misunderstood: A Pilot Public Stigma Intervention Towards Non-Offending Paedophilia
}

\author{
Ross Keegan, BSc
}

This research was conducted under the supervision of Dr Guillaume Roussellete at the University of Glasgow. The primary author freely chose to pursue the topic and has every intention of continuing to conduct research in this area.

The author has attained graduate basis for chartered membership in the British Psychological Society following completion of a Psychology Undergraduate Degree. They have a commitment to transparency in research and as such the data along with the code utilised in this research is available online. The development and utilisation of more robust statistical analysis methods along with the research and development of widescale public interventions to increase participation of nonoffending paedophiles in formal help services are the primary academic interests of the author. 


\title{
Judging the Misunderstood: A Pilot Public Stigma Intervention Towards Non-Offending Paedophilia
}

\begin{abstract}
Public stigmatisation has been identified as a barrier to seeking therapy amongst people with paedophilia (PWP). This study piloted a public stigmatism intervention towards non-offending paedophilia and compared its effectiveness with a public stigmatism intervention towards alcoholism. The overall aim was to reduce self-reported stigmatism towards PWP and assess the comparative effectiveness of the stigmatism intervention towards PWP with the alcoholism stigmatism intervention.
\end{abstract}

A 2x2 mixed effects design was utilised to allow for the comparison of the stigmatism interventions. 80 participants were recruited opportunistically online and were provided with a text-based educational intervention combined with a counter-stereotypical exemplar video. This data was analysed using Bayesian Cumulative Probit Ordinal Regression Models.

There was an effect of condition which indicated that stigma inventory scores towards PWP were consistently higher pre-intervention, estimate $=0.44$, posterior probability $=.99$. No other estimates had $95 \%$ credible intervals (C.I) which did not include zero, making the results inconclusive. Despite this, the hypothesis distributions indicated that stigma inventory estimates tended towards the hypothesised results. The Perceived Social Distance model indicated that there may have been an effect of the intervention that was unique to the PWP condition although the C.I's for this hypothesis, and for all others, also included zero. The Social Distance model estimates did not produce any of the hypothesised results and none of the C.I's included zero.

Overall, this study found that a public stigmatism intervention has the potential to change stigmatising beliefs towards PWP and influence the perceived opinions of peers towards PWP. It also contests the proposed differences in PWP and alcoholism social distancing. Notably it provides a tentative first step in the development of a public stigmatism intervention which could potentially increase PWP participation in formal therapeutic services. 


\section{Introduction}

\section{Literature Review}

Paedophilic disorder is defined in the Diagnostic and Statistical Manual of Mental Health Disorders (DSM-V) as a sexual interest in prepubescent children. The diagnostic criteria specify that the individual must be a minimum of 16 years old and have a sexual interest in prepubescent children which causes fantasies that result in the abuse of a child 5 years younger than themselves or in severe distress for the individual (DSM-V). As such, paedophilic disorder does not require the individual to have acted on any fantasies to be diagnosed as having paedophilic disorder.

These non-offending paedophiles have only recently become the focus of psychological research. It was found, possibly as a result of media portrayal, the terms paedophile and child sex offender were thought to be, and treated as, interchangeable by the public - which is incorrect (Jahnke, Imhoff \& Hoyer, 2014). Around 51\% of the prison population of child sex offenders were considered paedophiles in the study by Seto (2008), which clearly indicates that paedophilia is neither necessary nor sufficient to cause a Child Sexual Abuse (CSA) offence. However, paedophilic disorder does constitute a high-risk factor in the perpetration of CSA and as such plays a role in the harm that it causes to the victims of it (Cantor \& McPhail, 2016).

CSA has many life-long negative effects for those who experience it, which are not isolated to severe mental health problems (Daray et al., 2016). It affects self-esteem; leads to sexual promiscuity; higher rates of unplanned pregnancies; increased likelihood of attempting suicide and even a lower gross personal income (Daray et al., 2016; Papalia, Ogloff, Cutajar \& Mullen, 2018). The effects of CSA can also result in the victim engaging in criminal activity. Papalia, Ogloff, Cutajar \& Mullen (2018), found that a sample of 2,759 CSA victims, compared with 2,677 community controls, had a higher average number of criminal offences and convictions, $23.6 \%$ CSA victims compared to $5.9 \%$ of controls.

This increased proportion of offences has been contested as being a result of the CSA and was postulated as being due to the victims being more exposed to: poverty; family disruption; poor parental adjustment; physical abuse; emotional deprivation and poor educational opportunities, which in turn creates a greater propensity for criminal involvement (Doidge, Higgins, Delfabbro \& Segal, 2017; Fergusson, Swain-Campbell \& Horwood, 2004; Malvaso, Delfabbro \& Day, 2017). However, when controlling for these demographic and developmental risk factors there is a clear and unique effect of CSA on the likelihood of criminal involvement which interacts with and possibly brings about the development of risky behaviours (Currie \& Tekin, 2012; Grasso et al., 2013; Lewis, 
McElroy, Harlaar \& Runyan, 2016; Malvaso, Delfabbro \& Day, 2017; Milaniak \& Widom, 2015; Walsh, Galea \& Koenen, 2012). Thus, the consequences of CSA for a victim, physically, mentally and behaviourally are ubiquitous and consistently negative.

Due to the widespread impact of CSA primary, secondary and tertiary prevention strategies have been implemented in the U.K. Primary prevention strategies aim to prevent harmful effects ever occurring, for example the education of children on how to recognise and avoid vulnerable situations. Tertiary prevention strategies are reactionary and include the application of therapeutic interventions for CSA offenders and victims (Brown \& Saied-Tessier, 2015; Fryda \& Hulme, 2014; Rheingold et al., 2014). Secondary prevention strategies for CSA utilise formal psychological therapeutic help for those at risk of committing an offence, such as non-offending paedophiles. Secondary prevention strategies have been available for a long time although their implementation has had little success and underwhelming participation (Levenson \& Grady, 2018). This failure in implementing secondary prevention strategies is particularly detrimental because it is the only approach which could directly deal with offenders, prior to any harm being caused. Furthermore, secondary prevention through formal therapy has the greatest propensity to reduce the financial burden of CSA.

The financial cost of CSA results from the combination of health implications, substance abuse, imprisonment and psychological therapy involved in most offences. The NHS and other governmental bodies deal with the victims, the offenders and the life-long consequences the offence results in (Saied-Tessier, 2014). Combined with the loss to the labour market Conti, Morris, Melnychuk \& Pizzo (2017) estimated a cost of $£ 89,390$ with a 95\% confidence interval of $£ 44,896$ 145,508 per victim, culminating in a net loss of at least $£ 1.6$ billion annually (Saied-Tessier, 2014). This lower estimate for the net cost was calculated when the number of incidences in the U.K was 22,573 , this has nearly tripled to 63,238 , therefore it can be inferred that the net loss has increased proportionally (Home Office, 2019; PSNI, 2018; Scottish Government 2018). The number of CSA offences has increased consistently every year and will turn into a greater drain on the economy unless something is done to reduce the number of victims and offenders. Thus, a secondary prevention strategy is uniquely beneficial, if implemented properly, as it can reduce both.

Secondary prevention strategies have been in place for a long time, through formal psychological help for non-offending paedophiles, with limited success and poor participation (Levenson \& Grady, 2018). Comparatively, informal anonymous help services are contacted by vastly higher numbers of non-offending paedophiles. For example, the 'Stop It Now!' organisation received 3,451 calls from this group in March 2015; constituting around half of the demand for the helpline (Horn et al., 
2015). From this, it can be inferred that there is a definite demand for these services and programs. However, there are factors preventing at-risk individuals seeking the formal psychological help that is currently available.

This has led to the consideration of what non-offending paedophiles experience when they do seek formal therapy (Levenson, Willis \& Vicencio, 2017). For instance, while help is technically available, it has been shown that $95 \%$ of psychotherapists, in Germany, are unwilling to work with this group, restricting the treatment options the individuals possess dramatically (Stiells-Glenn, 2010). Furthermore, of those who seek therapy, most find the experience to be unbeneficial as the therapist judges them harshly and does not view them as a whole person (Levenson \& Grady, 2018; Dymond \& Duff, 2020). In the event that an individual manages to find a therapist willing to help it is unlikely that they have a sufficient depth of knowledge on the disorder (Levenson \& Grady, 2018). Thus, some of the failure in implementing secondary prevention strategies is derived from the mental health professionals tasked with performing the intervention.

In response to this lack of considerate professional support, (Levenson \& Grady,2018; Stiells-Glenn, 2010), the main approach has been to address the stigmatising attitudes held by mental health professionals as reported by the clients throughout empirical studies (Jahnke \& Hoyer, 2013). In educational interventions for psychotherapists, there has been significant positive influence for the professionals involved, which reduced stigmatising beliefs and enhanced their motivation to work with non-offending paedophiles (Jahnke, Philipp \& Hoyer, 2015). Significant results in the adaption of stigmatising beliefs towards non-offending paedophiles, following an educational intervention regarding the controllability of the disorder, have also been found within psychology student populations (Imhoff \& Jahnke, 2017). Each of these three stigmatism studies were undertaken in Germany by: Imhoff \& Jahnke, (2017); Jahnke \& Hoyer, (2013); Jahnke, Philipp \& Hoyer, (2015). As a result of this research, Germany has successfully implemented secondary prevention strategies for CSA, in the form of the Dunkelfield Project (Beier et al., 2015). This initiative has received over 10,499 contacts, with 2,894 participants having attended their intervention programmes ("10,500 people asked for help", 2019). This reinforces the notion that challenging stigmatism could improve the effectiveness of secondary prevention strategies in the U.K, as utilised in the Dunkelfield Project (Beier et al., 2015).

Stigmatisation propagates factors such as isolation and loneliness that increase the risk individuals will offend (Cantor \& McPhail, 2016). Overall, the research indicates that stigmatisation of nonoffending paedophiles ultimately results in the offence they are incorrectly stigmatised for (Cantor \& McPhail, 2016). Stigmatism surrounding non-offending paedophiles in the U.K is postulated to have 
been constructed by media outlets, restricting the dissemination of expert opinion to the public, resulting in the conflation of the terms child sex offender and paedophile (Jahnke, Imhoff \& Hoyer, 2014; McCartan, 2004; McCartan, 2010). In most instances this stigmatism results in an attitude of intentionality towards the disorder to be taken by the public, (Jahnke, Imhoff \& Hoyer, 2014; Richards, 2017). This is opposed to the opinion of current research that defines paedophilia as a stable condition, uncontrolled in its occurrence, with urges that can be controlled by the individual (Cantor \& McPhail, 2016). It is essential that we explore stigma interventions for changing the publics opinion of non-offending paedophiles so that we can gain greater access to this group and prevent a greater cost, in harm and for the economy, (Grady, Levenson, Mesias, Kavanagh \& Charles, 2019; Parr \& Pearson, 2019).

\section{The Present Study}

The overall aim of this study is to: highlight the extent of public stigmatism towards non-offending paedophiles; assess the comparative effectiveness of a public stigmatism intervention for paedophilia, with a similarly stigmatised disorder, and provide insight on how a wide scale public stigmatism intervention may be implemented and received in the U.K. The secondary aim is the revalidation of the factor structure for the stigmatism scales used.

Research on stigmatism interventions highlight that exposing individuals to a vivid counterstereotypical exemplar has the greatest impact in the reduction of stigmatisation, when compared to a variety of other intervention methods (Lai et al., 2019). Meta analyses of stigmatism interventions, towards mental illness, highlight that educational interventions exhibit medium effect sizes reliably, (Morgan, Reavley, Ross, Too \& Jorm, 2018). Additionally, video-based interventions are more effective than face-to-face educational interventions (Janoušková et al., 2017). Furthermore, it has been shown that interventions which are of a narrative rather than professional style are more effective at both the explicit and implicit level for paedophilia (Harper, Bartels \& Hogue, 2016). Hence, an intervention which is of a narrative style, that incorporates a counter-stereotypical exemplar video of the stigmatised group, with additional educational information, could produce the best results in this study.

A similar structure of intervention was used to assess the malleability of stigmatising attitudes among training psychotherapists in Germany. However, this has not been tested on the public, or in the U.K, and the study itself lacked a sufficient number of participants (Jahnke, Phillip \& Hoyer, 2015). 
The present study is a conceptual replication of the study by Jahnke, Philip \& Hoyer (2015) and used a similar stigmatism intervention structure, with video material publicly available in English, as opposed to the original German videos used in the study. The primary difference is that the original study used a stigmatism intervention focussed on domestic violence as a control to compare with the non-offending paedophile intervention. The material for the domestic violence control is not available in the U.K, as such a comparison intervention towards alcohol use disorder (AUD) was used.

The choice of alcoholism for this research is based on the study by Feldmann \& Crandall, (2007), which found these two groups to have the highest level of social distance rating, and stigmatisation. Furthermore, there are scales which have been used to examine the stigmatising beliefs and social distance individuals have towards AUD and people with paedophilia (PWP) developed by Jahnke, Imhoff \& Hoyer (2014), acquired for this study from these authors. The paedophilia variant of the questionnaire was used in the original intervention study (Jahnke, Philip \& Hoyer, 2015). These scales were examined using an Exploratory Factor Analysis and Principal Component Analysis in the current paper to re-validate the factor structure, consisting of dangerousness; controllability; social distance and perceived social distance, for the English translation. However, these scales have been used by Jahnke, Imhoff and Hoyer (2014) to assess American opinions towards non-offending paedophiles.

A significant change in stigmatising beliefs towards non-offending paedophiles, following the intervention, is expected and that this will be greater than any change in the AUD group. There is also an expectation that there will be a higher average stigmatism score pre-intervention for paedophilia and a higher average stigmatism score for paedophilia post-intervention, when compared to the AUD group.

Hypothesis 1: There will be a decrease in stigmatising beliefs towards non-offending paedophiles following the intervention

Hypothesis 2: The decrease in stigmatising beliefs in the PWP group will be greater than any decrease in the AUD group

Hypothesis 3: The stigmatism scores pre-intervention will be significantly higher in the PWP condition

Hypothesis 4: The post-intervention stigmatism scores will be significantly higher for the PWP condition 


\section{Methodology}

\section{Participants}

A total of 80 participants were recruited, opportunistically, online using google forms and this allowed a wider array of individuals to be recruited from across the U.K. The only requirement was that the participants were residents in the U.K. The majority of responses were from Scottish residents. Additionally, a small portion of these individuals are students from the University of Glasgow.

The link that participants followed to the questionnaires was made using a google apps script and this randomly led them to either the PWP form or the AUD form. This served the function of randomly allocating participants to a condition.

The non-offending paedophile form received 39 responses ( 25 male, 13 female, 1 non-binary) and the AUD form received 41 responses ( 20 male, 20 female, 1 non-binary).

The distribution of age can be seen in Table 1. Levenes Tests for homogeneity of variance were insignificant between conditions, genders and genders within each condition.

\section{Table 1}

Summary of Age (years)

\begin{tabular}{lllll} 
Sex & condition & Mean Age & SD Age & IQR Age \\
\hline Female & AUD & 34.1 & 15.0 & 21.8 \\
Female & PWP & 33.3 & 13.2 & 17 \\
Male & AUD & 28.6 & 12.9 & 7.75 \\
Male & PWP & 38.5 & 17.1 & 32 \\
\hline
\end{tabular}

Note. Mean, standard deviation and inter-quartile range of age grouped by sex and condition.

\section{Materials}

For the assessment of stigmatising beliefs the Stigma Inventory (translated from German), Appendix A, Jahnke, Imhoff \& Hoyer (2014), Social Distance Scale (translated form German), Appendix B, Jahnke, Imhoff \& Hoyer (2014), and Perceived Social Distance Scale (translated from German), Appendix C, Jahnke, Schmidt, Geradt \& Hoyer (2015), were used and acquired with permissions from the authors of the scales. 
The questionnaires used have a reported:

$\alpha=.79$ for people who abuse alcohol, $\alpha=.89$ for people with paedophilia (PWP) for the Stigma Inventory Controllability factor, Appendix A (items 1 -3),

$\alpha=.90$ for people who abuse alcohol, $\alpha=.69$ for PWP for the Stigma Inventory Dangerousness factor, Appendix A (items 4-6),

$\alpha=.77$ for people who abuse alcohol, $\alpha=.82$ for PWP, for the Social Distance Scale, Appendix B, $\alpha=.84$ for the Perceived Social Distance Scale, Appendix C.

As mentioned, the interventions included an educational text on the topic of either Paedophilia, (Appendix D), or Alcoholism, Appendix E. This material was based on the information available on these subjects from the DSM-V and current professional opinion of the disorders themselves.

The videos used were "Inside The Life Of A 'Virtuous' Paedophile", Balson, Johnson, (producers) (2016), and "How I Overcame Alcoholism", Christian (2016). Both were shortened as both contained sections which were unbeneficial for the purpose of informative narrative. "Inside The Life Of $A$ 'Virtuous' Paedophile", (Balson .J, Johnson. N, (producers), 2016), contains information about the individual's fantasies and past abuse which we felt was not beneficial to the informative narrative. "How I Overcame Alcoholism", (Christian .C, 2016), contains a long section on a specific pharmaceutical intervention for alcoholism which did not add to the narrative and so this was removed, Appendix F.

\section{Design}

A $2 \times 2$ factorial design was used for this experiment to provide a comparison of the intervention effectiveness between the AUD and PWP conditions.

The data gathered was ordinal in nature, comprising of scores on a Likert scale from 1-6; strongly disagree to strongly agree. These scores constituted the Dependent Variable of this study.

As each participant was subjected to only one intervention topic, AUD or PWP, intervention topic was a between subjects Independent Variable. Both groups were exposed to topic specific intervention material and filled out the Stigma Inventory, Social Distance Scale and Perceived Social Distance Scale pre- and post-intervention, this produced repeated measures and so intervention was a within-subjects variable. 


\section{Procedure}

Participants followed a link which randomly led them to the AUD or non-offending paedophile form. A brief description of the experiment was provided, along with an online consent form before proceeding.

The participants were presented with the Stigma Inventory, Social Distance Scale and the Perceived Social Distance Scale. They were then given the educational text and viewed the relevant intervention video. Following the intervention material, the participants filled out the Stigma Inventory, Social Distance Scale and the Perceived Social Distance Scale again for comparison and to assess any change in beliefs.

Lastly, the participants were given a full debrief on the experiment (Appendix $\mathrm{G}$ ) and given the opportunity to comment on the experiment. Contact details were provided so they were able to contact the researcher for an update on the results of the experiment or in the event that they wished to revoke their data.

\section{Analysis}

\section{Primary Analyses.}

A Cumulative Probit Ordinal Regression Model was used to analyse the item responses. This was because the response data was ordinal and as such the psychological distance between the levels of the scale were unknown. Additionally, there was a possibility that this distance differed by participant, due to their interpretation of the scale. Ignoring these aspects of the data scale would have led to fundamentally flawed inference, Liddell \& Kruschke, (2018). Thus, an Ordinal Regression Model, whilst less common, was better suited for this analysis (Daykin \& Moffatt, 2002).

The specifying of the Cumulative model link was due to the assumption that each response categorized a level within a latent continuous variable. In this study responses categorized a level of stigmatism. The specification that this is a cumulative probit model indicates that the latent dependent variable, the concept of stigmatism, is normally distributed and continuous (Liu \& Agresti, 2005).

This model was implemented in R using the brm function from the BRMS package (Bürkner \& Vuorre, 2019). This function by default creates a fully Bayesian Framework for model estimation by using a Markov Chain Monte Carlo method to simulate the sampling from the distribution defined (McKinley, Morters \& Wood, 2015; Bürkner \& Vuorre, 2019). This provided a robust and extremely flexible method for analysing the ordinal data that was tailored to the present study. 
A separate Cumulative Probit Ordinal Regression Model was fitted for each Scale used, as each scale related to a single previously identified latent variable however there was no guarantee that this variable was the same for each scale.

Weakly informative priors were used as there was some information about the expected distribution in the literature, Appendix H, (Jahnke, Imhoff \& Hoyer ,2014; Jahnke, Schmidt, Geradt \& Hoyer, 2015). However, as this study was the first with a UK based sample it would have been unwise to use strongly informative priors, which aligns with the recommended practices of the research community (Depaoli \& van de Schoot, 2017; McElreath, 2016).

Four models were fitted and compared for each scale to assess the relative fit using the Leave One Out Cross Validation Information Criterion available in $\mathrm{R}$ in the loo package (Vehtari, Gelman \& Gabry ,2016). For the results of this testing and models assessed see Appendix $\mathrm{H}$.

\section{Secondary Analyses.}

For each condition, pre- and post-intervention, the internal consistency, reliability, of each scale was assessed using a bootstrapped Cronbachs Alpha, n = 10000 (Yuan, Guarnaccia \& Hayslip, 2003). An Exploratory Factor Analysis (EFA) and Principal Component Analysis (PCA) were also used to explore the structure of the questionnaires, this provided insight and allowed for the evaluation of the factor structure of each scale used.

\section{Results:}

\section{Descriptive Data Visualization:}

\section{Stigma Inventory Data.}

AUD Stigma Inventory Data. The Stigma Inventory Scores for AUD pre intervention had a mean of 16.5 , and a $20 \%$ trimmed mean of 15.9. This mean increased following the intervention to $17,20 \%$ trimmed mean increased to 16.9. Indicating an overall increase in stigmatising beliefs. In Figure 1, the distribution of stigma inventory results can be seen along with a noticeable increase in the interquartile-range (Pre $=13-18$, Post $=14-20)$. In figure 2 , the change in stigma inventory scores is represented and notably 19 of the 41 participants had higher stigma inventory scores postintervention 


\section{Figure 1}

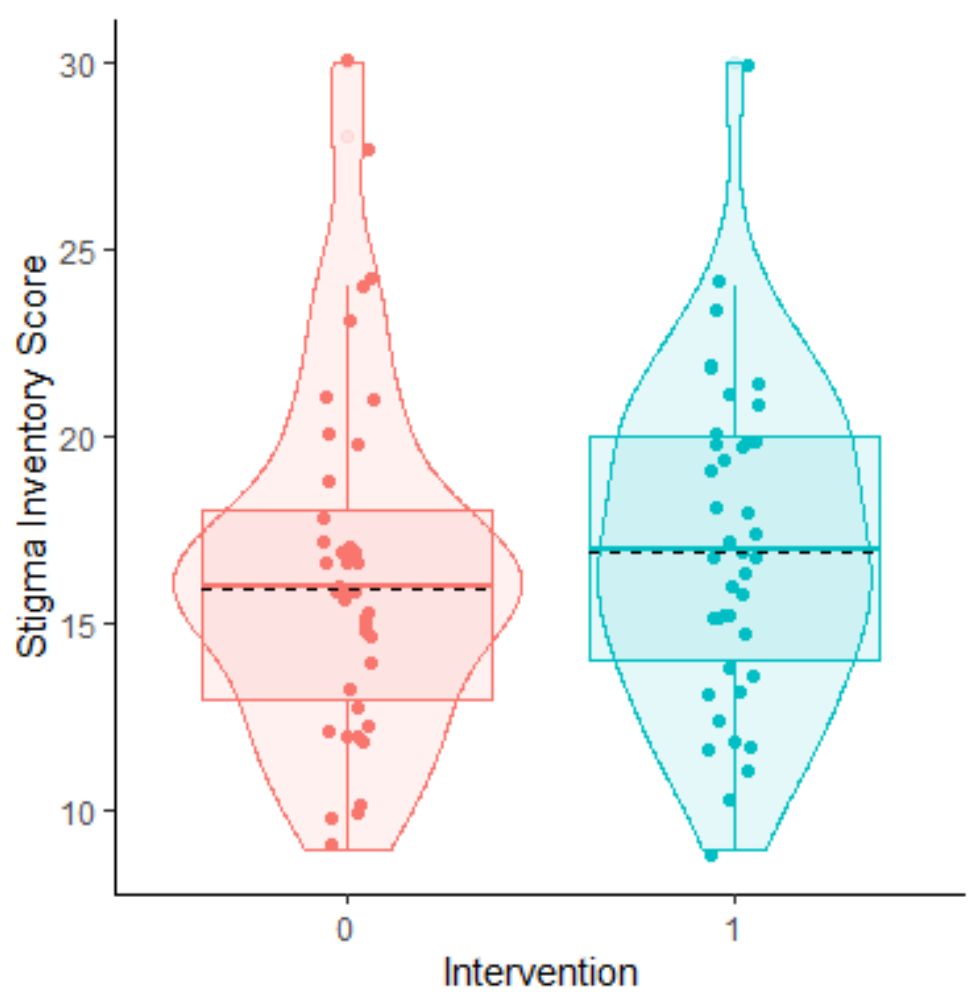

AUD Stigma Inventory Scores Pre and Post Intervention

Note. Intervention $0=$ pre-intervenion, Intervention $1=$ post-intervention. Dashed line represents $20 \%$ trimmed mean. 


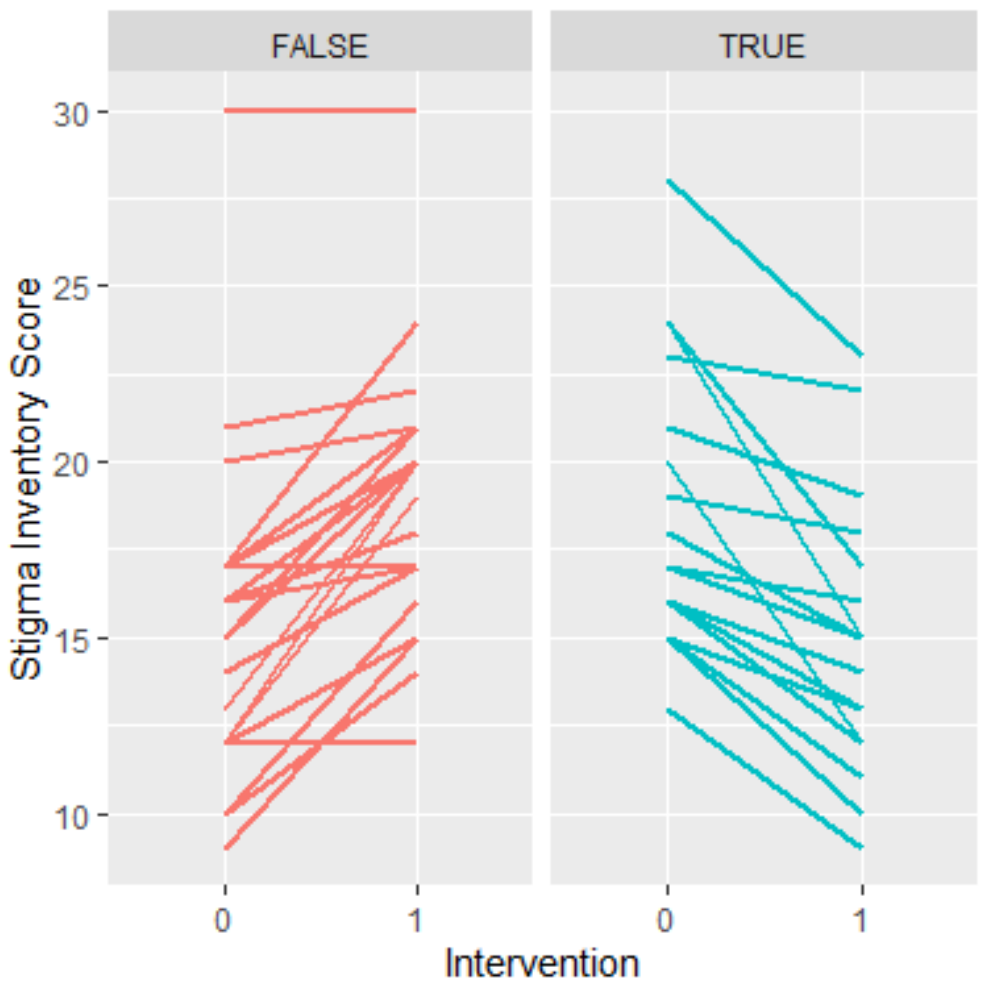

Figure 2 Intervention

AUD Participant-level changes in Stigma Inventory scores Pre- and Post-Intervention

Note. Each line represents one participant. TRUE indicates that the change in score was negative as expected, FALSE indicates that the change in score is positive which was not hypothesised.

PWP Stigma Inventory Data. The stigma inventory scores pre-intervention for the PWP condition had a higher mean than the AUD group (PWP mean $=20.1, A U D=16.5$ ), trimmed mean = 19.8. However, in the PWP condition the mean score decreased after the intervention (mean = 18.79), trimmed mean $=18.04$. The inter-quartile range increased following the intervention (pre = $16-24$, post $=14.5-24)$ and the standard deviation also increased (pre $=6.02$, post $=6.59$ ). These changes in variance are visualized in Figure 3 . The changes in stigma inventory scores are represented in Figure 4, notably scores increased following the intervention for 13 of the 39 participants, a smaller proportion of scores than in the AUD condition. 


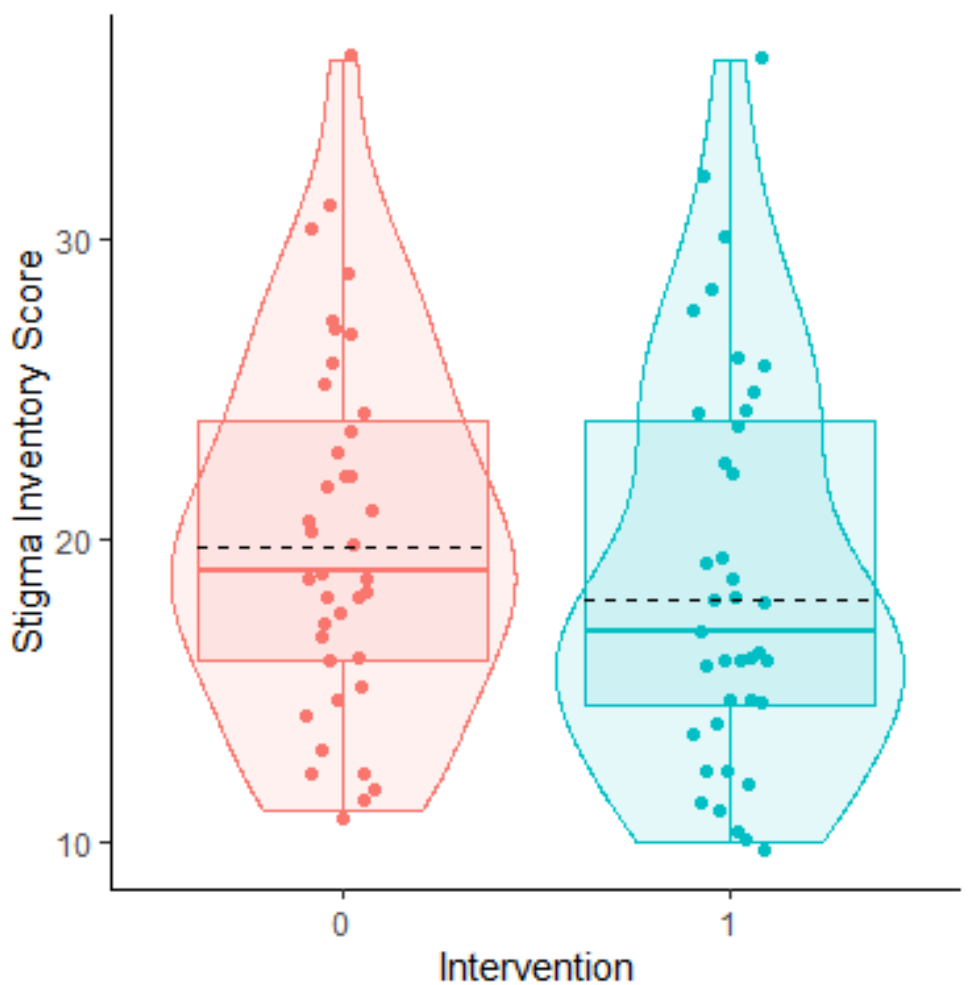

Figure 3

PWP Stigma Inventory Scores Pre and Post Intervention

Note. Intervention $0=$ pre-intervenion, Intervention $1=$ post-intervention. Dashed line represents $20 \%$ trimmed mean. 


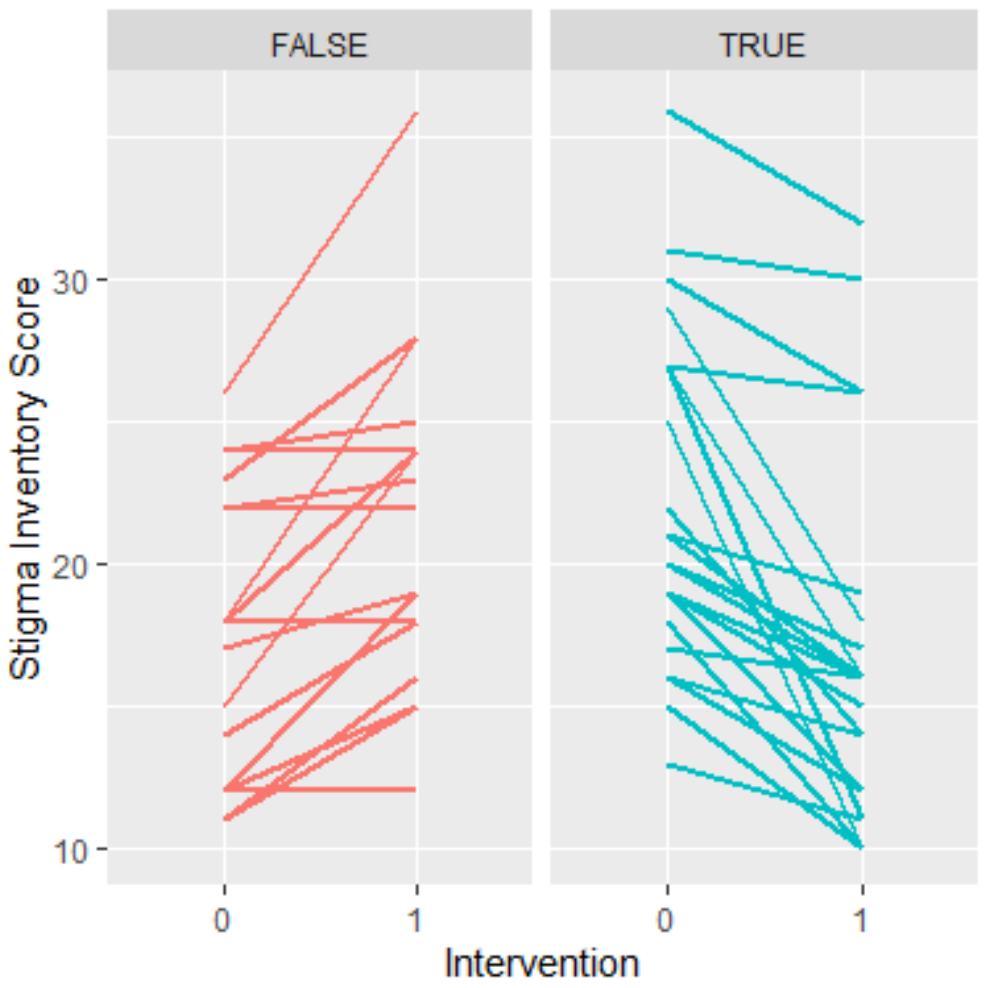

Figure 4 Intervention

PWP Participant-level changes in Stigma Inventory scores Pre- and Post-Intervention

Note. Each line represents one participant. TRUE indicates that the change in score was negative as expected, FALSE indicates that the change in score is positive which was not hypothesised.

\section{Social Distance Scale Data}

AUD Social Distance Scale Data. In the AUD condition the mean scores for Social Distance increased following the intervention, (mean Pre $=22.41$, mean Post $=23.61)$, trimmed mean Pre $=$ 22.88, Post $=23.92$. The standard deviation also decreased (Pre $=4.71$, Post $=4.30$ ) along with the inter-quartile range (Pre $=19-26$, Post $=21-27$ ) indicating an overall reduction in the variance of scores, visualized in Figure 5. In Figure 6, the changes in social distance scores can be observed and unexpectedly 23 of the 41 participants scores increased following the intervention. 


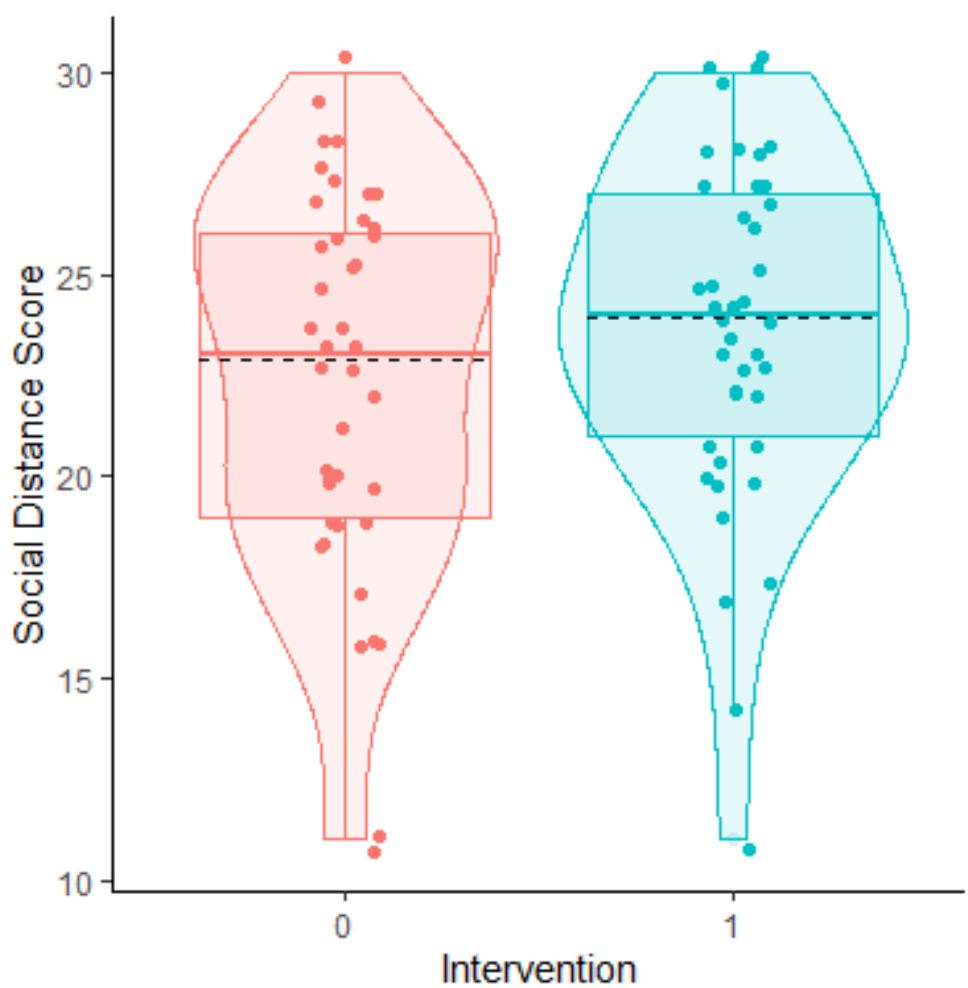

Figure 5

AUD Social Distance Scores Pre and Post Intervention

Note. Intervention $0=$ pre-intervenion, Intervention $1=$ post-intervention. Dashed line represents $20 \%$ trimmed mean. 


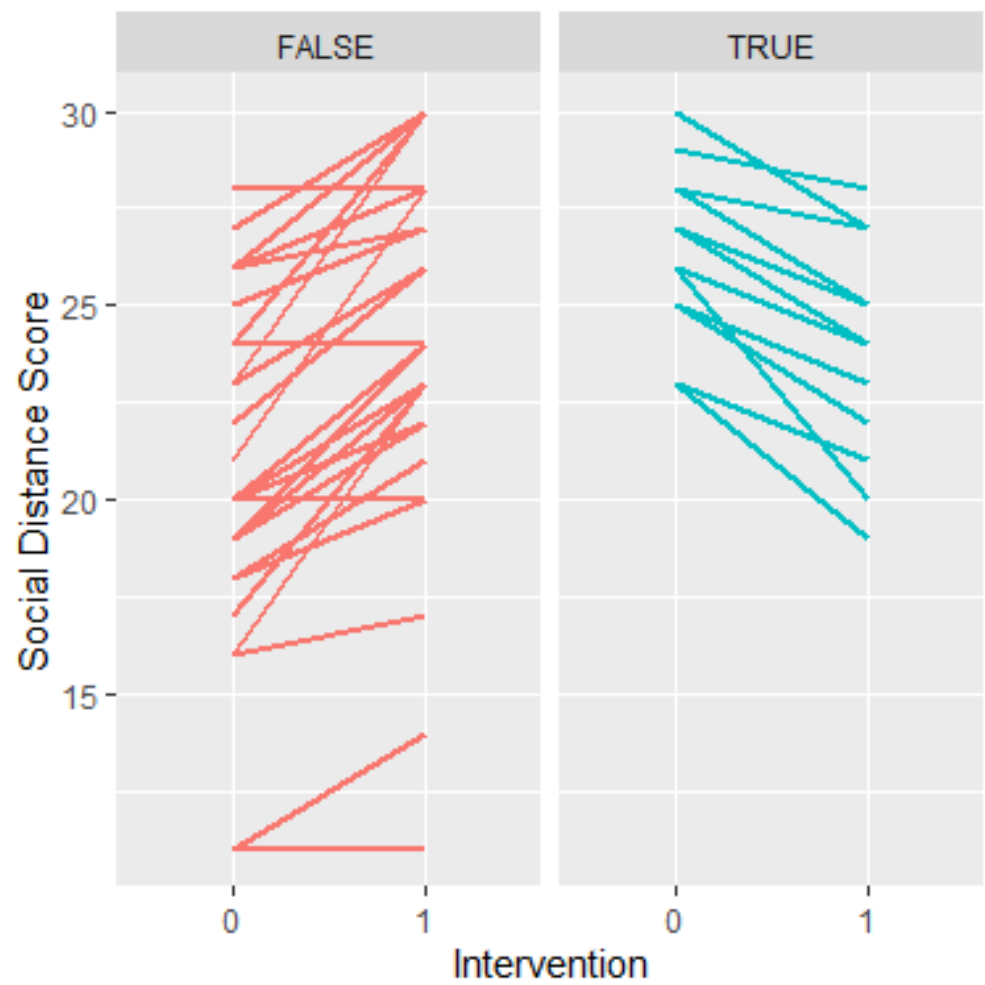

Figure 6

AUD Participant-level changes in Perceived Social Distance scores Pre-and Post-Intervention

Note. Each line represents one participant. TRUE indicates that the change in score was negative as expected, FALSE indicates that the change in score is positive which was not hypothesised.

PWP Social Distance Scale Data. In the PWP group the Pre-Intervention mean was higher than for the AUD group (mean Pre $=24.66$, trimmed mean $=23.9$ ) and this increased following the intervention (mean Post $=25.94$, trimmed mean $=26.48$ ). As was observed in the AUD group the inter-quartile range increased (Pre $=20-28$, Post $=23-30$ ) in PWP condition however contrary to the AUD group the standard deviation also increased (Pre $=6.38$, Post $=7.07$ ) indicating that the variance in scores may have increased following the intervention, Figure 7. The changes in Social Distance scores can be seen in Figure 8 and 23 of the 39 participants scores increased following the intervention, which is similar to the division of changes seen in the AUD condition. 


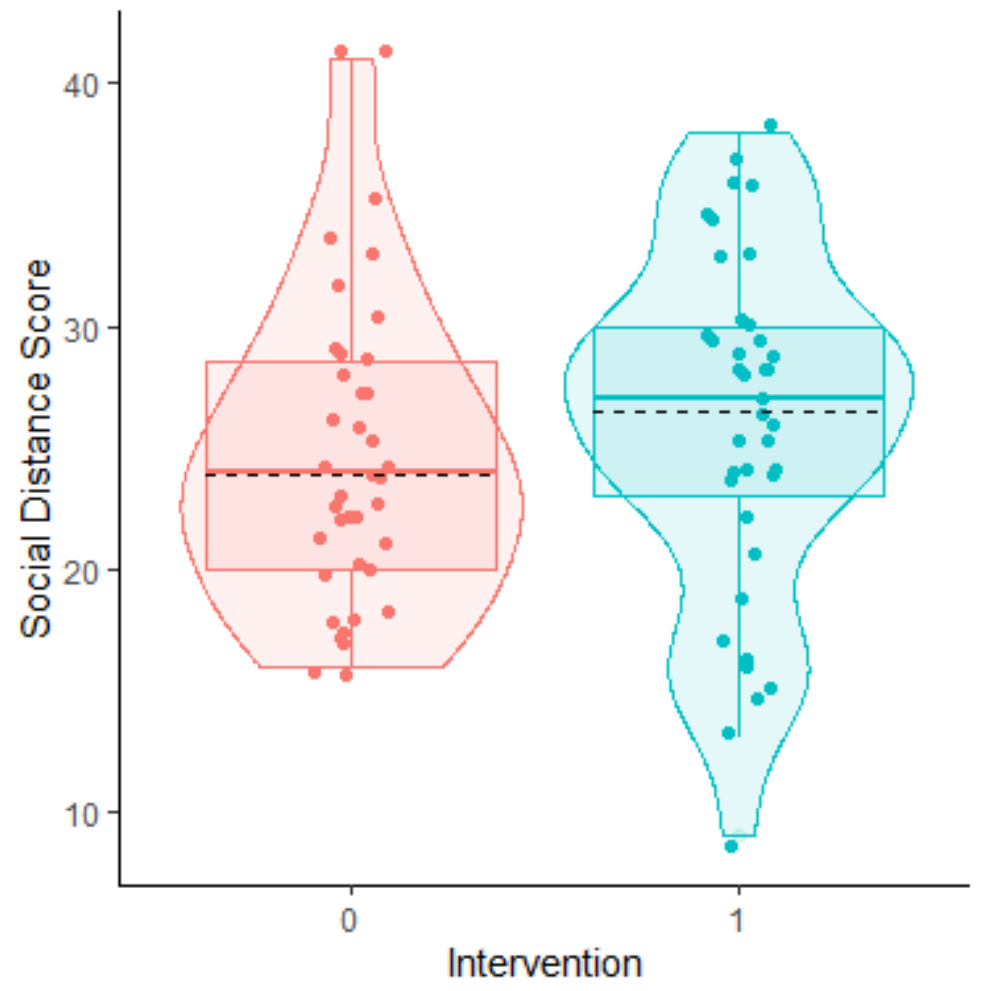

Figure 7

PWP Social Distance Scores Pre and Post Intervention

Note. Intervention $0=$ pre-intervenion, Intervention $1=$ post-intervention. Dashed line represents $20 \%$ trimmed mean. 


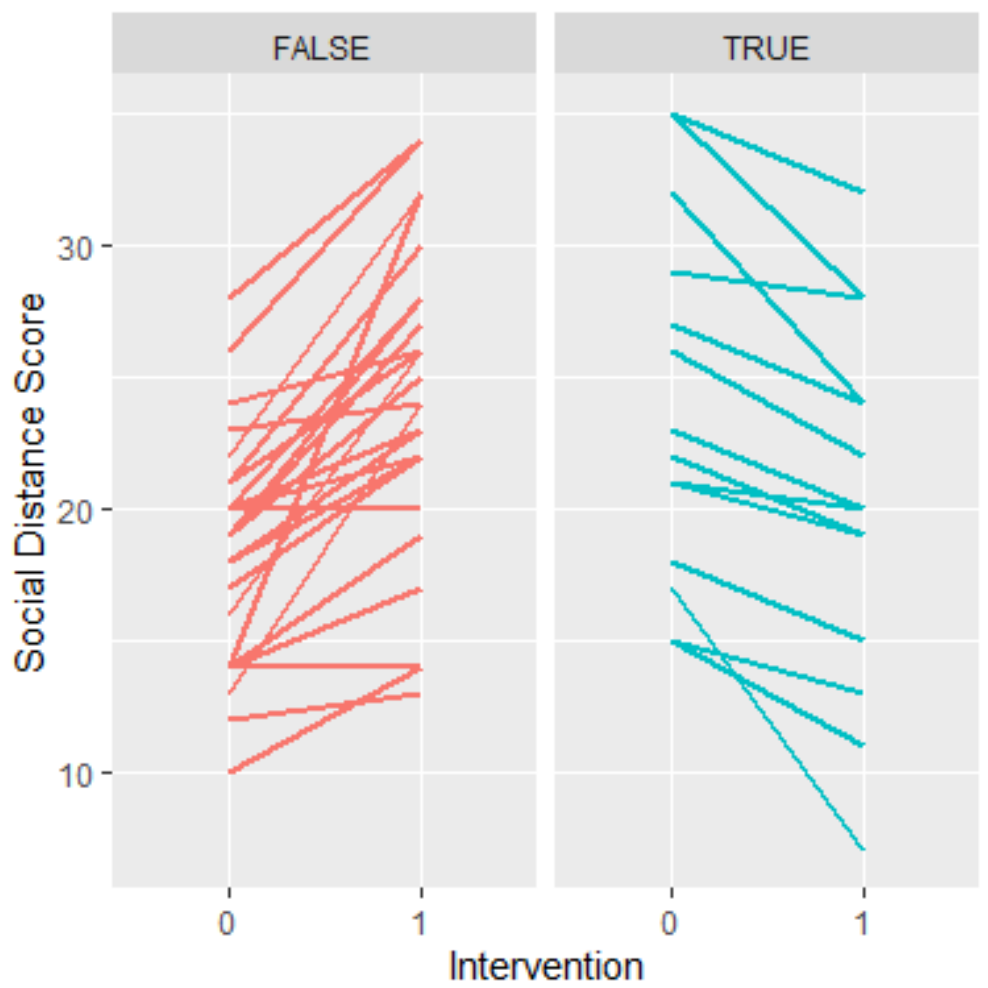

Figure 8

PWP Participant-level changes in Social Distance scores Pre- and Post-Intervention

Note. Each line represents one participant. TRUE indicates that the change in score was negative as expected, FALSE indicates that the change in score is positive which was not hypothesised.

\section{Perceived Social Distance Data}

AUD Perceived Social Distance Data. The mean AUD Perceived Social Distance score increased following the intervention (Pre $=21.02$, Post $=21.6)$, trimmed mean (Pre $=20.56$, Post $=$ 21.24). The standard deviation also increased (Pre $=4.22$, Post $=4.32$ ) and the inter-quartile range increased (Pre=18-23, Post=19-25) indicating an increased variance, Figure 9.18 of the 41 participants Perceived Social Distance scores increased following the intervention, these changes are seen in Figure 10. 


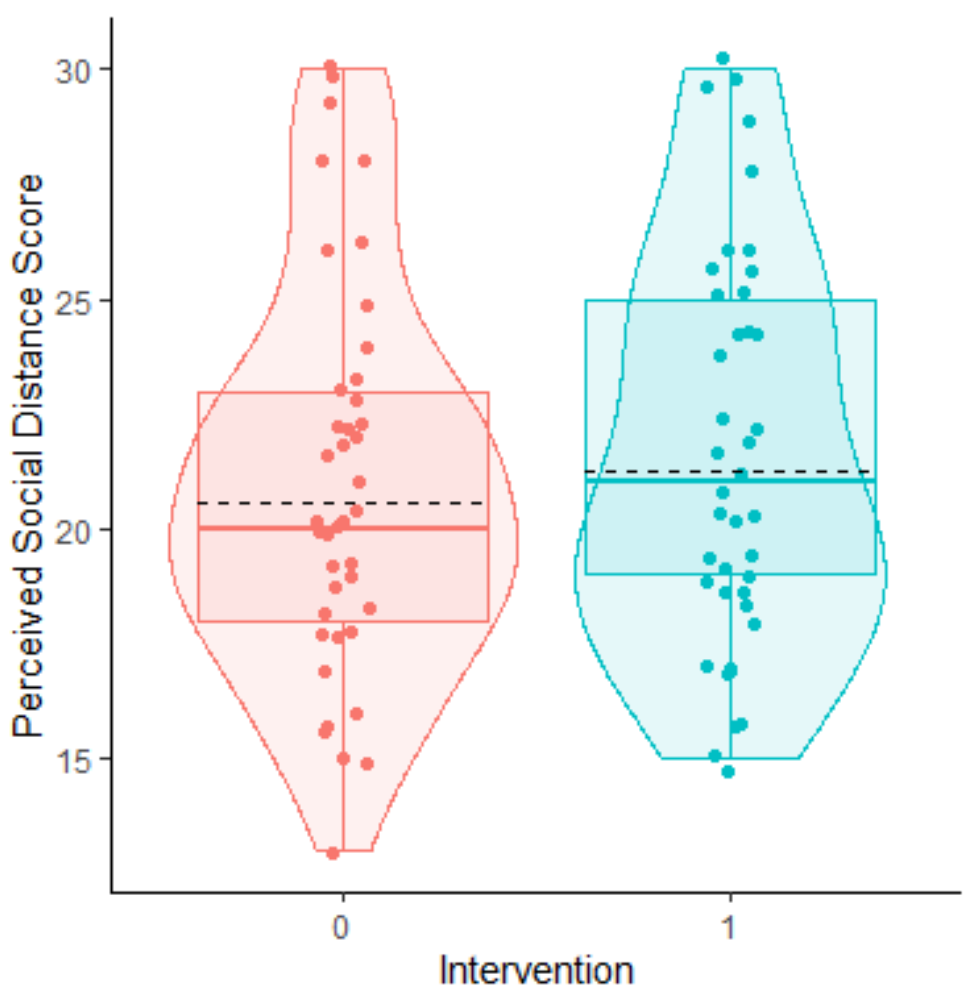

Figure 9

AUD Perceived Social Distance Scores Pre and Post Intervention

Note. Intervention 0 = pre-intervenion, Intervention $1=$ post-intervention. Dashed line represents $20 \%$ trimmed mean. 


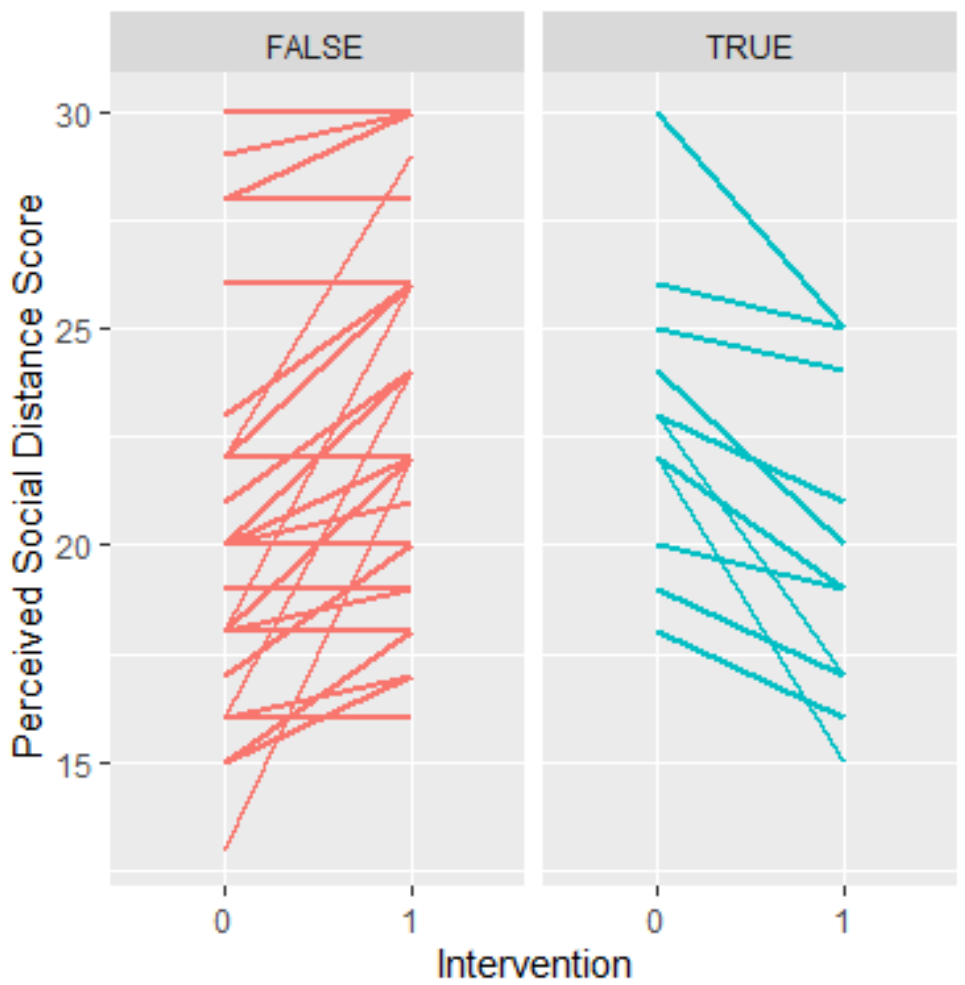

Figure 10

AUD Participant-level changes in Perceived Social Distance scores Pre-and Post-Intervention

Note. Each line represents one participant. TRUE indicates that the change in score was negative as expected, FALSE indicates that the change in score is positive which was not hypothesised.

PWP Perceived Social Distance Data. In contrast the PWP Perceived Social Distance mean decreased $($ Pre $=17.51$, Post $=17.15)$, trimmed mean $($ Pre $=17.52$, Post $=17.31)$. The standard deviation increased $($ Pre $=3.72$, Post $=3.89)$ along with the inter-quartile range $($ Pre $=15-20$, Post $=$ 14-20), Figure 11 illustrates this change in variance. Changes in perceived social distance were positive for 10 of he 39 participants, Figure 12, which is a smaller proportion than in the AUD condition. 


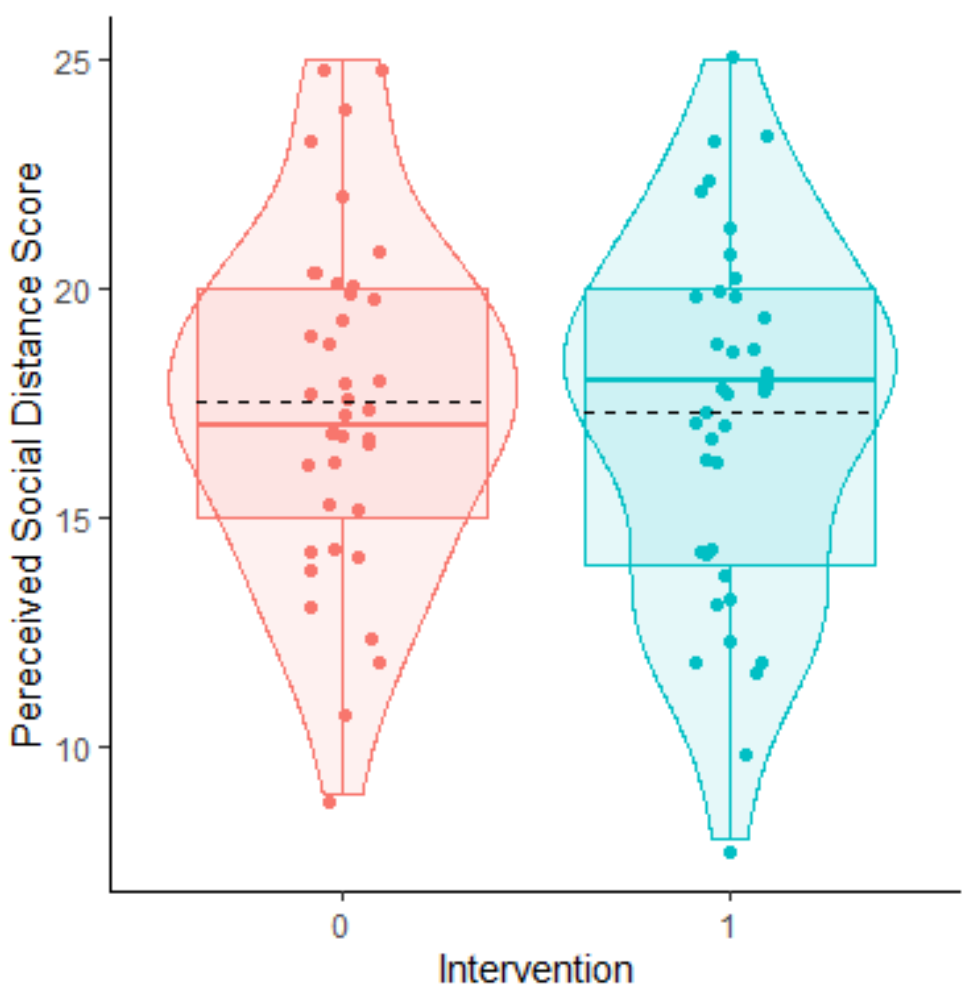

Figure 11

PWP Perceived Social Distance Scores Pre and Post Intervention

Note. Intervention $0=$ pre-intervenion, Intervention $1=$ post-intervention. Dashed line represents $20 \%$ trimmed mean. 


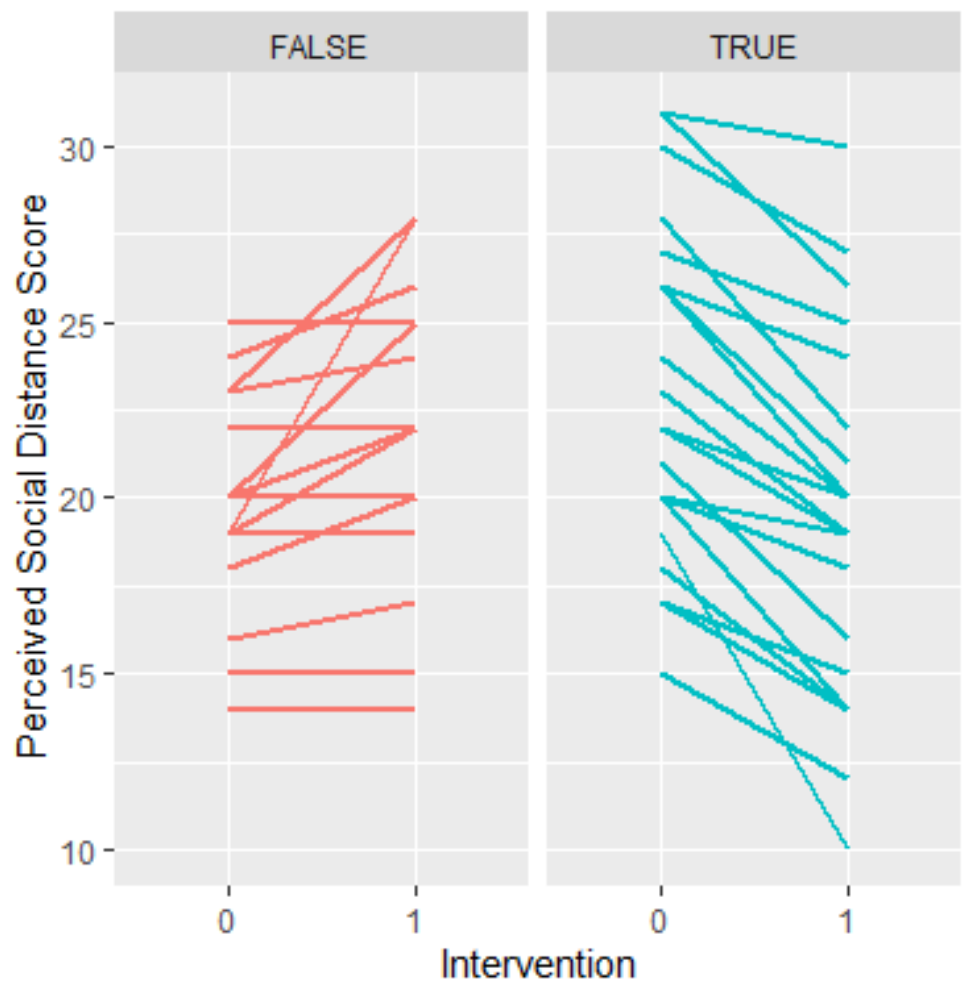

Figure 12

PWP Participant-level changes in Perceived Social Distance scores Pre-and Post-Intervention

Note. Each line represents one participant. TRUE indicates that the change in score was negative as expected, FALSE indicates that the change in score is positive which was not hypothesised.

\section{Bayesian Ordinal Regression Model Results}

\section{Model Selection Details}

All selected models have tables of all estimates calculated in Appendix I. Each scale has four associated models with varying formulas, this was done to compare the fits of each potential model to the data. All models were fitted with weakly informative priors, except model 1 used noninformative priors, this is the case for each scale. This was required for the comparison of the effect of the priors on the models fitted. Tables of the results of the LOOIC values can be found in Appendix $H$. These values were used to decide which model fitted the data optimally. The models chosen were: Stigma Inventory Model 3, Social Distance Scale Model 4 and Perceived Social Distance Model 4. No group level correlations produced consistent significant effects in any of the models, for brevity they are not reported in the following results (Appendix I). Results for coefficients are reported: coefficient name, estimate [95\% credible interval]. Results of general non-linear 
hypothesis tests are reported: Hypothesis $\mathrm{N}$ (Hypothesis formula) = estimate [95\% credible interval] $=$ posterior probability.

\section{Stigma Inventory Model Results}

The group-level effects for participants, Table 2, indicated that the effect of the intervention varied greatly dependent on the participant. The group-level effects for items, Table 3, highlights the differing pattern of item responses between the conditions. This suggests that the ratings for each item was dependent on the condition the individual was in.

Table 2

Stigma Inventory Bayesian Ordinal Regression Group-Level Effects for Participants

\begin{tabular}{lccccr} 
& Estimate & Est.Error & $\mathrm{I}-95 \% \mathrm{Cl}$ & $\mathrm{u}-95 \% \mathrm{Cl}$ & Rhat \\
\hline $\mathrm{sd}$ (Intercept) & 0.54 & 0.07 & 0.40 & 0.69 & 1.00 \\
$\mathrm{sd}($ Intervention1) & 0.26 & 0.15 & 0.02 & 0.54 & 1.00 \\
& & & & & \\
\hline
\end{tabular}

Note. Number of levels: 80 . Rhat refers to the brms measure of model convergence and should = 1.00 .

Table 3

Stigma Inventory Bayesian Ordinal Regression Group-Level Effects for Items

\begin{tabular}{lccccc} 
& Estimate & Est.Error & $\mathrm{I}-95 \% \mathrm{Cl}$ & $\mathrm{u}-95 \% \mathrm{Cl}$ & Rhat \\
\hline $\mathrm{sd}$ (Intercept) & 0.06 & 0.06 & 0.00 & 0.21 & 1.00 \\
$\mathrm{sd}($ condition1) & 0.21 & 0.14 & 0.02 & 0.55 & 1.00 \\
& & & & & \\
\hline
\end{tabular}

Note. Number of levels: 6

The population-level effects for the Stigma Inventory scale, Table 4, indicated that there was an effect of condition $1,0.44[0.08,0.80]$. This indicated that pre-intervention the stigma scores for the PWP condition were higher. This is supported by the results for Hypothesis 3 (condition1 estimate > $0)=0.44[0.13,0.71]=.99$. 
The estimate for intervention $1,0.09[-0.12,0.28]$, is unexpected as the estimate is low but also positive, however the credible interval (C.I) indicated that the intervention did not consistently increase stigmatism ratings, and in instances may have decreased it.

The interaction estimate condition 1 :Intervention1, $-0.21[-0.49,0.08]$, indicated that there was a moderate negative effect of the intervention which was dependent on the condition. Combined with the Intervention 1 estimate, this interaction suggests that generally there was a decrease in stigmatism following the intervention for the PWP condition, supporting hypothesis 1 , and that this was greater than the decrease associated with the AUD condition, supporting hypothesis 2 .

\section{Table 4}

Stigma Inventory Bayesian Ordinal Regression Population Level Effects

\begin{tabular}{lccccc} 
& Estimate & Est.Error & $\mathrm{l}-95 \% \mathrm{Cl}$ & $\mathrm{u}-95 \% \mathrm{Cl}$ & Rhat \\
\hline Intercept[1] & -0.74 & 0.12 & -0.98 & -0.51 & 1.00 \\
Intercept[2] & -0.08 & 0.12 & -0.30 & 0.15 & 1.00 \\
Intercept[3] & 0.61 & 0.12 & 0.37 & 0.83 & 1.00 \\
Intercept[4] & 1.18 & 0.12 & 0.94 & 1.42 & 1.00 \\
Intercept[5] & 1.86 & 0.13 & 1.60 & 2.11 & 1.00 \\
condition1 & 0.44 & 0.18 & 0.08 & 0.80 & 1.00 \\
Intervention1 & 0.09 & 0.10 & -0.12 & 0.28 & 1.00 \\
condition1:Intervention1 & -0.21 & 0.15 & -0.49 & 0.08 & 1.00 \\
\hline
\end{tabular}

Note. Condition dummy coding ( $\mathrm{AUD}=0, \mathrm{PWP}=1)$, Intervention dummy coding (Pre $=0$, Post $=1$ )

For Hypothesis 1 (condition1 + Intervention $1+$ condition1:Intervention $1<$ condition 1$)=-0.12[-$ $0.30,0.06]=.87$. This indicated that there was generally a decrease in the stigmatism scores towards PWP post-intervention, however, this effect was not consistent.

For Hypothesis $2(($ Intervention $1+$ condition1:Intevention 1$)<($ Intervention1 $))=-0.21[-0.44,0.03]$ $=.92$. This suggested that in most instances the decrease in stigmatism scores towards PWP was greater than the decrease in stigmatism towards AUD.

For hypothesis $4(($ condition $1+$ Intervention $1+$ condition $1:$ Intervention 1$)>($ Intervention 1$))=0.23[-$ $0.08,0.54]=.90$. This indicates that stigmatism scores were generally higher for the PWP group post intervention compared to the stigmatism towards the AUD group post-intervention. However, it 
should be noted that this difference is no longer large enough to fully distinguish the groups, as was the case pre-intervention.

\section{Social Distance Scale Model Results}

The group-level effects for participants, Table 5, indicated that there was a notable variance in the effect of the intervention that was dependent on the participant. The group-level effects for items indicated that item response patterns varied consistently between conditions, between interventions and between interventions within the PWP condition, Table 6.

Table 5

Social Distance Scale Bayesian Ordinal Regression Group-Level Effects for Participants

\begin{tabular}{lccccc} 
& Estimate & Est.Error & $\mathrm{I}-95 \% \mathrm{Cl}$ & $\mathrm{u}-95 \% \mathrm{Cl}$ & Rhat \\
\hline $\mathrm{sd}$ (Intercept) & 0.58 & 0.07 & 0.44 & 0.73 & 1.00 \\
$\mathrm{sd}($ Intervention1) & 0.20 & 0.12 & 0.01 & 0.44 & 1.00 \\
\hline
\end{tabular}

Note. Number of levels:80

Table 6

Social Distance Scale Bayesian Ordinal Regression Group-Level Effects for Items

\begin{tabular}{lccccc} 
& Estimate & Est.Error & $\mathrm{l}-95 \% \mathrm{Cl}$ & $\mathrm{u}-95 \% \mathrm{Cl}$ & $\mathrm{Rhat}$ \\
\hline $\mathrm{sd}$ (Intercept) & 0.61 & 0.20 & 0.34 & 1.11 & 1.00 \\
$\mathrm{sd}($ condition1) & 0.42 & 0.19 & 0.15 & 0.88 & 1.00 \\
$\mathrm{sd}($ Intervention1) & 0.41 & 0.20 & 0.11 & 0.90 & 1.00 \\
sd(condition1:Intervention1) & 0.79 & 0.29 & 0.39 & 1.47 & 1.00 \\
\hline
\end{tabular}

Note. Number of levels: 6

The Social Distance scale population-level estimates, Table 7, for condition1, $-0.26[-0.70,0.21]$ indicated that the Social Distance scores were generally lower for the PWP condition preintervention. However, the estimated error, 0.24, was relatively large and the C.I includes zero. Therefore, the effect of condition in this instance is inconclusive. Hypothesis 3 (condition1 $>0$ ) $=-$ $0.26[-0.63,0.13]=.13$, indicates that the majority of PWP scores were not higher than the AUD group pre-intervention as hypothesised. 
The Intervention 1 effect, $0.19[-0.17,0.54]$ indicates that Social Distance scores generally increased for both conditions however, the estimated error $(0.18)$ is relatively high compared to the estimate and the C.I includes zero and so these results are inconclusive.

The interaction effect condition1:Intervention $1=0.08[-0.59,0.90]$ had a large C.I which included zero as such these results are inconclusive.

\section{Table 7}

Social Distance Scale Bayesian Ordinal Regression Population-Level Effects

\begin{tabular}{lccccc} 
& Estimate & Est.Error & I-95\% Cl & $\mathrm{u}-95 \% \mathrm{Cl}$ & Rhat \\
\hline Intercept[1] & -1.55 & 0.27 & -2.08 & -0.97 & 1.00 \\
Intercept[2] & -0.85 & 0.27 & -1.37 & -0.28 & 1.00 \\
Intercept[3] & -0.12 & 0.27 & -0.64 & 0.44 & 1.00 \\
Intercept[4] & 0.45 & 0.27 & -0.07 & 1.00 & 1.00 \\
Intercept[5] & 1.04 & 0.27 & 0.53 & 1.61 & 1.00 \\
condition1 & -0.26 & 0.24 & -0.70 & 0.21 & 1.00 \\
Intervention1 & 0.19 & 0.18 & -0.17 & 0.54 & 1.00 \\
condition1:Intervention1 & 0.08 & 0.28 & -0.49 & 0.64 & 1.00 \\
\hline
\end{tabular}

Note. Condition dummy coding ( $\mathrm{AUD}=0, \mathrm{PWP}=1)$, Intervention dummy coding (Pre $=0$, Post $=1$ )

For Hypothesis 1 (condition $1+$ Intervention $1+$ condition1: Intervention $1<$ condition 1$)=.27[-0.19$, $0.71]=.15$. This indicates that the intervention generally increased Social Distance scores in most instances. However, this result is inconclusive as the C.I includes zero.

For Hypothesis $2(($ Intervention $1+$ condition1:Intevention 1$)<($ Intervention 1$))=0.08[-0.39,0.54]=$ .4 . This suggests that the effect of the intervention was not significantly different between conditions.

For hypothesis $4(($ condition $1+$ Intervention $1+$ condition $1:$ Intervention 1$)>($ Intervention 1$))=0.18[-$ $0.79,0.44]=.30$. These results indicate that the majority of scores for Social Distance for the PWP condition post-intervention were not higher than the Social Distance Scores for the AUD condition. Therefore, the differences between the groups was insubstantial or the true difference is lower, the opposite of what was hypothesised. 


\section{Perceived Social Distance Scale}

The group-level effects for the participants indicated that there was some degree of variance in the effect of the intervention, however, the C.I includes zero and so this estimated variance is inconclusive, Table 8. The group-level effects for the items indicated that there was a notable amount of variance in item response patterns between conditions, Table 9. However, whilst the item response patterns were shown to vary between interventions and between interventions within the PWP condition both estimates had lower 95\% C.I values close to zero and so these effects should be interpreted with scrutiny.

\section{Table 8}

Perceived Social Distance Scale Bayesian Ordinal Regression Group-Level Effects for Participants

\begin{tabular}{lccccc} 
& Estimate & Est.Error & $\mathrm{I}-95 \% \mathrm{Cl}$ & $\mathrm{u}-95 \% \mathrm{Cl}$ & Rhat \\
\hline $\mathrm{sd}$ (Intercept) & 0.39 & 0.06 & 0.28 & 0.51 & 1.00 \\
$\mathrm{sd}$ (Intervention1) & 0.08 & 0.06 & 0.00 & 0.22 & 1.00 \\
\hline
\end{tabular}

Note. Number of levels:80

Table 9

Perceived Social Distance Scale Bayesian Ordinal Regression Group-Level Effects for Items

Estimate Est.Error $\quad 1-95 \% \mathrm{Cl} \quad$ u-95\% Cl $\quad$ Rhat

\begin{tabular}{llllll}
$\mathrm{sd}($ Intercept) & 0.77 & 0.26 & 0.43 & 1.39 & 1.00 \\
$\mathrm{sd}($ condition1) & 0.90 & 0.30 & 0.50 & 1.64 & 1.00 \\
sd(Intervention1) & 0.21 & 0.16 & 0.01 & 0.60 & 1.00 \\
sd(condition1:Intervention1) & 0.35 & 0.24 & 0.02 & 0.91 & 1.00 \\
\hline
\end{tabular}

Note. Number of levels: 6

The condition1 estimate for the Perceived Social Distance Scale $0.09[-0.66,0.83]$. The C.I indicated that the estimate was not consistently above zero and, therefore, it could not be concluded that the PWP condition score for Perceived Social Distance was higher in $95 \%$ of instances. Hypothesis 3 (condition $1>0)=0.09[-0.51,0.7]=.61$, indicated that Perceived Social Distance scores were not consistently higher than the AUD condition pre-intervention. 
The Intervention1 estimate for the Perceived Social Distance scale $0.08[-0.20,0.34]$ indicated that the intervention had a varying, inconsistent effect on Perceived Social Distance scores.

The interaction estimate condition 1 :Intervention $1-0.19[-0.62,0.22]$ indicated that the intervention generally reduced stigmatism scores in the PWP condition. However, the estimated error, 0.19 , was equal to the effect estimate and the C.I included zero. As such, the possible unique effect of the intervention on the PWP condition cannot be discerned conclusively.

Table 10

Perceived Social Distance Scale Bayesian Ordinal Regression Population-level Effects

\begin{tabular}{lccccc} 
& Estimate & Est.Error & $\mathrm{I}-95 \% \mathrm{Cl}$ & $\mathrm{u}-95 \% \mathrm{Cl}$ & Rhat \\
\hline Intercept[1] & -1.29 & 0.32 & -1.94 & -0.65 & 1.00 \\
Intercept[2] & -0.47 & 0.32 & -1.11 & 0.16 & 1.00 \\
Intercept[3] & 0.05 & 0.32 & -0.59 & 0.68 & 1.00 \\
Intercept[4] & 0.47 & 0.32 & -0.18 & 1.11 & 1.00 \\
Intercept[5] & 1.36 & 0.32 & 0.71 & 2.01 & 1.00 \\
condition1 & 0.09 & 0.37 & -0.66 & 0.83 & 1.00 \\
Intervention1 & 0.08 & 0.13 & -0.20 & 0.34 & 1.00 \\
condition1:Intervention1 & -0.19 & 0.19 & -0.56 & 0.21 & 1.00 \\
\hline
\end{tabular}

For Hypothesis 1 (condition $1+$ Intervention $1+$ condition $1:$ Intervention $1<$ condition 1$)=-0.11[-$ $0.42,0.21]=.75$. This suggests that most estimates indicated a decrease in the Perceived Social Distance scale for the PWP condition following the intervention. However, as the credible interval includes zero this decrease was not consistent therefore the true effect of this cannot be concluded.

For Hypothesis $2(($ Intervention $1+$ condition1:Intevention 1$)<($ Intervention 1$))=-0.19[-0.49,0.13]=$ .85. These results indicated that the intervention was generally more effective for the PWP condition than the AUD condition. However, as the C.I included zero the purported increased efficacy of the intervention for the PWP condition cannot be fully discerned.

For Hypothesis $4(($ condition $1+$ Intervention $1+$ condition $1:$ Intervention 1$)>($ Intervention 1$))=-0.09$ $[-0.73,0.57]=.39$. This suggested that either no difference between conditions for the Perceived Social Distance scores post intervention was present or that the PWP scores were mostly lower than the AUD condition, neither can be confirmed by these results. 


\section{Questionnaire Analysis}

\section{Bootstrapped Alpha}

The controllability aspect of the Stigma Inventory for the PWP, $\alpha=.783[.560, .902]$, and AUD, $\alpha=$ $.714[.420, .860]$, conditions had an acceptable reliability mean $\alpha>0.7$, Table 11 . However, the dangerousness aspect of the Stigma Inventory was not as reliable with the AUD condition being borderline acceptable, $\alpha=.698[.356, .865]$, and the PWP, $\alpha=.560[.124, .773]$ condition failing to meet the $\alpha>0.7$ threshold.

The Social Distance Scale mean bootstrapped alpha for the PWP condition, $\alpha=.674[.421, .801]$, was marginally below the threshold of $\alpha>0.7$. However, the AUD bootstrapped mean alpha estimate, $\alpha$ $=.599[.328, .737]$, was clearly under this threshold, Table 11 .

The Perceived Social Distance Scale mean bootstrapped alphas for the PWP and AUD condition were below the $\alpha>0.7$ threshold and were the lowest values of any scale used in this study, Table 11 . 


\section{Table 11}

Bootstrapped Alpha values for Scales and Conditions

\begin{tabular}{|c|c|c|c|c|c|c|c|}
\hline Condition & Scale & Pre $\alpha$ & Pre C.I $\alpha$ & Post $\alpha$ & Post C.I $\alpha$ & Mean $\alpha$ & Mean C.I $\alpha$ \\
\hline$A \cup D$ & Control & .676 & $.370-0.835$ & .752 & $.469-.880$ & .714 & $.420-.860$ \\
\hline$P W P$ & Control & .691 & $.386-.852$ & .876 & $.733-.953$ & .783 & $.560-.902$ \\
\hline$A U D$ & Danger & .759 & $.500-.887$ & .638 & $.213-.842$ & .698 & $.356-.865$ \\
\hline$P W P$ & Danger & .591 & $.212-.780$ & .529 & $.037-.765$ & .560 & $.124-.773$ \\
\hline$A \cup D$ & S.D & .571 & $.304-.713$ & .628 & $.351-.761$ & .599 & $.328-.737$ \\
\hline$P W P$ & S.D & .690 & $.408-.820$ & .657 & $.433-.781$ & .674 & $.421-.801$ \\
\hline$A U D$ & P.S.D & .411 & $-.032-.639$ & .396 & $.013-.616$ & .403 & $-.010-.628$ \\
\hline$P W P$ & P.S.D & .184 & $-.432-.505$ & .461 & $.142-.652$ & .323 & $-.145-.578$ \\
\hline
\end{tabular}

Note. An alpha of .7 to .8 is regarded as acceptable when using a psychometric measure to compare groups of individuals and will be used as the reference value in this study (Bland \& Altman, 1997). Results reported are for the mean bootstrapped alpha and are in the form: $\alpha=X$ [95\% confidence Interval]

\section{Stigma inventory EFA and PCA}

The Principal Component Analysis (PCA) of the Stigma Inventory revealed that for both the PWP and AUD conditions the items loaded maximally in the manner expected, all three dangerousness items loaded onto one factor and the three controllability items loaded onto the second factor, Figure 13 and 14 . The cumulative variance explained by the two-factor solution was $64.189 \%$ for the AUD condition and $63.336 \%$ for the PWP condition, which is generally acceptable ( $>60 \%)$ for measuring this construct, Hair Jnr, Black, Babin \& Anderson, (2014) (Appendix J). 

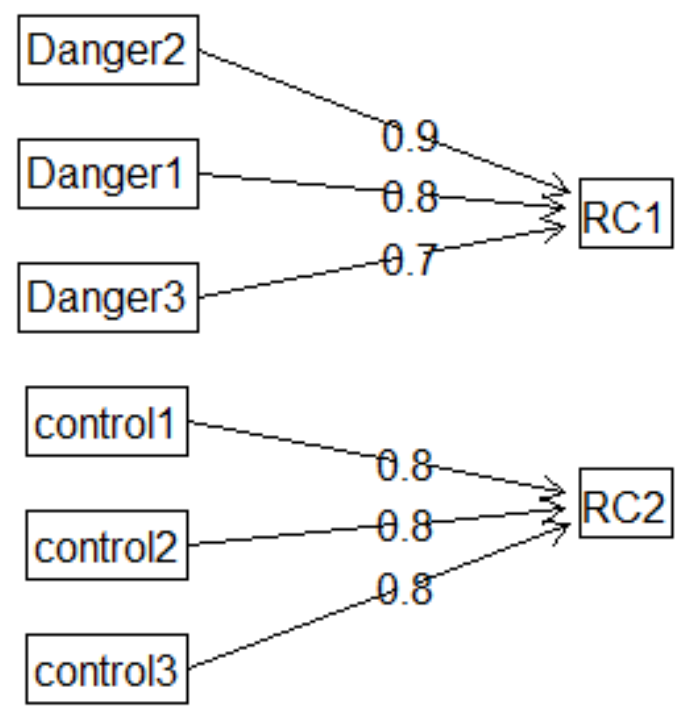

Figure 13

AUD Stigma Inventory PCA Factor Loadings
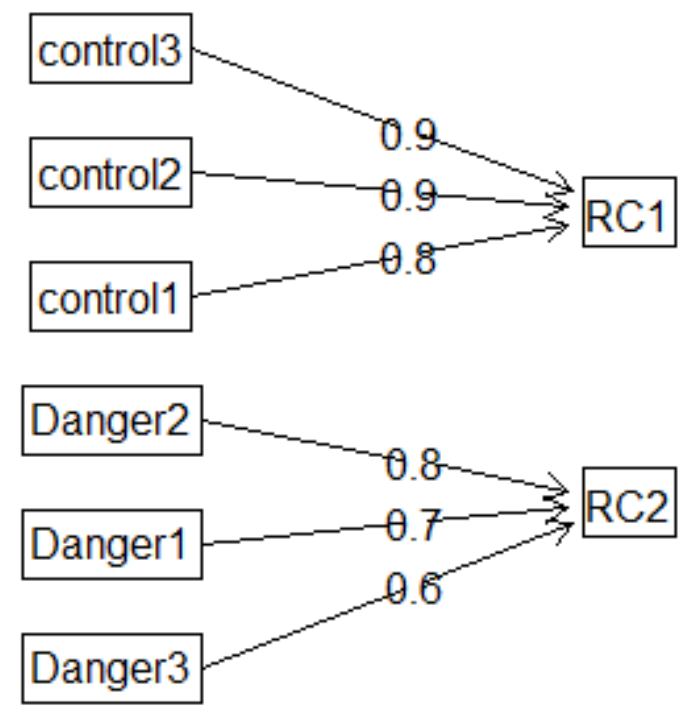

Figure 14

PWP Stigma Inventory PCA Factor Loading 
An Exploratory Factor Analysis (EFA) of the AUD stigma inventory found that the two-factor solution also aligned with the results of the previous researchers, with one factor loading maximally onto each of the dangerousness and controllability categories of questions, Figure 15 . This solution explained $50.418 \%$ of the variance in responses, Appendix J. The EFA of the Stigma Inventory for the PWP condition explained $51.817 \%$ of the variance, Appendix J, in response with a two-factor solution and the factors loaded onto the two categories of questions as expected, Figure 16.

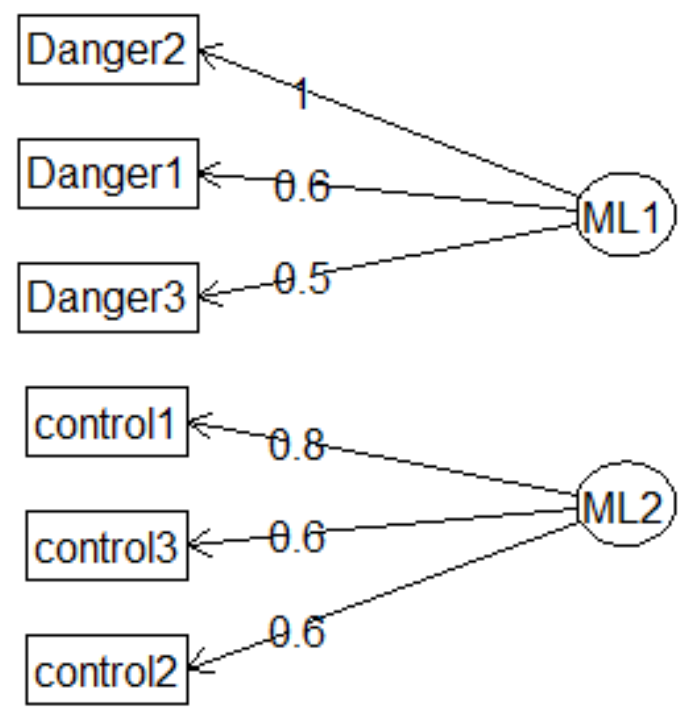

\section{Figure 15}

AUD E.F.A Stigma Inventory Factor Loadings

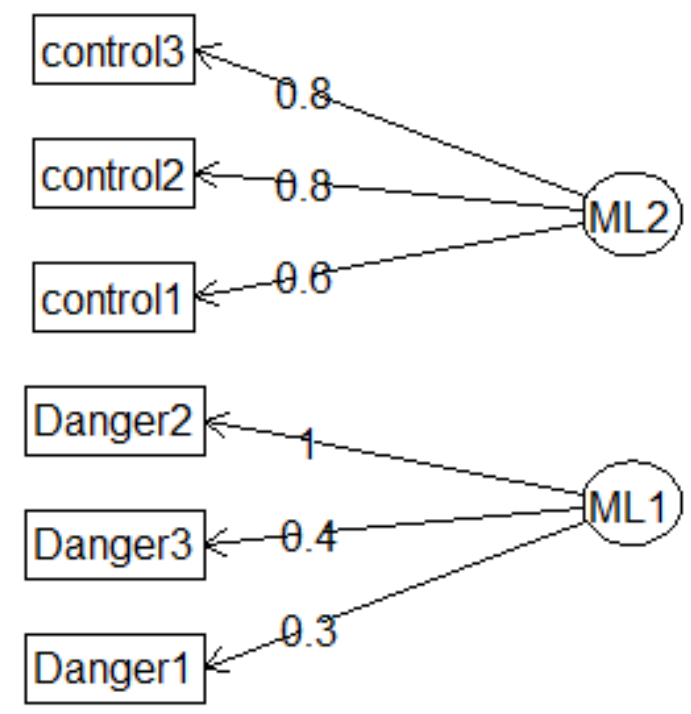

Figure 16

PWP EFA Stigma Inventory Factor Loadings 


\section{Social Distance Scale EFA and PCA}

The Social Distance scale was assessed using a PCA with one factor specified, item 5 did not load onto the factor in either the PWP or AUD condition, Figure 17 and 18, and item 6 did not load onto the factor in the PWP condition, Figure 18. An EFA with a single factor solution did not load as expected for the PWP condition with items 5 and 6 having much lower item loadings than expected $<.3$, Figure 19. The one factor EFA of the Social Distance Scale for the AUD condition did not load in the expected manner at all as item 5 should have a negative loading, Figure 20.

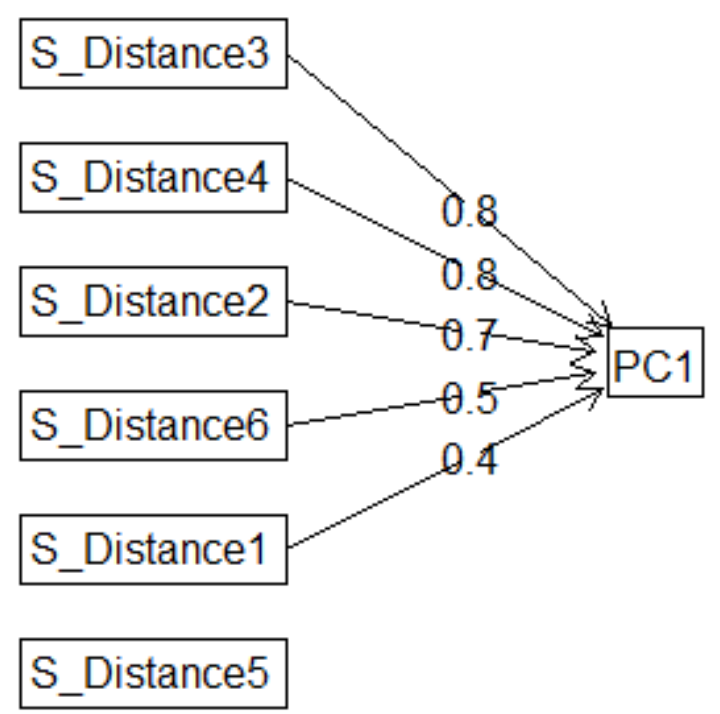

Figure 17

AUD PCA Social Distance Scale Single Factor Item Loadings 


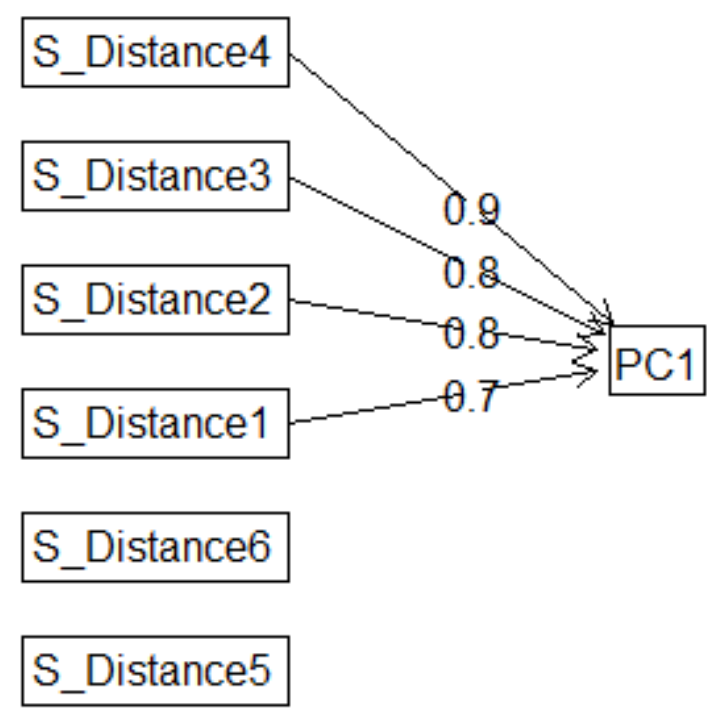

Figure 18

PWP PCA Social Distance Scale Single Factor Item Loadings

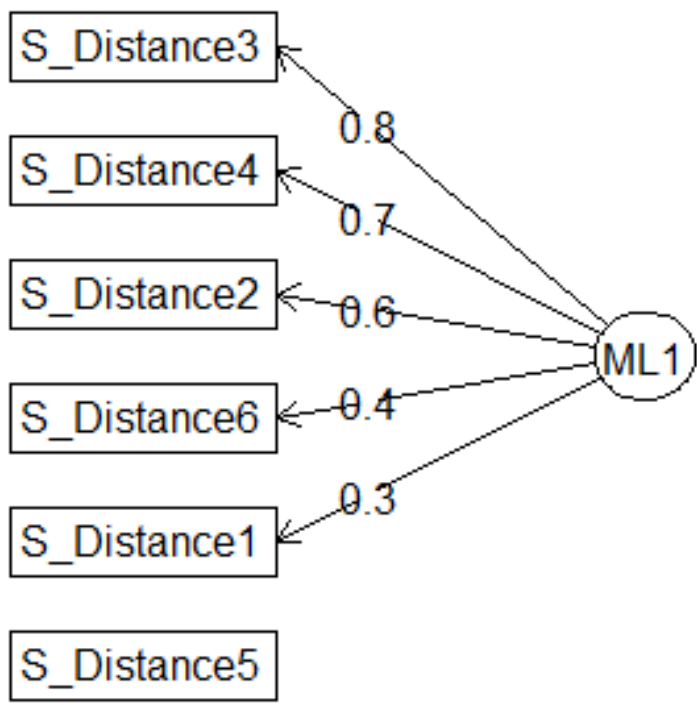

Figure 19

AUD EFA Social Distance Scale Single Factor Loadings 


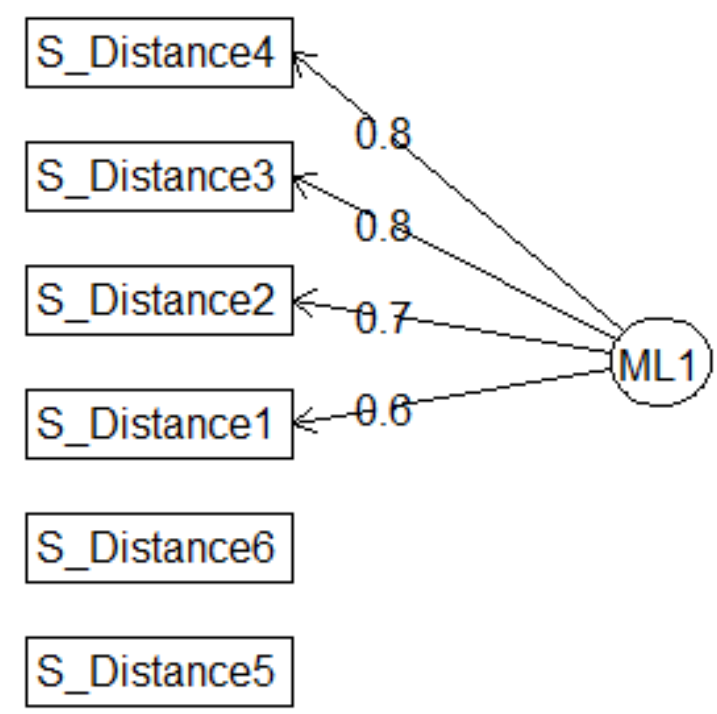

Figure 20

PWP EFA Social Distance Scale Single Factor Item Loadings

Upon further investigation of the Social Distance Scale, a two-factor solution produced much better results for both conditions. The items related to the same factors in both conditions for the PCA, Figure 21 and 22. However, the EFA produced unexpected results for item 6, in the AUD condition, Figure 23. The PWP EFA indicated a consistent structure with the PCA, Figure 24. This two-factor solution also increased the variance in responses by a significant amount for both conditions and analyses, with some being raised over the threshold of $60 \%$ cumulative variance explained, Appendix J. 


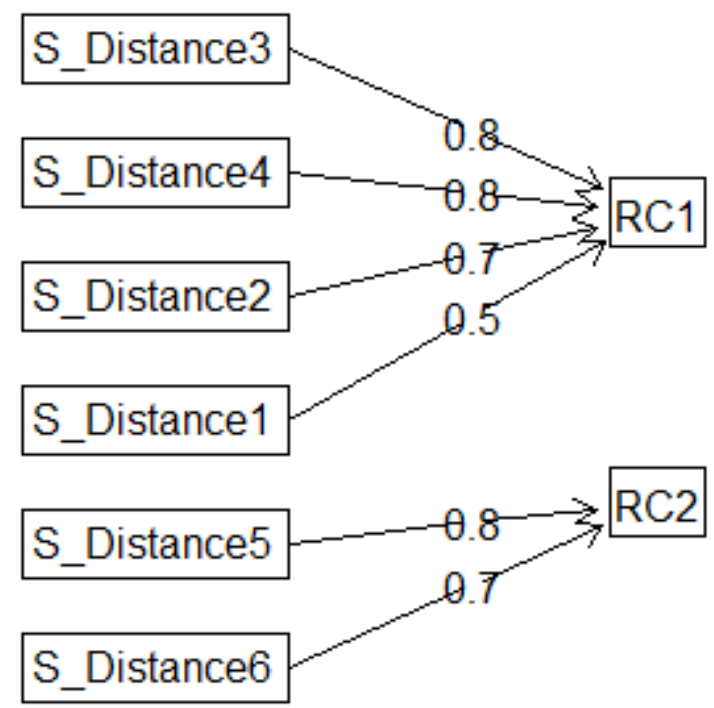

Figure 21

AUD PCA Social Distance Scale Item Loadings with Two-Factors

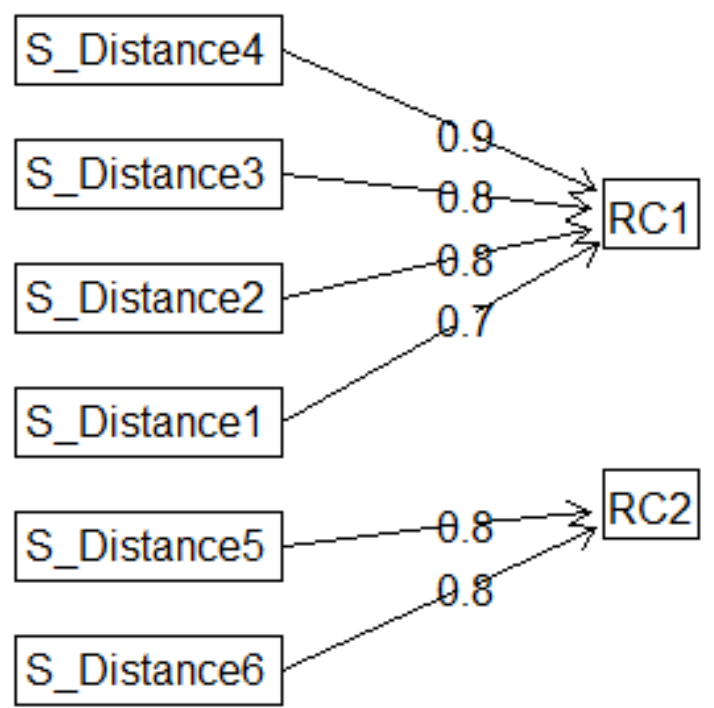

Figure 22

PWP PCA Social Distance Scale Item Loadings with Two-Factors 


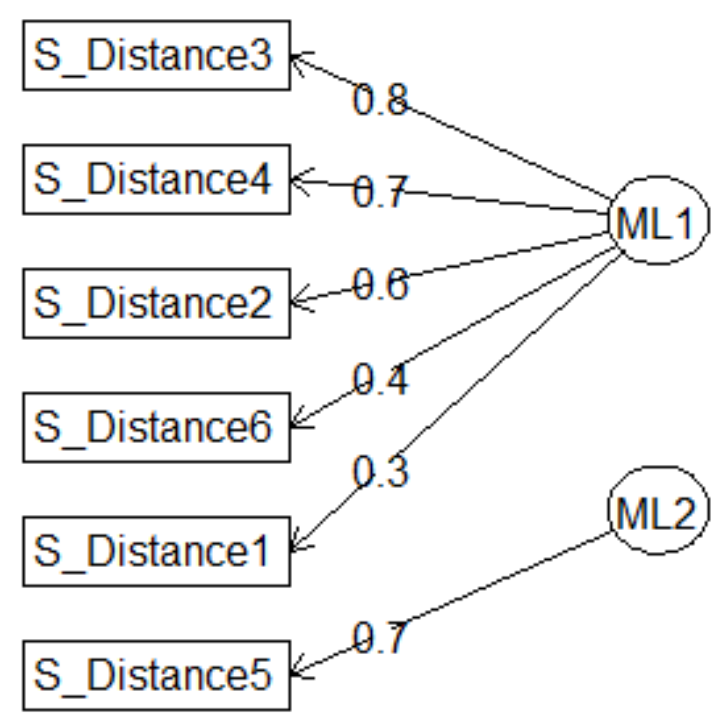

Figure 23

AUD EFA Social Distance Scale Item Loadings with Two-Factors

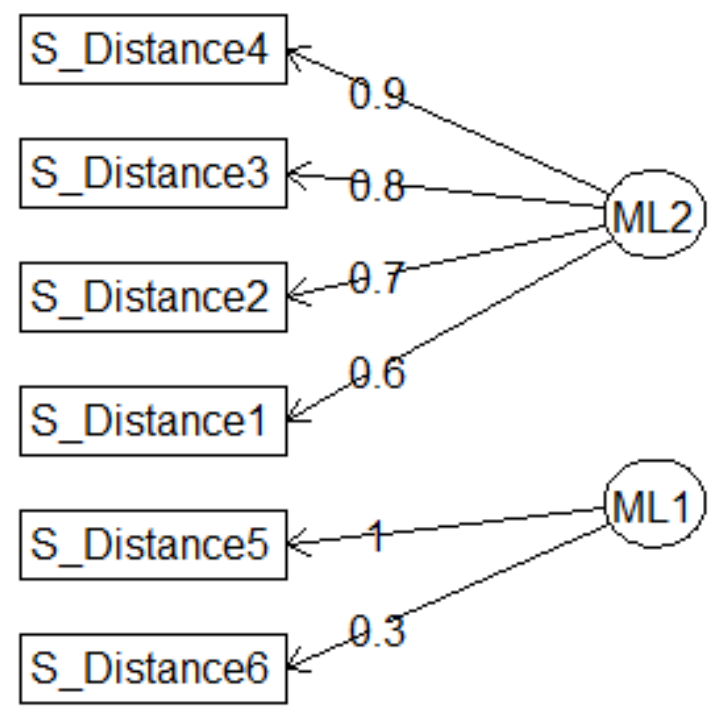

Figure 24

PWP EFA Social Distance Scale Item Loadings with Two-Factors

\section{Perceived Social Distance Scale EFA and PCA}

The single factor PCA for both conditions indicated negative loadings for items 5 and 6 which was expected for these negative valence items, Figure 25 and 26, and the cumulative variance explained 
was $52 \%$ for both, Appendix J. However, the EFA for the AUD condition did not load as expected, Figure 27, although this was not the case with the EFA for the PWP condition, Figure 28. Both one factor EFA solutions explained less variance than the threshold $>60 \%$, Appendix J.

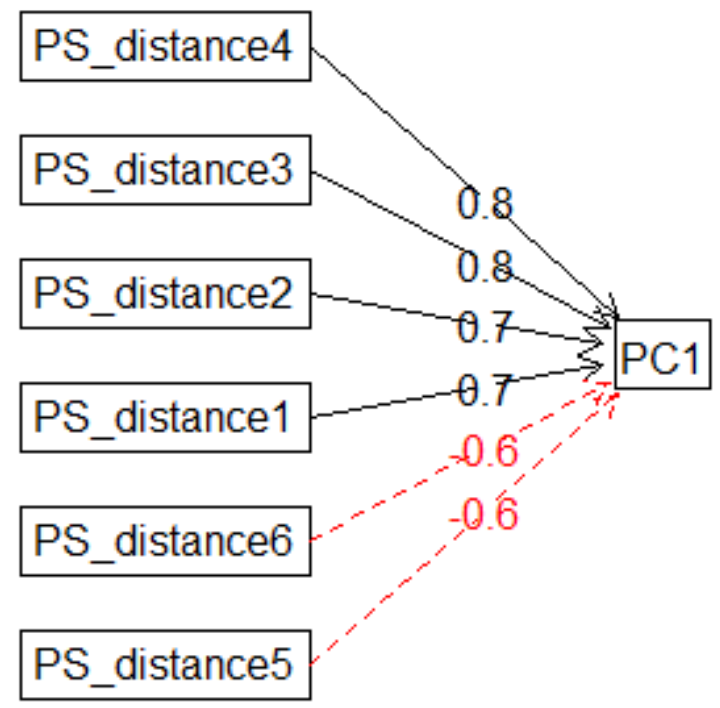

Figure 25

AUD PCA Perceived Social Distance Scale Item Loadings for One Factor

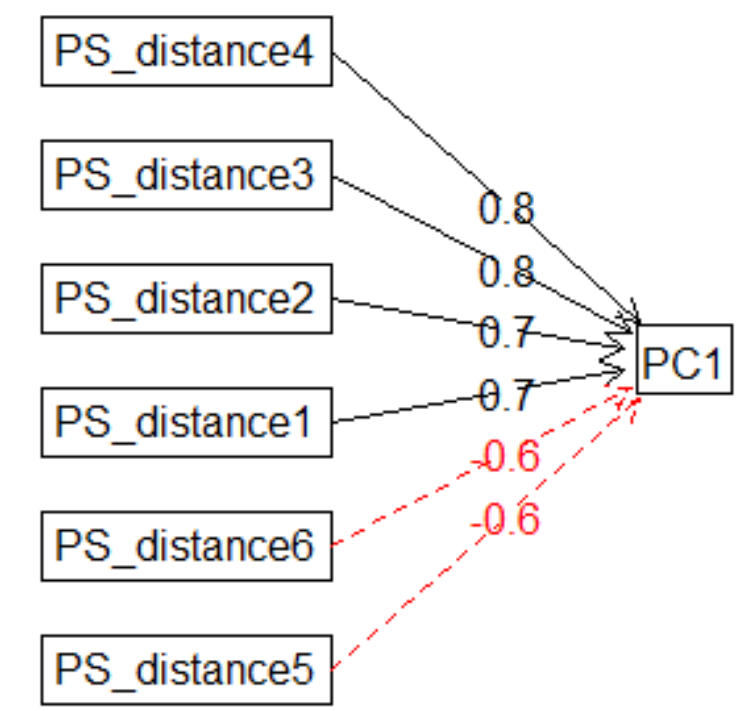

Figure 26

PWP PCA Perceived Social Distance Scale Item Loadings for One Factor 


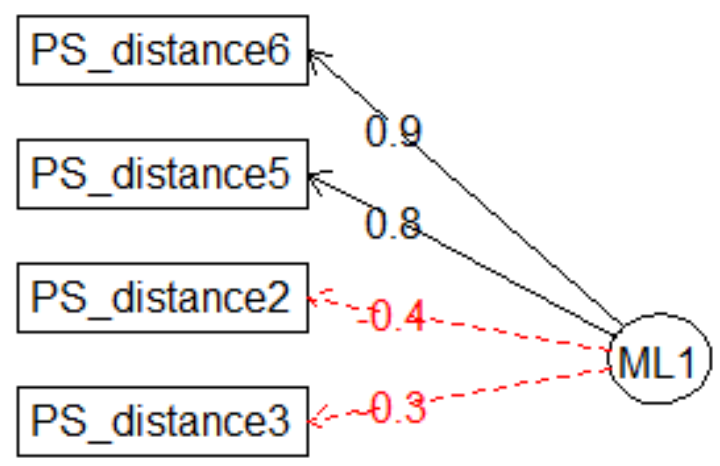

PS_distance4

PS_distance1

Figure 27

AUD EFA Perceived Social Distance Scale Item Loadings for One Factor

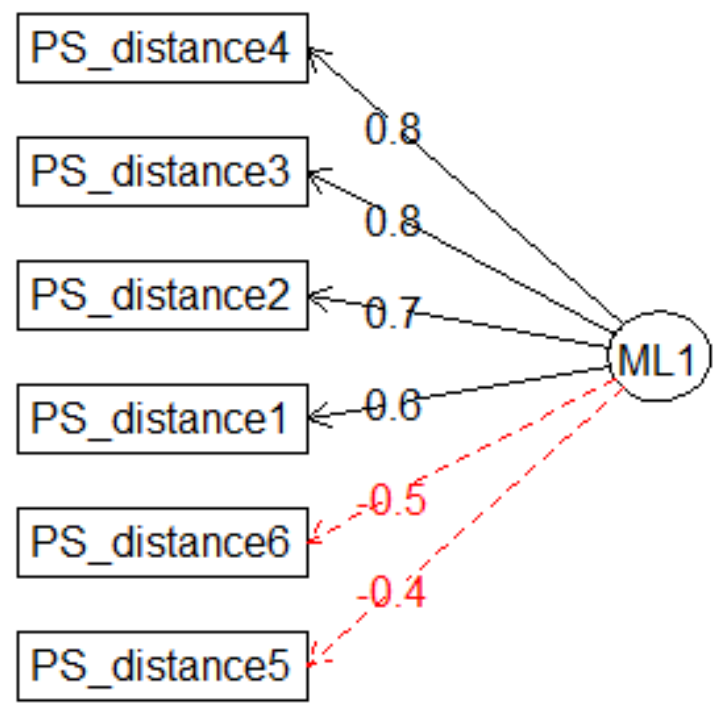

Figure 28

PWP EFA Perceived Social Distance Scale Item Loadings for One Factor

A two-factor solution for the Perceived Social Distance PCA raised the cumulative variance captured to $>60 \%$ for both conditions, Appendix J, and also resulted in the same factor loadings seen in the Social Distance Scale, Figure 29 and 30. The two-factor solution for the EFA for the Perceived Social Distance Scale also results in higher amounts of cumulative variance captured by the model, 
Appendix J, and the factor loadings are much more similar between the conditions, indicating that this is a more suitable solution for comparing scores between these conditions, Figure 31 and 32 .

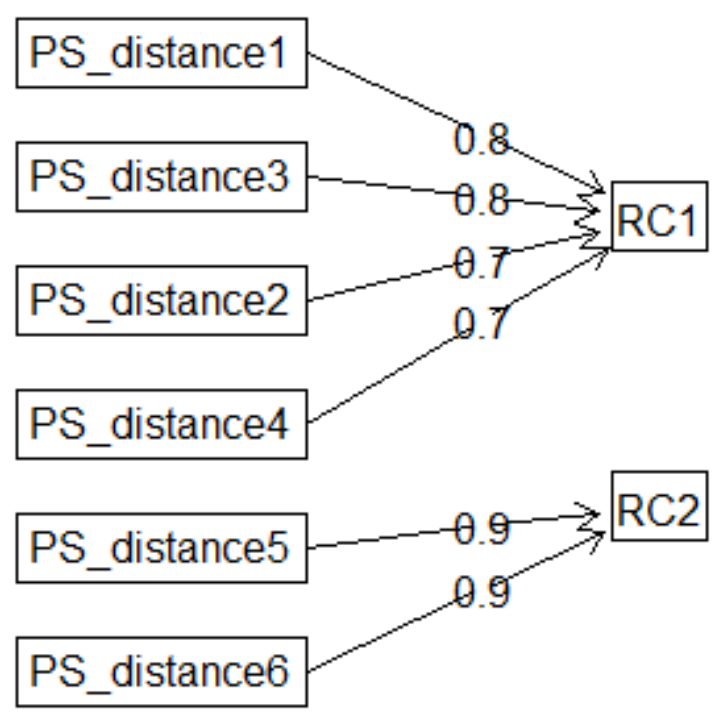

Figure 29

AUD PCA Perceived Social Distance Scale Item Loadings for Two Factors

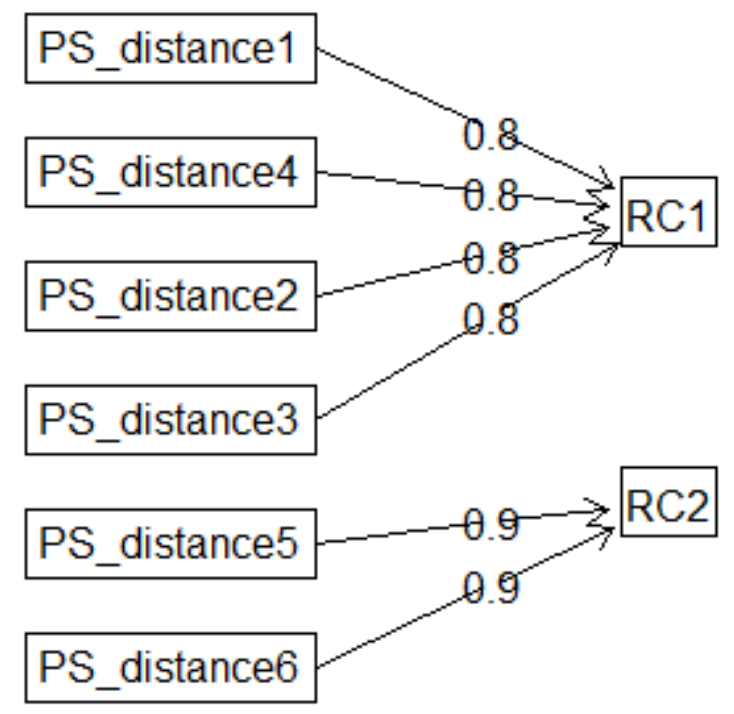

Figure 30

PWP PCA Perceived Social Distance Scale Item Loadings for Two factors 


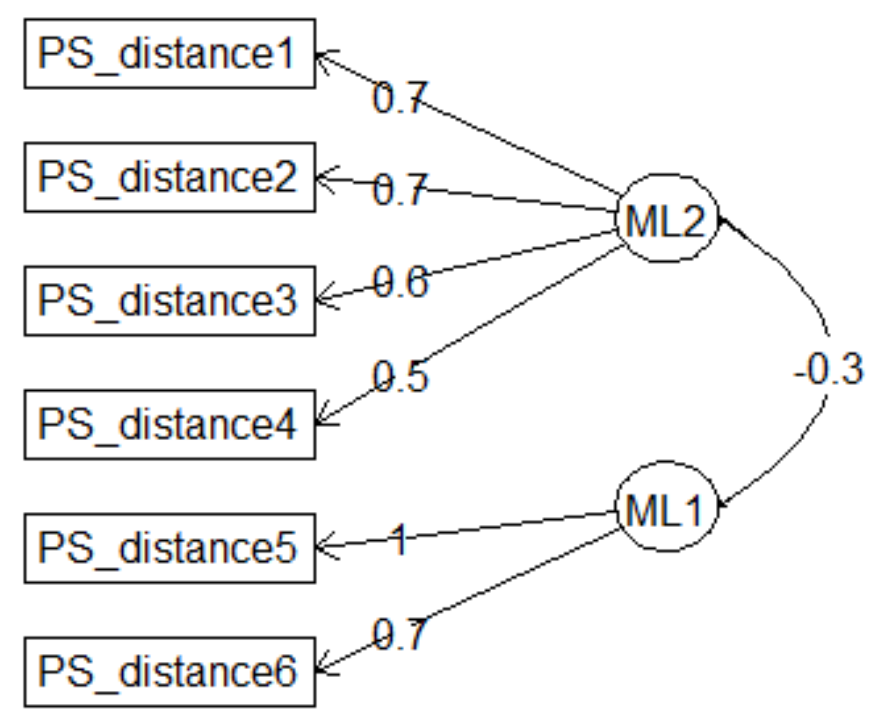

Figure 31

AUD EFA Perceived Social Distance Scale Item Loadings for Two Factors

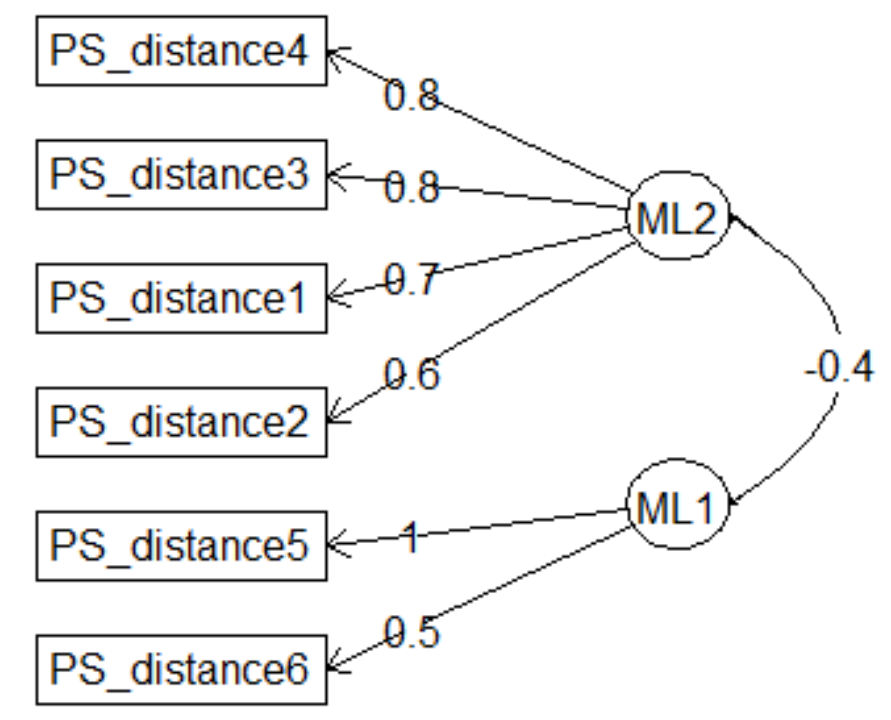

Figure 32

PWP EFA Perceived Social Distance Scale Item Loadings for Two Factors 


\section{Discussion}

\section{Bayesian Ordinal Regression}

\section{Stigma Inventory Results}

This study piloted an intervention towards non-offending paedophiles and the results indicated that there was a decrease in Stigmatism Inventory scores following the intervention. However, this was not a consistent decrease as the credible interval included zero. As such the first hypothesis was not fully supported in this study. However, as a Bayesian analysis was used the distribution of credible estimates could be modelled, Appendix K. From this it can be postulated that there may be a significant effect of this style of intervention, as the estimates peak below zero and the bulk of the estimate distribution is below zero. As such a follow-up study with a larger sample size may have a large enough scope to discern this effect conclusively. Whilst the analysis results were not conclusive, they do support the effects observed in the study by Jahnke, Phillip \& Hoyer (2015), which was the conceptual basis of this research, despite the difference in targeted sample - the original study targeted psychotherapists in training. These results highlight that the reported effectiveness of stigmatism interventions towards non-offending paedophiles could potentially carry over to a public sample.

It was expected that the effect of the intervention would be greater for the PWP condition, Hypothesis 2, and whilst this could not be deduced in this study the posterior probability (.92) was high. In the posterior distribution, Appendix K, it can be observed that the majority of credible estimates fall within the expected range, $<0$, which supports the second hypothesis. Hence, it may be beneficial to replicate this study with a larger sample size as this would decrease the C.I and the current results suggest this would produce a negative C.I estimated difference in intervention effectiveness. This disparate intervention effectiveness was possibly due to the lack of public knowledge about paedophilia, as professional opinion on AUD has greater exposure to the public (McCartan, 2004; McCartan, 2010; Richards, 2017). If professional opinion was better disseminated the interventions should have had a similar degree of influence on the participants.

Previous research indicated that the Stigma Inventory scores in the PWP condition would be higher than the AUD condition (Jahnke, Imhoff \& Hoyer, 2014), which was strongly supported in this study pre-intervention. However, the C.I for the difference in Stigma Inventory scores post intervention included zero. Therefore, post-intervention the estimate for Stigma Inventory scores was not consistently positive despite the stigmatism scores being generally higher for the PWP condition. This may indicate that the stigmatism intervention worked relatively well as it made differentiating 
between the two stigmatised groups less conclusive. Alternatively, it may be an indication that the study required more participants, as the majority of estimates for the differences in stigmatism scores post intervention were positive, and only $10 \%$ of the distribution of hypothesis 4 estimates were equal to or below zero. Therefore, with a larger sample size the C.I could be reduced and allow for more conclusive results regarding hypothesis four. The evidence gathered in this study suggests that with a larger sample size the estimate would be clearly positive, supporting the fourth hypothesis. These results support the views of the literature around the comparative stigmatization of non-offending paedophilia with other highly stigmatized groups (Imhoff \& Jahnke, 2017; Jahnke, Imhoff \& Hoyer, 2014; Levenson \& Grady, 2019).

The participant group-level estimates indicated that the intervention effect was dependent on the participant. This may be an overestimate due to the relatively small sample size or it could be an indication that the effectiveness of the intervention is dependent on an unmeasured demographic variable. For instance, an exploratory study by Jahnke, Imhoff and Hoyer (2014) found that political beliefs and education level attainment significantly correlated with controllability and dangerousness responses. This may account for some of the variation in intervention effectiveness. The item group-level estimates indicated that there was a differing pattern of responses dependent on the experimental condition. This was expected as previous research indicated that there are item level differences in responses when measuring stigmatism towards AUD and PWP groups (Jahnke, Imhoff \& Hoyer, 2014)

Overall the Stigmatism Inventory scores in this study were significantly higher pre-intervention, and whilst most estimates indicated this was also the case post-intervention more data would be required to confirm this. The Intervention also reduced stigmatism scores towards PWP in most instances and this effect was greater for the PWP condition. However, a larger survey would be required to confirm if either of these intervention effects were consistent.

\section{Social Distance Scale and Perceived Social Distance Scale Results}

The Social Distance Scale and Perceived Social Distance Scales did not exhibit a significant difference in scores between the AUD and PWP conditions pre-intervention as expected. In Hypothesis 3 it was expected that there would be higher Social Distance scores for the PWP condition pre-intervention, however, this was true for only $13 \%$ of the credible estimates. This indicated that either: there was no difference in Social Distance scores; or that the PWP scores were consistently lower than those for the AUD condition. The distribution of estimates for hypothesis 3 indicated that the latter would be more likely with a larger sample, Appendix $\mathrm{K}$. This is at odds with the current research that purports the heightened social distance scores towards non-offending 
paedophiles within the public and professional communities (Jahnke, Imhoff, \& Hoyer,2014; Jahnke, Philipp, \& Hoyer, 2015; Klapilová, et al., 2019; Levenson \& Grady, 2019 McCarton, 2010).

Perceived Social Distance scores were found to be higher for the PWP condition pre-intervention indicated by the $61 \%$ of the hypothesis three' estimate distribution that was positive, Appendix K, however as the C.I included zero and as $39 \%$ of the estimates were negative these results are inconclusive (Jahnke, Imhoff, \& Hoyer,2014; Jahnke, Philipp, \& Hoyer, 2015; Klapilová, et al., 2019; Levenson \& Grady, 2019; McCarton, 2010).

The intervention had no conclusive effect for the PWP Social Distance Scores, $85 \%$ of the estimate distribution was positive and the C.I included zero. As such, it is likely that the intervention increased scores rather than decreasing them as predicted by Hypothesis 1, as indicated by the hypothesis estimate distribution, Appendix K. This would have to be investigated with a larger sample to be confirmed and these results are not supported by the literature (Jahnke, Phillip \& Hoyer, 2015; Levenson \& Grady, 2019).

This may be due to the video material involved in the PWP intervention as it may have resulted in a framing-effect that led to the participants considering social distance in an unexpected manner. The goal of the intervention video was to create a narrative about the subject using a counterstereotypical exemplar which has been shown to be effective in challenging stigmatism (Brown, 2019; Janoušková et al., 2017). However, as the individual in the video is a middle-aged white male with thinning unkempt hair the participants may have resorted to a stereotyping-frame as the narrator typified their stereotypical view of what a paedophile or sex-offender would look like (Bowman, 2018; Harper \& Bartels, 2016). By failing to come across as a counter-stereotypical example of the group participants may be inclined to apply a stereotyping frame such as social categorization or legitimate victimization which would in turn maintain and reinforce stigmatizing beliefs (Yang, 2015). This speculation is validated by the differing results achieved by Jahnke, Imhoff \& Hoyer (2015) as the individual in their video material has been anonymised and defeatured which prevented the participants from applying a stereotyped frame based on the appearance of the subject.

75\% of the Perceived Social Distance hypothesis 1 distribution estimates were negative indicating that the intervention decreased scores in most cases, Appendix K. However, as the C.I includes zero the null hypothesis that the intervention had no effect cannot be rejected and so Hypothesis 1 cannot be resolved without further study. However, the results support the effects observed in intervention literature surrounding non-offending paedophilia, as they suggest that with a larger sample size the true effect of the intervention would be negative (Jahnke, Imhoff \& Hoyer, 2015). 
The comparative intervention effectiveness results indicate that there is no discernible difference in intervention effect between conditions for the Social Distance scale. As such, it is plausible that the intervention may have been more effective for the AUD group. However, the credible interval includes zero, therefore, the null hypothesis that the intervention had an equally sized effect across conditions, cannot be discredited. Combined with the fact that the intervention 1 estimate C.I highlights that the intervention did not consistently reduce social distance scores, there is reason to believe that the intervention was ineffective for both conditions.

Participant group-level effects for social distance indicated that the effect of the intervention varied dependent on the participant. However, as there was a possible issue in establishing a counterstereotypical exemplar in the PWP intervention video this variation cannot be attributed solely to participant level variations such as education or political ideology (Jahnke, Imhoff \& Hoyer, 2014). Similarly, the group-level effects for items indicated that there was a variation in item response patterns between intervention times and between intervention times within the PWP condition. However, these estimates of variability suffer from the same methodological issue as the participant group-level effects. As such, the group-level effects for items may reflect changes due to a stereotype frame-effect rather than the intended intervention effect (Yang, 2015).

The intervention had a greater effect on the PWP Perceived Social Distance scores, when compared to the AUD condition. This was indicated by the high proportion, $85 \%$, of the hypothesis 2 estimates that were $<0$, Appendix K. As such, it is likely the intervention had a unique effect dependent on condition. However, as the C.I includes zero the null hypothesis, that the intervention was equally effective across conditions, cannot be rejected. Extending this study and gathering a larger sample, greater acuity in the C.I could be achieved and that would allow a more decisive conclusion to be drawn, which the current results suggest would favour the hypothesised negative effect. This differing effectiveness could be attributed to the lack of public knowledge on the topic of paedophilia as without sufficient information an inaccurate opinion will be drawn on the perception of peers (McCartan, 2004; McCartan, 2010; Richards, 2017).

The participant group-level effects of the intervention for the perceived social distance scale had a C.I that included zero and so the degree of variance attributable to participant level effects for the intervention effect were inconclusive. The item group-level effects indicated that there was a small degree of item variance attributable to the condition, however, the C.I for item response variance across interventions and for the interaction between condition and intervention approached zero. As such the degree of item level variance attributable to the intervention cannot be discerned in this study. 
Post intervention PWP Social Distance score estimates were positive in $30 \%$ of cases (Appendix K). As, the C.I includes zero, these results indicate that either: the PWP scores were lower than the AUD scores post intervention; or that there was no difference in scores between the conditions postintervention. Post-intervention PWP Perceived Social Distance score estimates were positive in 39\% of cases, Appendix K, the C.I in this case also includes zero. As such there is no conclusive evidence regarding Hypothesis 4, that PWP scores would be higher post-intervention. For both the Social Distance and Perceived Social Distance scales a larger sample size is required to draw more decisive conclusions about the difference in scores between the PWP and AUD condition.

\section{Questionnaire Analyses}

\section{Stigma Inventory PCA, EFA and Alpha Results}

The Stigma Inventory Scale bootstrapped alpha results were adequate in respect of the recommended $\alpha>.7$ threshold, Bland \& Altman (1997), except for the dangerousness factor in the PWP condition. This may be due to the age-related aspect of the dangerousness items as previous studies indicate that there is a persistently higher rating for the danger towards children item (Appendix A, item 4) for the PWP condition, (Jahnke, Imhoff, \& Hoyer, 2014). In comparison the AUD dangerousness factor item responses differ marginally across the range of ages: child, adolescent and adult.

The component structure of the Stigma Inventory produced the expected results as a twocomponent solution resulted in one component for controllability items and one for dangerousness items. The EFA produced a similar structure of factor loadings as expected. The cumulative variance accounted for by the PCA was $>60 \%$ for both conditions which is the generally accepted threshold for measuring a construct for research purposes, Babin \& Anderson (2014). This re-validation of the English translation of the Stigma Inventory validates the results of the American stigmatism survey conducted by Jahnke, Imhoff \& Hoyer (2014), as they did not re-assess the translated scale. This confirmation of the scale structure will also support its use in future studies around the topic of nonoffending paedophilia.

\section{Social Distance and Perceived Social Distance EFA, PCA and Alpha Results}

The bootstrapped alpha results for the Social Distance scale and Perceived Social Distance scale were all below the threshold decided upon for this research, $\alpha>$.7. While Social Distance scale results were close to the desired threshold, AUD $\alpha=.599$, PWP $\alpha=.674$, the results for the Perceived Social Distance scales were inadequate, Table 11. As such the Perceived Social Distance scale likely requires re-structuring to improve its reliability before use in future studies. This finding 
may be problematic for the research by Jahnke, Imhoff \& Hoyer (2014) as there was no reliability analysis undertaken for the Perceived Social Distance scale before its use in the study.

The single component PCA of the Social Distance scale did not result in the item loadings that were expected. The problems seemed to be due to Items 5 and 6 , Appendix B, as they tended to have component loadings $<.3$. Due to this issue, the cumulative variance captured by the one factor models was, for both conditions, unacceptably low. Further analysis with a two-component solution produced a stable structure of item loadings for the PCA for both conditions and led to a marked improvement in the cumulative variance accounted for by the models. In both conditions items 5 and 6 maximally loaded onto a single component. The two-factor EFA also resulted in a greater cumulative variance accounted for. However, the two-factor AUD EFA did not produce a similar structure to the two-component PCAs or the two-factor PWP EFA, which is anomalous.

As the Perceived Social Distance scale contains items with the same wording as the Social Distance scale it is beneficial to consider the results of both scales EFA and PCA analyses before investigating why there are anomalous item and factor loadings.

The single component Perceived Social Distance PCA resulted in items 5 and 6 producing negative component loadings, as expected with negative valence items, for both conditions. The single factor EFA produced the same structure of results with negative item loadings for item 5 and 6 for the PWP condition. However, the single factor EFA for the AUD condition did not produce the expected item loadings at all.

Further analysis with a two-component PCA for the Perceived Social Distance scale resulted in higher cumulative variance being captured by the model and both conditions exhibited the same pattern of loadings. Notably, this was also the case for the two-factor EFA. In both the EFA and PCA a single factor/component is maximally associated with items 5 and 6.

Thus, further analysis of the Social Distance and Perceived Social Distance scales indicates that items 5 and 6 are weakly related to the latent underlying construct being measured, which is assumed to be social distance by the creators of the scale (Jahnke, Imhoff \& Hoyer, 2014). This is evidenced by their underwhelming relationship to the single component/factor solutions. It should be noted that items 5 and 6 are novel items added to the scale originally developed by Bogardus (1933) by Jahnke, Imhoff \& Hoyer (2014) to make the scale suitable for the topic of non-offending paedophiles. As such, they have a disparate evidence base for their relationship to social distance, whereas items 1-4 have been re-validated in numerous studies (Wark \& Galliher, 2007). Therefore, items 5 and 6 may be measuring another latent construct. As these items ask the participant if they 
believe members of the group in question should be in jail and if they should be killed, they may be measuring punitive attitudes instead of social distance. Therefore, removing items 5 and 6 would make both scales much more reliable and valid measures of social distance and perceived social distance. A possible substitution for items 5 and 6 may ask; "I would rather these people did not live in my country" or "these people should not be allowed in this country", both of which emulate the exclusion style of question featured at the end of the scale Jahnke, Imhoff \& Hoyer (2014) used as the basis for their Social Distance scale (Geisinger, 2010).

\section{Limitations}

This study was limited by the design of the Social Distance and Perceived Social Distance scales. This created issues with the Bayesian Ordinal Regression models fitted as the cumulative family specified within the model assumes that there is only one latent construct being measured. The PCAs and EFAs suggested that the latent construct was not limited to social distance in the scales but extended to punitive attitudes, which has led to a construal of the results. Another issue was that the video material was not anonymised and as such the social distance measures may have suffered as a result due to a stereotype framing-effect. The last, and most apparent, limitation was a lack of participants. It was difficult to convince individuals to provide their opinion on the subject of paedophilia as the subject is particularly taboo and some individuals were concerned about being seen as sympathetic irrespective of the anonymity provided by the study design.

Future research could improve on this study by anonymising the exemplar in the intervention material and gain a more accurate measure of social distance and perceived social distance by excluding the $5^{\text {th }}$ and $6^{\text {th }}$ items from the scales used in this study. A hypothetical question about willingness to seek therapy if they suffered from the disorder may be beneficial, as this could provide an indication of intervention effectiveness with respect to perceived formal help-seeking acceptability. Furthermore, a larger sample size would be recommended to reduce the C.I. This may be achieved by recruiting individuals in public, through collaboration with the vir-ped online organisation or the NSPCC charity research team.

\section{Conclusions}

This study has piloted the first U.K public stigmatism intervention for paedophilia. The Social Distance and Perceived Social Distance scales were found to have an inadequate factor structure and reliability. As such, the lack of support they provided for the hypotheses should be interpreted tentatively. The Stigma Inventory scale provided reliable measurements of the stigmatism towards individuals with AUD and PWP. The Bayesian Ordinal Regression results indicated that there was a 
definite higher level of stigmatism towards PWP pre-intervention supporting the third hypothesis. While the other hypotheses of this research were not as well supported by the Stigma Inventory results the Bayesian methodology allowed for a much more robust estimation of whether the hypotheses were falsifiable. The estimate distributions for Hypotheses 1, 2 and 4 all tended towards the expected outcomes for the Stigma Inventory. This emphasises the need for further study within this area as our analysis, whilst not conclusive, strongly suggests that if the study was replicated with a larger sample size each of the hypothesised results would be well supported.

The evidence drawn from the Social Distance scale revealed that the data tended away from the results of other researchers who have performed similar research in other countries. As such, further investigation into this would be required to gain a better understanding of public opinion in the U.K before a stigmatism intervention was used on the wider public. This was also the case for the Perceived Social Distance scale.

Overall, this study has shown the potential effectiveness of a public stigmatism intervention in the U.K. Whilst a solid confirmation cannot be drawn from these results the data gathered does not indicate that the development of such an intervention is unfeasible. This pilot provides the necessary first step into developing an intervention that could be applied to the whole of the U.K, with minimal cost, to resolve the severe stigmatism that has been created by the media. This may provide a developmental pathway which could decrease the number of CSA offences, and its financial burden, through the legitimisation of paedophilia as a disorder, allowing the full potential of secondary prevention to be realised. 


\section{References}

Beier, K., Grundmann, D., Kuhle, L., Scherner, G., Konrad, A., \& Amelung, T. (2015). The German Dunkelfeld Project: A Pilot Study to Prevent Child Sexual Abuse and the Use of Child Abusive Images. The Journal Of Sexual Medicine, 12(2), 529-542. doi: 10.1111/jsm.12785

Bland, J., \& Altman, D. (1997). Statistics notes: Cronbach's alpha. BMJ, 314(7080), 572-572. doi: 10.1136/bmj.314.7080.572

Bogardus, E. S. (1933). A social distance scale. Sociology \& Social Research, 17, 265-271.

Bowman, K. N. (2018). University Students' Perceptions of Child Sex Offenders (Doctoral dissertation, University of Colorado Colorado Springs).

Brown, J., \& Saied-Tessier, A. (2015). Preventing Child Sexual Abuse. London:NSPCC.

Brown, S. (2019). The Effectiveness of Two Potential Mass Media Interventions on Stigma: VideoRecorded Social Contact and Audio/Visual Simulations. Community Mental Health Journal, 56(3), 471-477. doi: 10.1007/s10597-019-00503-8

Bürkner, P., \& Vuorre, M. (2019). Ordinal Regression Models in Psychology: A Tutorial. Advances In Methods And Practices In Psychological Science, 2(1), 77-101. doi: 10.1177/2515245918823199

Cantor, J., \& McPhail, I. (2016). Non-offending Pedophiles. Current Sexual Health Reports, 8(3), 121128. doi: 10.1007/s11930-016-0076-z

Conti, G., Morris, S., Melnychuk, M., \& Pizzo, E. (2017) The economic cost of child maltreatment in the UK: a preliminary study. London: NSPCC

Currie, J., \& Tekin, E. (2012). Understanding the Cycle: Childhood Maltreatment and Future Crime. Journal Of Human Resources, 47(2), 509-549. doi: 10.1353/jhr.2012.0017

Daykin, A., \& Moffatt, P. (2002). Analyzing Ordered Responses: A Review of the Ordered Probit Model. Understanding Statistics, 1(3), 157-166. doi: 10.1207/s15328031us0103_02

Depaoli, S., \& van de Schoot, R. (2017). Improving transparency and replication in Bayesian statistics: The WAMBS-Checklist. Psychological Methods, 22(2), 240-261. doi: 10.1037/met0000065

Doidge, J., Higgins, D., Delfabbro, P., \& Segal, L. (2017). Risk factors for child maltreatment in an Australian population-based birth cohort. Child Abuse \& Neglect, 64, 47-60. doi: 10.1016/j.chiabu.2016.12.002 
Dymond, H., \& Duff, S. (2020). Understanding the lived experience of British non-offending paedophiles. The Journal Of Forensic Practice, ahead-of-print(ahead-of-print). https://doi.org/10.1108/jfp-10-2019-0046

Feldman, D., \& Crandall, C. (2007). Dimensions of Mental Illness Stigma: What About Mental Illness Causes Social Rejection?. Journal Of Social And Clinical Psychology, 26(2), 137-154. doi: 10.1521/jscp.2007.26.2.137

Fergusson, D., Swain-Campbell, N., \& Horwood, J. (2004). How does childhood economic disadvantage lead to crime?. Journal Of Child Psychology And Psychiatry, 45(5), 956-966. doi: 10.1111/j.1469-7610.2004.t01-1-00288.x

Fryda, C., \& Hulme, P. (2014). School-Based Childhood Sexual Abuse Prevention Programs. The Journal Of School Nursing, 31(3), 167-182. doi: 10.1177/1059840514544125

Geisinger, K. (2010). Bogardus Social Distance Scale. The Corsini Encyclopedia Of Psychology. doi: 10.1002/9780470479216.corpsy0135

Grady, M., Levenson, J., Mesias, G., Kavanagh, S., \& Charles, J. (2019). "I can't talk about that": Stigma and fear as barriers to preventive services for minor-attracted persons. Stigma And Health, 4(4), 400-410. doi: 10.1037/sah0000154

Grasso, D., Saunders, B., Williams, L., Hanson, R., Smith, D., \& Fitzgerald, M. (2013). Patterns of Multiple Victimization Among Maltreated Children in Navy Families. Journal Of Traumatic Stress, 26(5), 597-604. doi: 10.1002/jts.21853

Hair Jnr, J., Black, W., Babin, B., \& Anderson, R. (2014). Multivariate data analysis. Essex: Pearson Education.

Harper, C., \& Bartels, R. (2016). The influence of implicit theories and offender characteristics on judgements of sexual offenders: a moderated mediation analysis. Journal Of Sexual Aggression, 23(2), 139-150. https://doi.org/10.1080/13552600.2016.1250963

Harper, C., Bartels, R., \& Hogue, T. (2016). Reducing Stigma and Punitive Attitudes Toward Pedophiles Through Narrative Humanization. Sexual Abuse: A Journal Of Research And Treatment, 107906321668156. doi: 10.1177/1079063216681561

Hébert, M., Langevin, R., \& Oussaïd, E. (2018). Cumulative childhood trauma, emotion regulation, dissociation, and behavior problems in school-aged sexual abuse victims. Journal Of Affective Disorders, 225, 306-312. doi: 10.1016/j.jad.2017.08.044 
Home Office (2019) Police recorded crime and outcomes open data tables. London: Home Office. https://www.gov.uk/government/statistics/police-recorded-crime-open-data-tables

Horn, J., Eisenberg, M., Nicholls, C., Mulder, J., Webster, S., \& Paskell, C. et al. (2015). Stop It Now! A Pilot Study Into the Limits and Benefits of a Free Helpline Preventing Child Sexual Abuse. Journal Of Child Sexual Abuse, 24(8), 853-872. doi: 10.1080/10538712.2015.1088914

Imhoff, R., \& Jahnke, S. (2017). Determinants of Punitive Attitudes Toward People with Pedophilia: Dissecting Effects of the Label and Intentionality Ascriptions. Archives Of Sexual Behavior, 47(2), 353-361. doi: 10.1007/s10508-017-1048-8

Janoušková, M., Tušková, E., Weissová, A., Trančík, P., Pasz, J., Evans-Lacko, S., \& Winkler, P. (2017). Can video interventions be used to effectively destigmatize mental illness among young people? A systematic review. European Psychiatry, 41, 1-9. doi: 10.1016/j.eurpsy.2016.09.008

Jahnke, S., Schmidt, A., Geradt, M., \& Hoyer, J. (2015). Stigma-related stress and its correlates among men with pedophilic sexual interests

Jahnke, S., \& Hoyer, J. (2013). Stigmatization of People With Pedophilia: A Blind Spot in Stigma Research. International Journal Of Sexual Health, 25(3), 169-184. doi: $10.1080 / 19317611.2013 .795921$

Jahnke, S., Imhoff, R., \& Hoyer, J. (2014). Stigmatization of People with Pedophilia: Two Comparative Surveys. Archives Of Sexual Behavior, 44(1), 21-34. doi: 10.1007/s10508-014-0312-4

Jahnke, S., Philipp, K., \& Hoyer, J. (2015). Stigmatizing attitudes towards people with pedophilia and their malleability among psychotherapists in training. Child Abuse \& Neglect, 40, 93-102. doi: 10.1016/j.chiabu.2014.07.008

Klapilová, K., Demidova, L., Elliott, H., Flinton, C., Weiss, P., \& Fedoroff, J. (2019). Psychological treatment of problematic sexual interests: cross-country comparison. International Review of Psychiatry, 31(2), 169-180. doi: 10.1080/09540261.2019.1591353

Lai, C., Marini, M., Lehr, S., Cerruti, C., Shin, J., \& Joy-Gaba, J. et al. (2014). Reducing implicit racial preferences: I. A comparative investigation of 17 interventions. Journal Of Experimental Psychology: General, 143(4), 1765-1785. doi: 10.1037/a0036260

Lewis, T., McElroy, E., Harlaar, N., \& Runyan, D. (2016). Does the impact of child sexual abuse differ from maltreated but non-sexually abused children? A prospective examination of the impact of 
child sexual abuse on internalizing and externalizing behavior problems. Child Abuse \& Neglect, 51, 31-40. doi: 10.1016/j.chiabu.2015.11.016

Levenson, J., \& Grady, M. (2018). Preventing Sexual Abuse: Perspectives of Minor-Attracted Persons About Seeking Help. Sexual Abuse, 31(8), 991-1013. doi: 10.1177/1079063218797713

Levenson, J., \& Grady, M. (2019). "I Could Never Work With Those People ... ": Secondary Prevention of Child Sexual Abuse Via a Brief Training for Therapists About Pedophilia. Journal Of Interpersonal Violence, 34(20), 4281-4302. doi: 10.1177/0886260519869238

Levenson, J., Willis, G., \& Vicencio, C. (2017). Obstacles to Help-Seeking for Sexual Offenders: Implications for Prevention of Sexual Abuse. Journal Of Child Sexual Abuse, 26(2), 99-120. https://doi.org/10.1080/10538712.2016.1276116

Liddell, T., \& Kruschke, J. (2018). Analyzing ordinal data with metric models: What could possibly go wrong?. Journal Of Experimental Social Psychology, 79, 328-348. doi: 10.1016/j.jesp.2018.08.009

Liu, I., \& Agresti, A. (2005). The analysis of ordered categorical data: An overview and a survey of recent developments. Test, 14(1), 1-73. doi: 10.1007/bf02595397

Malvaso, C., Delfabbro, P., \& Day, A. (2017). The child protection and juvenile justice nexus in Australia: A longitudinal examination of the relationship between maltreatment and offending. Child Abuse \& Neglect, 64, 32-46. doi: 10.1016/j.chiabu.2016.11.028

McCartan, K. (2004). 'Here There Be Monsters':The public's perception of paedophiles with particular reference to Belfast and Leicester. Medicine, Science And The Law, 44(4), 327-342. doi: $10.1258 /$ rsmmsl.44.4.327

McCartan, K. (2010). Student/trainee-professional implicit theories of paedophilia. Psychology, Crime \& Law, 16(4), 265-288. doi: 10.1080/10683160802614144

McElreath, R. (2016). Statistical rethinking. Boca Raton: CRC Press, Taylor \& Francis Group.

McKinley, T., Morters, M., \& Wood, J. (2015). Bayesian Model Choice in Cumulative Link Ordinal Regression Models. Bayesian Analysis, 10(1), 1-30. doi: 10.1214/14-ba884

Milaniak, I., \& Widom, C. (2015). Does child abuse and neglect increase risk for perpetration of violence inside and outside the home?. Psychology Of Violence, 5(3), 246-255. doi: $10.1037 / a 0037956$ 
Morgan, A., Reavley, N., Ross, A., Too, L., \& Jorm, A. (2018). Interventions to reduce stigma towards people with severe mental illness: Systematic review and meta-analysis. Journal Of Psychiatric Research, 103, 120-133. doi: 10.1016/j.jpsychires.2018.05.017

Papalia, N., Ogloff, J., Cutajar, M., \& Mullen, P. (2018). Child Sexual Abuse and Criminal Offending: Gender-Specific Effects and the Role of Abuse Characteristics and Other Adverse Outcomes. Child Maltreatment, 23(4), 399-416. doi: 10.1177/1077559518785779

Parr, J., \& Pearson, D. (2019). Non-Offending Minor-Attracted Persons: Professional Practitioners' Views on the Barriers to Seeking and Receiving Their Help. Journal Of Child Sexual Abuse, 1-23. doi: $10.1080 / 10538712.2019 .1663970$

Police Service Northern Ireland (PSNI) (2018) Trends in police recorded crime in Northern Ireland 1998/1999 to 2017/2018. https://www.psni.police.uk/inside-psni/Statistics/police-recordedcrime-statistics/

Rheingold, A., Zajac, K., Chapman, J., Patton, M., de Arellano, M., Saunders, B., \& Kilpatrick, D. (2014). Child Sexual Abuse Prevention Training for Childcare Professionals: An Independent Multi-Site Randomized Controlled Trial of Stewards of Children. Prevention Science, 16(3), 374385. doi: 10.1007/s11121-014-0499-6

Richards, K. (2017). Born This Way? A Qualitative Examination of Public Perceptions of the Causes of Pedophilia and Sexual Offending against Children. Deviant Behavior, 39(7), 835-851. doi: $10.1080 / 01639625.2017 .1335526$

Scottish Government (2018) Recorded crime in Scotland 2017-2018. Edinburgh: Scottish Government. https://www.gov.scot/publications/recorded-crime-scotland-2017-18/

Stiels-Glenn, M. (2010). The availability of outpatient psychotherapy for paedophiles in Germany. Recht \& Psychiatrie, 28(2), 74-80.

Vehtari, A., Gelman, A., \& Gabry, J. (2016). Practical Bayesian model evaluation using leave-one-out cross-validation and WAIC. Statistics And Computing, 27(5), 1413-1432. doi: 10.1007/s11222016-9696-4

Walsh, K., Galea, S., \& Koenen, K. (2012). Mechanisms Underlying Sexual Violence Exposure and Psychosocial Sequelae: A Theoretical and Empirical Review. Clinical Psychology: Science And Practice, 19(3), 260-275. doi: 10.1111/cpsp.12004 
Wark, C., \& Galliher, J. (2007). Emory Bogardus and the Origins of the Social Distance Scale. The American Sociologist, 38(4), 383-395. https://doi.org/10.1007/s12108-007-9023-9

Yang, A. (2015). Building a Cognitive-Sociological Model of Stereotypes: Stereotypical Frames, Social Distance and Framing Effects. Howard Journal Of Communications, 26(3), 254-274. https://doi.org/10.1080/10646175.2015.1049757

Yuan, K., Guarnaccia, C., \& Hayslip, B. (2003). A Study Of The Distribution Of Sample Coefficient Alpha With The Hopkins Symptom Checklist: Bootstrap Versus Asymptotics. Educational And Psychological Measurement, 63(1), 5-23. doi: 10.1177/0013164402239314

10,500 people asked for help. (2019). Retrieved 6 November 2019, from https://www.dontoffend.org/story/10-500-people-asked-for-help.html 


\section{Appendix A:}

Stigma Inventory:

Please answer spontaneously and without thinking - there is no "right" or "wrong". Read the following statements and tick for each statement the answer that best suits them. Please note that the statements in the questionnaire below are for research purposes only and do not necessarily reflect the opinion of the research team.

This Questionnaire concerns individuals who (have a primary sexual interest in children/ drink large amounts of alcohol daily).

Controllability:

1: (a primary sexual interest in children / drinking large amounts of alcohol almost daily) is something you choose.

2: (a primary sexual interest in children / people who drink large amounts of alcohol almost daily) is consciously decided by the individual.

3: People have a choice whether or not they (have a primary sexual interest in children / drink large amounts of alcohol almost daily).

\section{Dangerousness:}

5: People who are (primarily sexually interested in children / drink large amounts of alcohol almost every day) are a danger to children.

6: People who are (primarily sexually interested in children / drink large amounts of alcohol almost daily) are a risk for adolescents.

7: People (who are sexually primarily interested in children / who drink large amounts of alcohol almost daily) are a risk for adults. 


\section{Appendix B:}

Social Distance:

Now try to consider the idea of having certain people involved in your life and imagine the areas of life you could see them being in and those areas you would keep them out of.

What would be your involvement with people who (have a primary sexual interest in children/ drink large amounts of alcohol daily) who have never committed a crime?

11: I would make friends with these people.

12: I would accept these people in my neighbourhood.

13: I would accept these people as work colleagues.

14: I would talk to these people.

15: These people should be locked up.

16: These people would be better dead. 


\section{Appendix C:}

Perceived Social Distance Scale

Please, state what you believe would be the "Average" British response to the following questions. Now try to consider the idea of having certain people involved in your life and imagine the areas of life you could see them being in and those areas you would keep them out of. What would be your involvement with people who (have a primary sexual interest in children/ drink large amounts of alcohol daily) who have never committed a crime?

Most people in the U.K would think:

1: "I would make friends with these people."

2: "I would accept those people in my neighbourhood."

3: "I would accept these people as work colleagues.

4: " I would talk to these people. "

5: " These people should be imprisoned. "

6: " These people would be better dead. " 


\section{Appendix D:}

Text based intervention material: Paedophilia

\section{Definition?}

It is easy to neglect the difference between a person with paedophilia and a child abuser, however the two are fundamentally different. In fact, $49 \%$ of those incarcerated for child sexual abuse are not considered to be paedophiles, and so here we can see clearly the defining point of the issue. We take it for granted that every paedophile has or will abuse a child, however an individual can be a paedophile their entire life and never commit any offence because they know that it is wrong.

The DSM-V requires that for an individual to be a paedophile they must have sexual fantasies about pre-pubescents or adolescents, it must also be the case that this fantasy is re-occurring and causes significant stress in their life.

\section{$\underline{\text { Stress? }}$}

Many people do not acknowledge that having such sexual fantasies would be a burden in the manner that it is for a non-offending paedophile. They are lonely and there is often no friend, family member, colleague or associate that they can tell about this issue for fear of being branded a child abuser. This branding is often referred to as stigmatisation, and as we know that anyone who is a paedophile is not necessarily a child abuser, we can see that stigmatising beliefs are generally unfounded, baseless opinions.

\section{Prevalence?}

In recent years professional mental health practitioners have found a growing body of evidence to suggest that there is a vastly higher number of individuals who are paedophilic but they have no desire to act upon their urges because of their reservations towards such, self-proclaimed, abhorrent behaviour. The Stop It Now campaign, designed for anonymous help over the phone regarding child sexual exploitation receives thousands of calls every month from those seeking help with paedophilic fantasies, its actually over $50 \%$ of all the calls they receive.

\section{What does that tell us?}

As much as there are those children who are at risk there is an equally sized request for help from those who have never offended but wish they could receive help in controlling what they feel. We help the victims, we rehabilitate offenders but if we made it easier for a non-offender to find help we would have less of both. The best way to protect more people is to start encouraging more non- 
offending paedophiles to be open about their desires, to get them into professional help and to end the cycle of loneliness which often leads to them creating a devastating amount of harm. 


\section{Appendix E:}

Text based intervention material: Alcoholism

Alcoholism is viewed quite differently from other mental health disorders. Many view alcoholdependent individuals are responsible for their condition, that they had a choice to become an alcoholic. This misapplication of responsibility leads to people taking a harsher and more punitive attitude to those afflicted by alcoholism, causing social rejection. Not only does this isolate alcoholics it can result in structural discrimination, wherein public rejection of these individuals reduces the opportunities available to them. Including the treatment that these individuals need.

Alcohol Use Disorder (AUD) is not something that an individual chooses to develop. Hence it could never be considered justified to punish an individual for developing AUD. The reality is that $40-60 \%$ of the risk of developing AUD is explained by genetic influences. Essentially, the risk of becoming an alcoholic is heavily influenced by your family's history of susceptibility alcoholism. This was further confirmed by the finding that alcoholism is more likely to occur in both monozygotic (identical) twins whereas it is more likely only one of a pair of dizygotic (non-identical) twins would become an alcoholic. Whilst environmental factors such as peer misuse of alcohol, positive expectations of the effect of alcohol and ineffective ways of coping with stress contribute to alcoholism none of these factors contribute as heavily as genetic predisposition.

AUD is defined as a problematic pattern of alcohol use leading to clinically significant impairment or distress, as manifested by at least two of the following, occurring within a 12-month period:

1. Alcohol is often taken in larger amounts or over a longer period than was intended.

2. There is a persistent desire or unsuccessful efforts to cut down or control alcohol use.

3. A great deal of time is spent in activities necessary to obtain alcohol, use alcohol, or recovery from its effects.

4. Craving, or a strong desire or urge to use alcohol.

5. Recurrent alcohol use resulting in a failure to fulfil major role obligations at work, school, or home.

6. Continued alcohol use despite having persistent or recurrent social or interpersonal problems caused or exacerbated by the effects of alcohol.

7. Important social, occupational, or recreational activities are given up or reduced because of alcohol use. 
8. Recurrent alcohol use in situations in which it is physically hazardous.

9. Alcohol use is continued despite knowledge of having a persistent or recurrent physical or psychological problem that is likely to have been caused or exacerbated by alcohol.

The chronic and repeated use of alcohol can damage the gastrointestinal tract, cardiovascular system, and the central and peripheral nervous systems. Around 15\% of severe AUD patients develop liver cirrhosis or pancreatitis. The Scottish health survey this year found 1 in 4 scots drank a harmful amount of alcohol weekly. In 2015 alcohol was the primary factor in just over 1 in 15 deaths in Scotland $(3,705), 1,048$ of which were due to cancers formed from alcohol misuse.

AUD is not a choice; it is a biologically based addiction that causes a great deal of harm. 
Appendix F:

"How I Overcame Alcoholism"

https://www.youtube.com/watch?v=cQ-qsOAjBI0\&t=2s

"Inside The Life Of A 'Virtuous' Paedophile"-shortened version used in study

https://www.youtube.com/watch?v=iCN1ksgxw1g\&t=2s 


\section{Appendix G:}

Thank you for participating in this online experiment.

As you were aware from the beginning of the experiment there were two groups of people we wanted opinions on but you were only asked about your opinion on one, the two groups were alcoholics and non-offending paedophiles.

\section{The aims of this experiment are to:}

- gather information on the current public opinion towards alcoholism and non-offending paedophiles,

- assess how well these opinions can be changed using educational text and counter-stereotypical examples portrayed within video material,

- examine how well the questionnaires used in this experiment capture the relevant information about opinions towards these two groups of individuals.

\section{Our Hypotheses:}

We hypothesised that there would be a reduction in stigmatising opinions following the interventions for both groups and that stigmatising opinions would be greater towards the nonoffending paedophile group than the alcoholic group.

\section{Why the Study is Needed:}

The reason for this experiment is that research has highlighted that those in prison for child sexual abuse are not representative of paedophilic disorder and that this disorder affects far more people than initially believed. The current issue is that whilst we now know that most people with paedophilic disorder will never offend there is little in the way of support for such individuals to receive help which would alleviate their distress and additionally enable a greater safeguard against potential offending. Professional formal help for this disorder is available from the NHS but there is a disproportionately high number of individuals seeking help from informal anonymous sources compared to the number of those engaging the formal help available from the NHS. For instance, over half of the calls, around 35,000, to the anonymous help line Stop It Now are from non-offending paedophiles. This difference in demand has been hypothesised as the result of the anonymity of the Stop It Now! help line which allows non-offenders to seek help whilst ensuring they do not experience stigma, from public or professional bodies. If we want non-offending paedophiles to seek formal professional help, we need to find a way of addressing stigmatism. Which led to this research 
addressing how a stigmatism intervention would work for the purpose of changing public opinion, ultimately to make seeking help for paedophilic disorder more acceptable.

If you wish to see the results of this experiment feel free to email the researchers and we will provide you access to the final research paper online.

Additionally, if you have any comments about the content or any aspects of this experiment please feel free to make them in the comment box below.

Once again thank you for your participation.

Ross Keegan, ross.keegan@aol.com

Guillaume Rousselet, guillaume.rousselet@glasgow.ac.uk 


\section{Appendix H:}

Priors: <- c(set_prior("normal(0, 1)", class = "b", coef = "condition1"),

set_prior("normal(0, 0.5)", class = "b", coef = "Intervention1"),

set_prior("normal(0, 0.5)", class = "b", coef = "condition1:Intervention1"),

set_prior("cauchy $(0,1) "$, class = "sd"))

Note. Prior values based on Jahnke, Imhoff \& Hoyer (2014) results.

Model 1 Formula: (Score condition*Intervention + (1 | Id))

Model 2 Formula: $($ Score $\sim$ condition*Intervention $+(1+$ Intervention $\mid$ Id $)+(1+$ condition | Item $)$

Model 3 Formula: $($ Score $~$ condition*Intervention $+(1+$ Intervention $\mid$ Id $)+(1+$ condition + Intervention +condition:Intervention| Item)

Model 4 Formula: (Score condition*Intervention $+(1+$ Intervention $\mid$ condition $)+(1+$ Intervention $\mid \mathrm{Id})+(1+$ condition + Intervention +condition:Intervention | Item))

Stigma Inventory Models Leave One Out Information Criterion Estimates and Standard Error

\begin{tabular}{lcc} 
& Estimate & SE \\
\hline Model[1] & 3191.754 & 30.73217 \\
Model [2] & 3191.037 & 30.51152 \\
Model [3] & 3190.756 & 30.57128 \\
Model [4] & 3194.190 & 30.60760 \\
\hline
\end{tabular}

Social Distance Scale Models Leave One Out Information Criterion Estimates and Standard Error Estimate SE

\begin{tabular}{lll} 
Model [1] & 3267.364 & 28.13627 \\
Model [2] & 3266.126 & 28.00900 \\
Model [3] & 3066.380 & 36.87017 \\
Model [4] & 3043.245 & 37.59307 \\
\hline
\end{tabular}


Social Distance Scale Models Leave One Out Information Criterion Estimates and Standard Error Estimate SE

Model [1] $3374.773 \quad 19.61802$

Model [2] $\quad 3374.383 \quad 19.52750$

Model [3] $3176.709 \quad 31.31009$

Model [4] $3172.856 \quad 31.64478$ 


\section{Appendix I}

\section{Stigmatism Inventory Estimates}

Formula: Score $~$ condition * Intervention + (1 + Intervention | Id $)+(1+$ condition + Intervention + condition:Intervention | Item $)$

Samples: 4 chains, each with iterations $=4000 ;$ warmup $=2000 ;$ thin $=1$;

total post-warmup samples $=8000$

\section{Table 1}

Stigmatism Inventory Participant Group-Level Effects

Estimate Est.Error $1-95 \% \mathrm{Cl} \quad$ u-95\% Cl Rhat Bulk ESS Tail ESS

\begin{tabular}{|c|c|c|c|c|c|c|c|}
\hline sd(Intercept) & 0.54 & 0.07 & 0.40 & 0.70 & 1.00 & 2964 & 5351 \\
\hline sd(Intervention1) & 0.26 & 0.15 & 0.01 & 0.55 & 1.01 & 605 & 1528 \\
\hline cor(Intercept,Int & n1) & .10 & 0.39 & -0.75 & 0.79 & 1.00 & 2930 \\
\hline
\end{tabular}

Note. Number of levels:80 
Table 2

Stigmatism Inventory Item Group-Level Effects

Estimate Est.Error I-95\% Cl u-95\% Cl Rhat Bulk ESS Tail ESS

\begin{tabular}{lcccccccccc} 
sd(Intercept) & 0.07 & 0.06 & 0.00 & 0.23 & 1.00 & 4985 & 3899 & \\
sd(condition1) & 0.23 & 0.16 & 0.02 & 0.64 & 1.00 & 2706 & 2602 & \\
sd(Intervention1) & 0.09 & 0.08 & 0.00 & 0.28 & 1.00 & 5012 & 4745 & \\
sd(condition1:Intervention1) & 0.15 & 0.13 & 0.01 & 0.47 & 1.00 & 4567 & 3939 & \\
cor(Intercept,condition1) & -0.04 & 0.45 & -0.83 & 0.79 & 1.00 & 3955 & 5047 & \\
cor(Intercept, Intervention1) & -0.08 & 0.45 & -0.85 & 0.80 & 1.00 & 8421 & 5197 & \\
cor(condition1,Intervention1) & 0.04 & 0.45 & -0.80 & 0.83 & 1.00 & 7869 & 6296 \\
cor(Intercept,condition1:Intervention1) & -0.00 & 0.45 & -0.80 & 0.82 & 1.00 & 8552 & 6042 \\
cor(condition1,condition1:Intervention1) & -0.13 & 0.45 & -0.87 & 0.76 & 1.00 & 7169 & 5630 \\
cor(Intervention1,condition1:Intervention1) & -0.10 & 0.45 & -0.85 & 0.78 & 1.00 & 5641 & 5961 \\
\hline
\end{tabular}

Note. Number of levels: 6 
Table 3

Stigmatism Inventory Population-Level Effects

Estimate Est.Error $1-95 \% \mathrm{Cl}$ u-95\% Cl Rhat Bulk ESS Tail ESS

\begin{tabular}{lcccccccc} 
Intercept[1] & -0.73 & 0.12 & -0.97 & -0.51 & 1.00 & 3341 & 4953 \\
Intercept[2] & -0.07 & 0.12 & -0.30 & 0.15 & 1.00 & 3509 & 4744 \\
Intercept[3] & 0.61 & 0.12 & 0.38 & 0.84 & 1.00 & 3582 & 5539 \\
Intercept[4] & 1.18 & 0.12 & 0.95 & 1.42 & 1.00 & 3802 & 5559 \\
Intercept[5] & 1.86 & 0.13 & 1.61 & 2.12 & 1.00 & 3953 & 4753 \\
condition1 & 0.44 & 0.18 & 0.09 & 0.81 & 1.00 & 2747 & 3072 \\
Intervention1 & 0.09 & 0.11 & -0.13 & 0.30 & 1.00 & 5691 & 5573 \\
condition1:Intervention1 & -0.20 & 0.16 & -0.52 & 0.12 & 1.00 & 5402 & 5465 \\
\hline
\end{tabular}




\section{Social Distance Scale Estimates}

Formula: Score condition * Intervention + (1 + Intervention | Id $)+(1+$ condition + Intervention + condition:Intervention | Item) Samples: 4 chains, each with iter $=4000$; warmup $=2000$; thin $=1$;

total post-warmup samples $=8000$

Table 4

Social Distance Scale Participant Group-level Estimates

Estimate Est.Error $1-95 \% \mathrm{Cl}$ u-95\% $\mathrm{Cl}$ Rhat Bulk ESS Tail ESS

\begin{tabular}{lccccccc} 
sd(Intercept) & 0.58 & 0.07 & 0.44 & 0.73 & 1.00 & 3495 & 5413 \\
sd(Intervention1) & 0.20 & 0.12 & 0.01 & 0.44 & 1.00 & 1048 & 2029 \\
cor(Intercept,Intervention1) & 0.30 & 0.41 & -0.62 & 0.95 & 1.00 & 4220 & 3788 \\
\hline
\end{tabular}

Note. Number of levels:80 
Table 5

Social Distance Scale Item Group-level Estimates

Estimate Est.Error l-95\% Cl u-95\% Cl Rhat Bulk ESS Tail ESS

\begin{tabular}{lccccccc} 
sd(Intercept) & 0.61 & 0.20 & 0.34 & 1.11 & 1.00 & 3468 & 4449 \\
sd(condition1) & 0.42 & 0.19 & 0.15 & 0.88 & 1.00 & 4667 & 5384 \\
sd(Intervention1) & 0.41 & 0.20 & 0.11 & 0.90 & 1.00 & 3966 & 3051 \\
sd(condition1:Intervention1) & 0.79 & 0.29 & 0.39 & 1.47 & 1.00 & 4246 & 4701 \\
cor(Intercept,condition1) & -0.63 & 0.27 & -0.96 & 0.08 & 1.00 & 5000 & 6054 \\
cor(Intercept,Intervention1) & 0.39 & 0.34 & -0.36 & 0.91 & 1.00 & 5824 & 5776 \\
cor(condition1,Intervention1) & -0.32 & 0.36 & -0.89 & 0.45 & 1.00 & 6241 & 6197 \\
cor(Intercept,condition1:Intervention1) & -0.61 & 0.27 & -0.96 & 0.07 & 1.00 & 5020 & 5953 \\
cor(condition1,condition1:Intervention1) & 0.31 & 0.35 & -0.44 & 0.88 & 1.00 & 6244 & 6571 \\
cor(Intervention1,condition1:Intervention1) & -0.41 & 0.33 & -0.90 & 0.36 & 1.00 & 6218 & 6634 \\
\hline
\end{tabular}

Note. Number of levels:6 
Table 6

Social Distance Scale Population-level Estimates

Estimate Est.Error l-95\% Cl u-95\% Cl Rhat Bulk ESS Tail ESS

\begin{tabular}{lccccccc} 
Intercept[1] & -1.55 & 0.27 & -2.08 & -0.971 .00 & 2413 & 3316 \\
Intercept[2] & -0.85 & 0.27 & -1.37 & -0.281 .00 & 2433 & 3352 \\
Intercept[3] & -0.12 & 0.27 & -0.64 & 0.441 .00 & 2449 & 3296 \\
Intercept[4] & 0.45 & 0.27 & -0.07 & 1.001 .00 & 2493 & 3318 \\
Intercept[5] & 1.04 & 0.27 & 0.53 & 1.611 .00 & 2501 & 3499 \\
condition1 & -0.26 & 0.24 & -0.70 & 0.211 .00 & 3156 & 3751 \\
Intervention1 & 0.19 & 0.18 & -0.17 & 0.541 .00 & 5168 & 5096 \\
condition1:Intervention1 & 0.08 & 0.28 & -0.49 & 0.641 .00 & 4918 & 5291 \\
\hline
\end{tabular}




\section{Perceived Social Distance Scale Estimates}

Formula: Score $\sim$ condition * Intervention $+(1+$ Intervention | Id $)+(1+$ condition + Intervention + condition:Intervention | Item $)$

Samples: 4 chains, each with iter $=4000 ;$ warmup $=2000$; thin $=1$;

total post-warmup samples $=8000$

\section{Table 7}

Perceived Social Distance Scale Participants Group-level Estimates

Estimate Est.Error I-95\% Cl u-95\% Cl Rhat Bulk ESS Tail ESS

\begin{tabular}{lllllll} 
sd(Intercept) & 0.39 & 0.06 & 0.28 & 0.511 .00 & 3619 & 5152 \\
sd(Intervention1) & 0.08 & 0.06 & 0.00 & 0.221 .00 & 3491 & 4002 \\
cor(Intercept,Intervention1) & 0.12 & 0.54 & -0.91 & 0.951 .00 & 7733 & 5233 \\
\hline
\end{tabular}

Note. Number of levels:80 
Table 8

Perceived Social Distance Scale Item Group-level Estimates

Estimate Est.Error $1-95 \% \mathrm{Cl} \quad$ u-95\% Cl Rhat Bulk ESS Tail ESS

\begin{tabular}{llllllll}
\hline sd(Intercept) & 0.77 & 0.26 & 0.43 & 1.39 & 1.00 & 2872 & 3729 \\
sd(condition1) & 0.90 & 0.30 & 0.50 & 1.64 & 1.00 & 3288 & 3995 \\
sd(Intervention1) & 0.21 & 0.16 & 0.01 & 0.60 & 1.00 & 3237 & 3748 \\
sd(condition1:Intervention1) & 0.35 & 0.24 & 0.02 & 0.91 & 1.00 & 2930 & 2923 \\
cor(Intercept,condition1) & -0.62 & 0.26 & -0.94 & 0.04 & 1.00 & 3390 & 4205 \\
cor(Intercept,Intervention1) & 0.02 & 0.40 & -0.70 & 0.77 & 1.00 & 7351 & 5521 \\
cor(condition1,Intervention1) & -0.24 & 0.39 & -0.87 & 0.58 & 1.00 & 8515 & 6424 \\
cor(Intercept,condition1:Intervention1) & -0.26 & 0.38 & -0.87 & 0.56 & 1.00 & 6997 & 5417 \\
cor(condition1,condition1:Intervention1) & -0.00 & 0.39 & -0.69 & 0.74 & 1.00 & 7229 & 5710 \\
cor(Intervention1,condition1:Intervention1) & -0.03 & 0.43 & -0.81 & 0.79 & 1.00 & 5726 & 6542 \\
\hline
\end{tabular}

Note. Number of levels:6 
Table 9

Perceived Social Distance Scale Population-level Estimates

Estimate Est.Error I-95\% Cl u-95\% Cl Rhat Bulk ESS Tail ESS

\begin{tabular}{lccccccc} 
Intercept[1] & -1.29 & 0.32 & -1.94 & -0.65 & 1.00 & 2173 & 3056 \\
Intercept[2] & -0.47 & 0.32 & -1.11 & 0.16 & 1.00 & 2166 & 3284 \\
Intercept[3] & 0.05 & 0.32 & -0.59 & 0.68 & 1.00 & 2167 & 3245 \\
Intercept[4] & 0.47 & 0.32 & -0.18 & 1.11 & 1.00 & 2170 & 2951 \\
Intercept[5] & 1.36 & 0.32 & 0.71 & 2.01 & 1.00 & 2199 & 3200 \\
condition1 & 0.09 & 0.37 & -0.66 & 0.83 & 1.00 & 2398 & 3487 \\
Intervention1 & 0.08 & 0.13 & -0.20 & 0.34 & 1.00 & 5210 & 4514 \\
condition1:Intervention1 1 & -0.19 & 0.19 & -0.56 & 0.21 & 1.00 & 4548 & 4822 \\
\hline
\end{tabular}




\section{Appendix J}

PCA and EFA Cumulative Variance Explained by Factors for Each Stigmatism Scale

\begin{tabular}{|c|c|c|c|c|}
\hline Condition & Scale & Test & Factors & Cumulative variance \\
\hline$A U D$ & Stigma Inventory & EFA & 2 & 0.5041845 \\
\hline$A U D$ & Stigma Inventory & PCA & 2 & 0.6418919 \\
\hline$A U D$ & Social Distance & EFA & 1 & 0.2921918 \\
\hline$A U D$ & Social Distance & EFA & 2 & 0.3963764 \\
\hline$A U D$ & Social Distance & PCA & 1 & 0.3823111 \\
\hline$A U D$ & Social Distance & PCA & 2 & 0.5798710 \\
\hline$A U D$ & $\begin{array}{l}\text { Perceived Social } \\
\text { Distance }\end{array}$ & EFA & 1 & 0.304371 \\
\hline$A U D$ & $\begin{array}{l}\text { Perceived Social } \\
\text { Distance }\end{array}$ & EFA & 2 & 0.5262950 \\
\hline$A U D$ & $\begin{array}{l}\text { Perceived Social } \\
\text { Distance }\end{array}$ & PCA & 1 & 0.5200778 \\
\hline$A U D$ & $\begin{array}{l}\text { Perceived Social } \\
\text { Distance }\end{array}$ & PCA & 2 & 0.6594677 \\
\hline$P W P$ & Stigma Inventory & EFA & 2 & 0.5181721 \\
\hline$P W P$ & Stigma Inventory & PCA & 2 & 0.6335692 \\
\hline$P W P$ & Social Distance & EFA & 1 & 0.3790375 \\
\hline$P W P$ & Social Distance & EFA & 2 & 0.5711277 \\
\hline$P W P$ & Social Distance & PCA & 1 & 0.4490941 \\
\hline$P W P$ & Social Distance & PCA & 2 & 0.6736570 \\
\hline$P W P$ & $\begin{array}{l}\text { Perceived Social } \\
\text { Distance }\end{array}$ & EFA & 1 & 0.4314465 \\
\hline$P W P$ & $\begin{array}{l}\text { Perceived Social } \\
\text { Distance }\end{array}$ & EFA & 2 & 0.6078615 \\
\hline$P W P$ & $\begin{array}{l}\text { Perceived Social } \\
\text { Distance }\end{array}$ & PCA & 1 & 0.5200778 \\
\hline$P W P$ & $\begin{array}{l}\text { Perceived Social } \\
\text { Distance }\end{array}$ & PCA & 2 & 0.7150653 \\
\hline
\end{tabular}




\section{Appendix K}

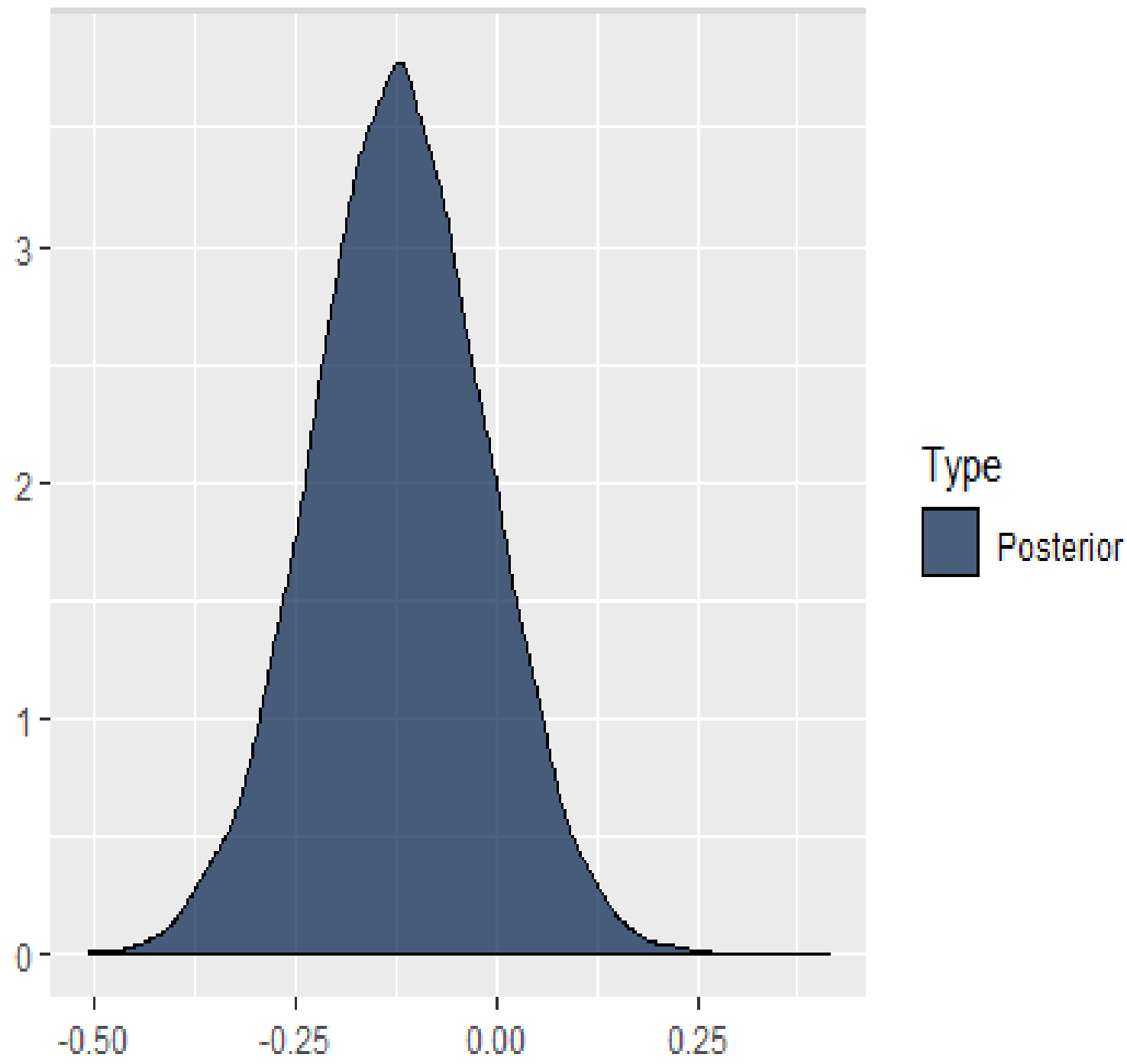

Figure 1

Stigma Inventory Hypothesis 1 Posterior Distribution

Note. Formula: "(condition1 + Intervention1 + condition1:Intervention1) < (condition1)" Tests the hypothesis that the estimate for the post-intervention PWP group will be lower than the preintervention PWP group 


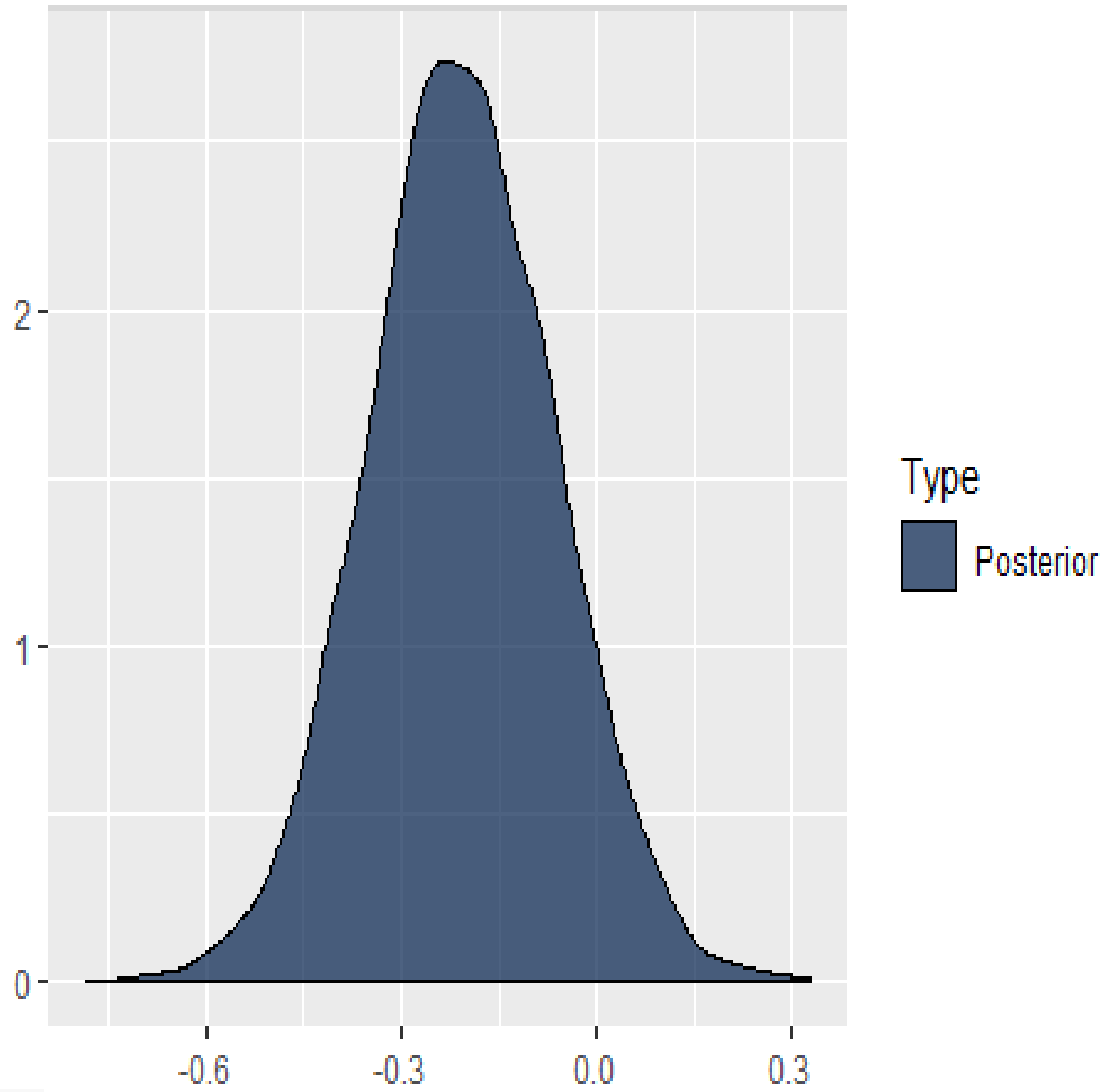

Figure 2

Stigma Inventory Hypothesis 2 Posterior Distribution

Note. Formula: "(Intervention1 + condition1:Intervention1) < (Intervention1)" Tests the hypothesis that estimates for the intervention will be lower for the PWP condition over the AUD condition, which would indicate that the intervention effect has a greater propensity to decrease stigmatism score 


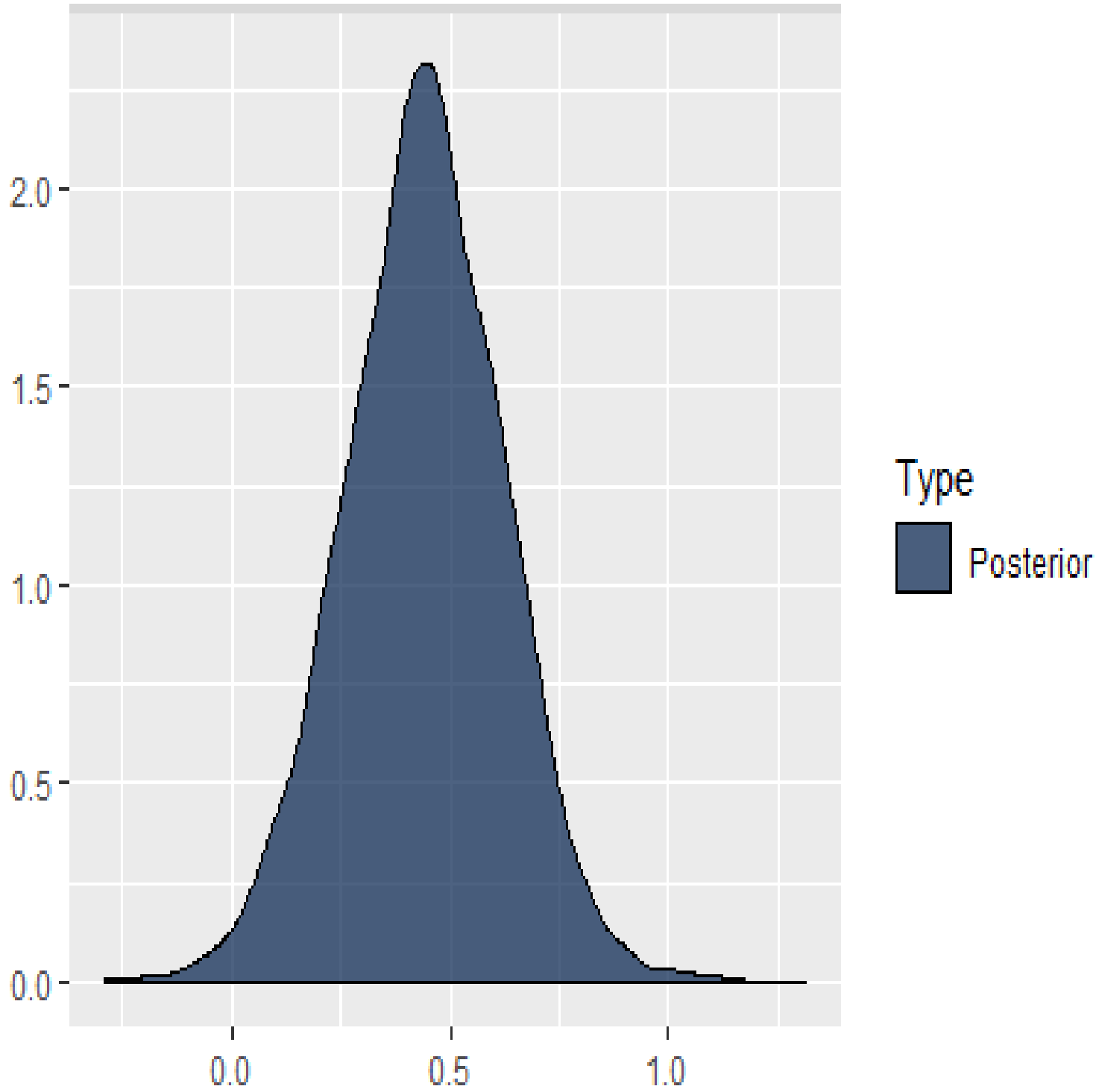

Figure 3

Stigma Inventory Hypothesis 3 Posterior Distribution

Note. Formula: "condition $1>0$ " This formulation tests that the estimates for condition 1 is greater than 0 , which is equivalent to testing if the PWP estimate is greater than the AUD estimate formalised as: condition $1>$ condition $1 * 0$. 


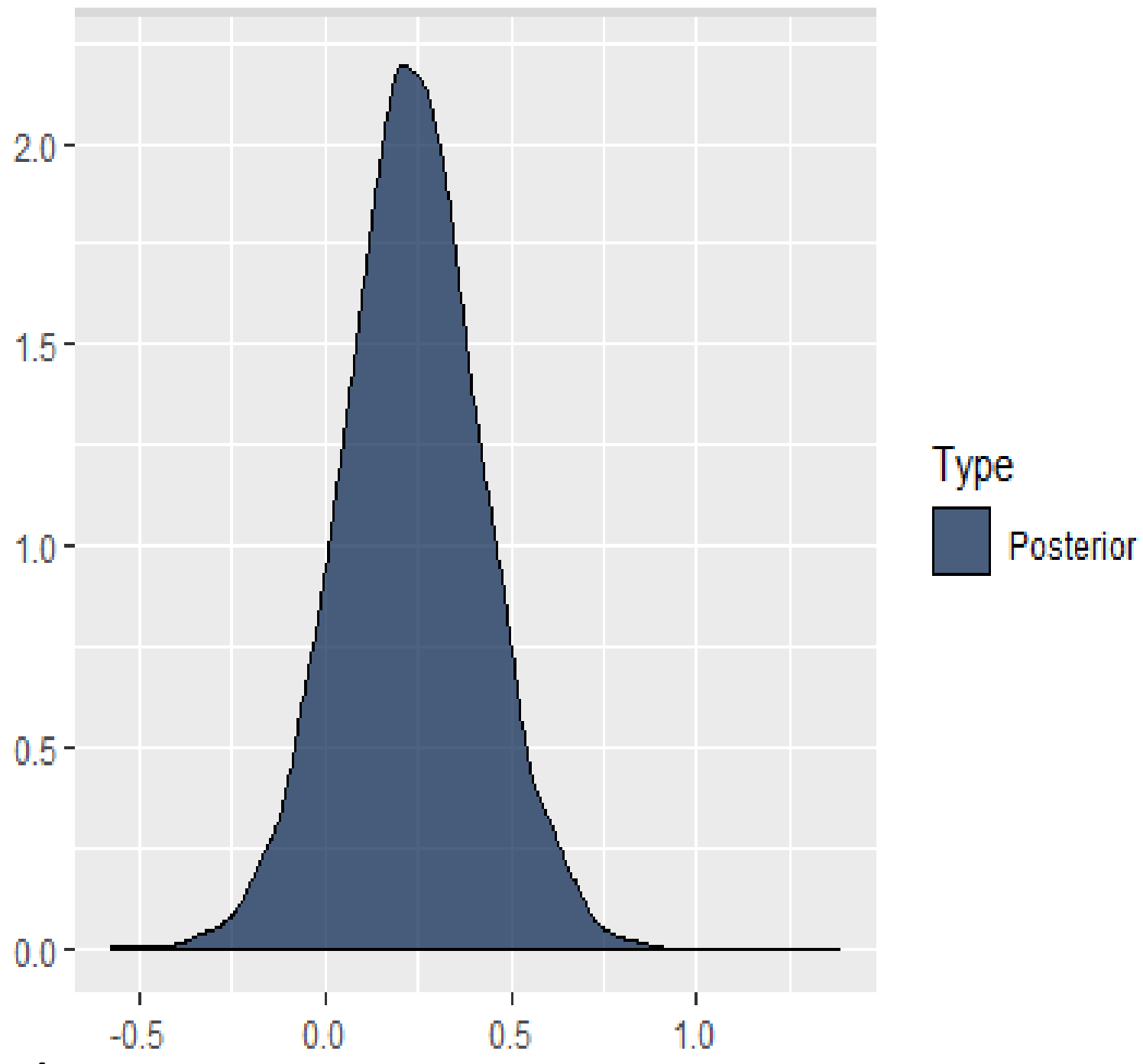

Figure 4

Stigma Inventory Hypothesis 4 Posterior Distribution

Note. Formula: "(condition1 + Intervention1 + condition1:Intervention1) > (Intervention1)" This tests the hypothesis that the estimate for the PWP group post-intervention will be greater than the AUD condition post-intervention. 


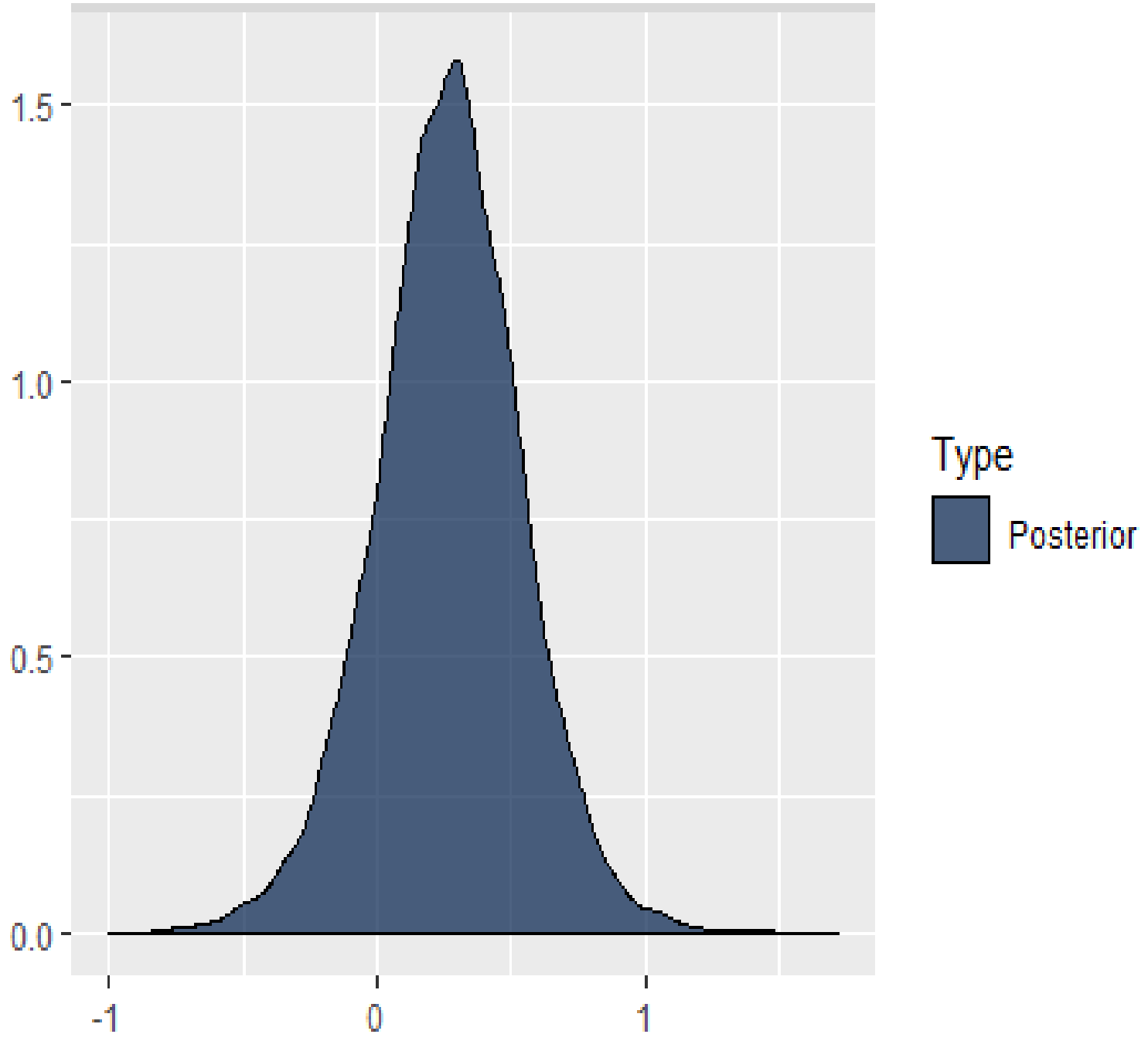

Figure 5

Social Distance Scale Hypothesis 1 Posterior Distribution

Note. Formula: "(condition1 + Intervention1 + condition1:Intervention1) < (condition1)" Tests the hypothesis that the estimate for the post-intervention PWP group will be lower than the preintervention PWP group 


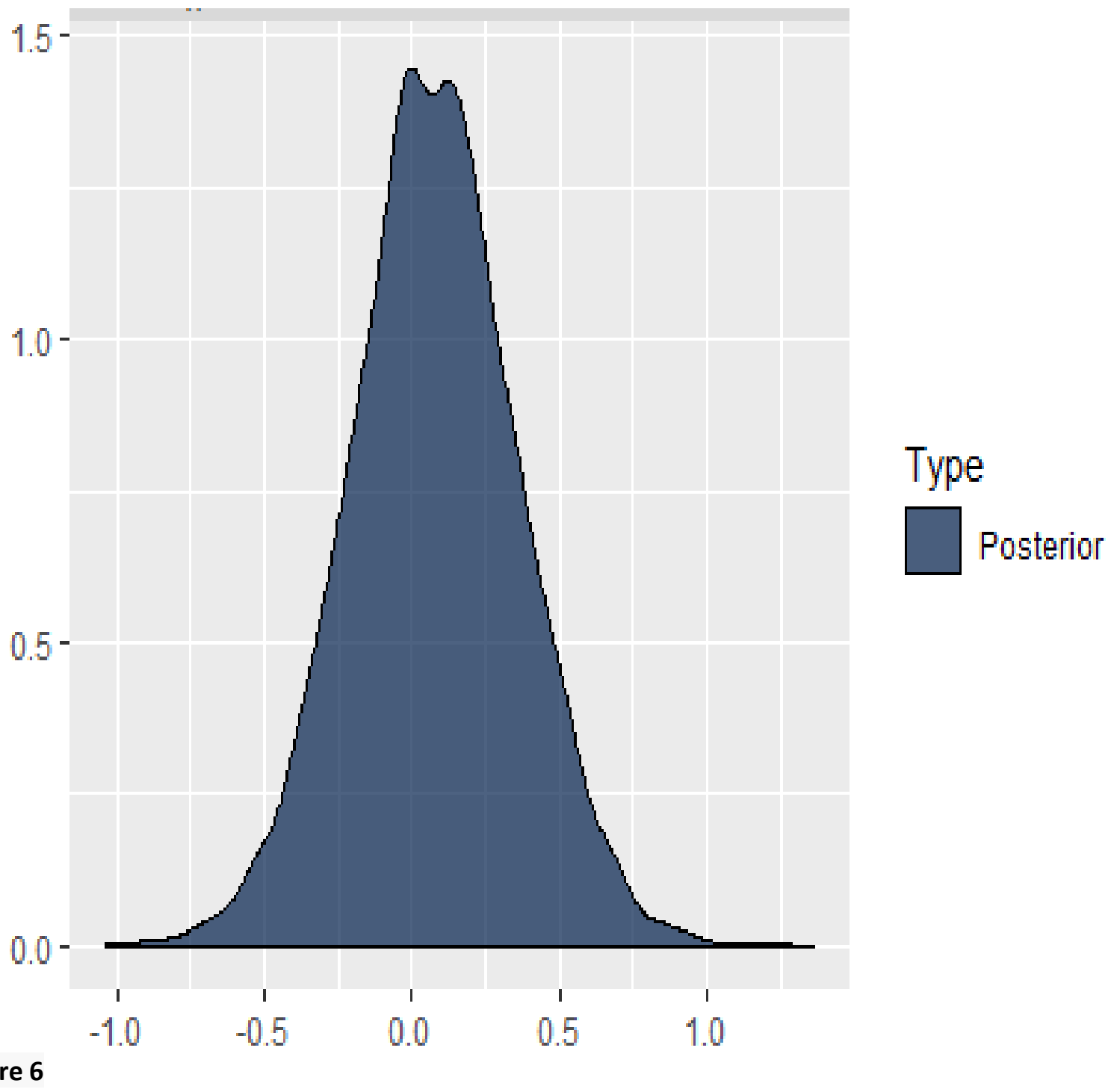

Social Distance Scale Hypothesis 2 Posterior Distribution

Note. Formula: "(Intervention1 + condition1:Intervention1) < (Intervention1)" Tests the hypothesis that estimates for the intervention will be lower for the PWP condition over the AUD condition, which would indicate that the intervention effect has a greater propensity to decrease stigmatism scores 


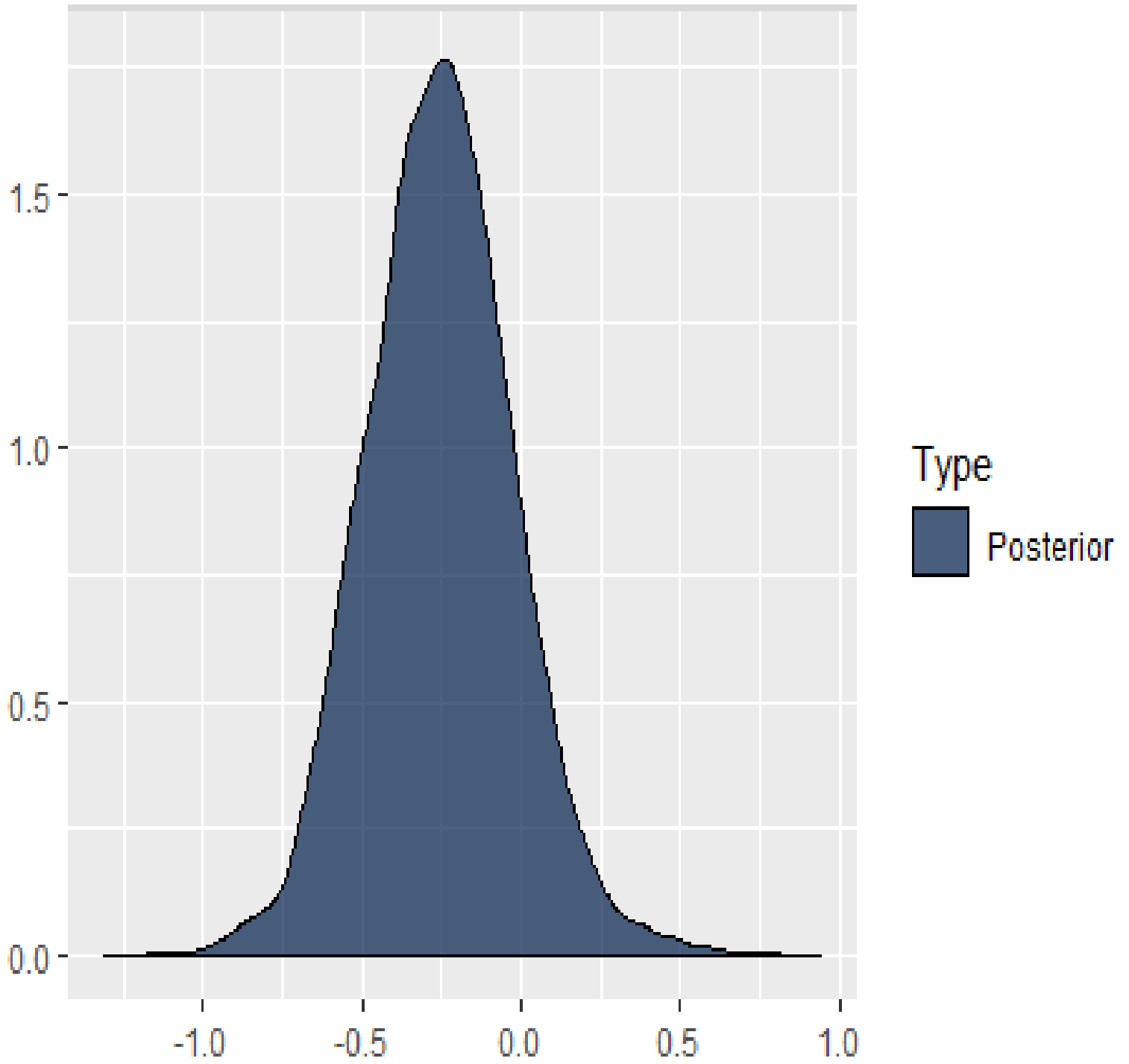

Figure 7

Social Distance Scale Hypothesis 3 Posterior Distribution

Note. Formula: "condition $1>0$ " This formulation tests that the estimates for condition 1 is greater than 0 , which is equivalent to testing if the PWP estimate is greater than the AUD estimate formalised as: condition $1>$ condition $1 * 0$. 


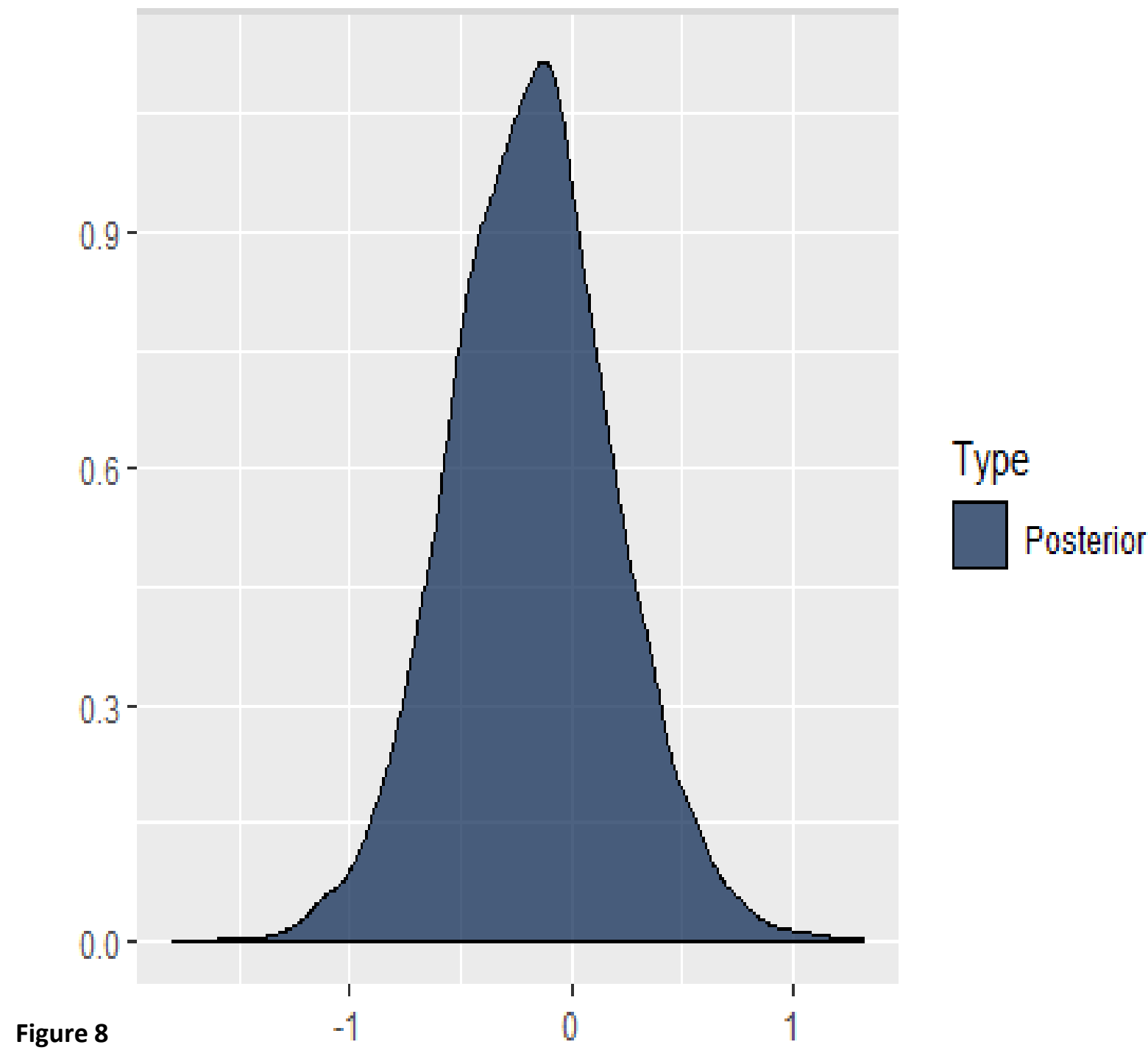

Social Distance Scale Hypothesis 4 Posterior Distribution

Note. Formula: "(condition1 + Intervention1 + condition1:Intervention1) > (Intervention1)" This tests the hypothesis that the estimate for the PWP group post-intervention will be greater than the AUD condition post-intervention. 


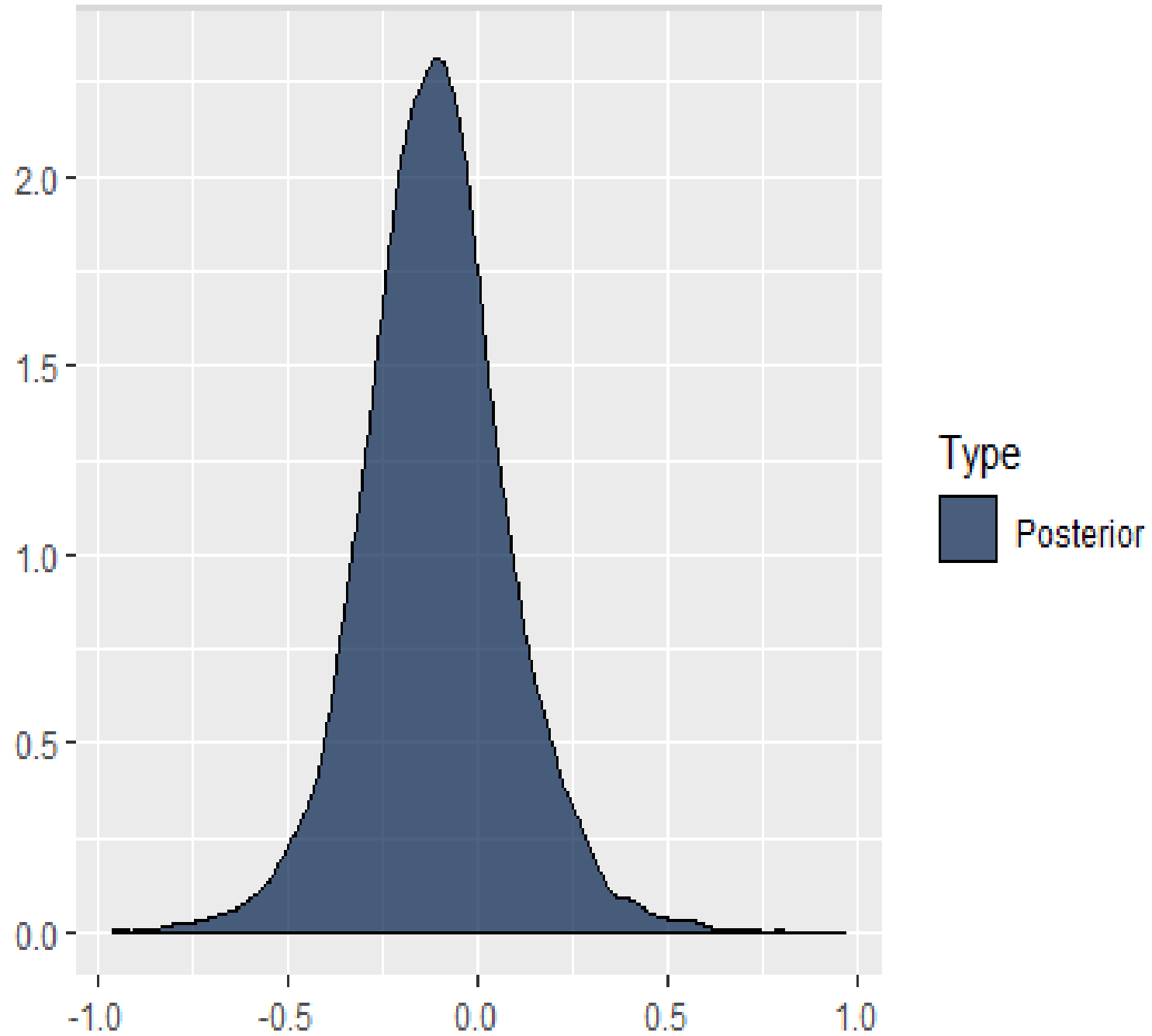

Figure 9

Perceived Social Distance Scale Hypothesis 1 Posterior Distribution

Note. Formula: "(condition1 + Intervention1 + condition1:Intervention1) < (condition1)" Tests the hypothesis that the estimate for the post-intervention PWP group will be lower than the preintervention PWP group 


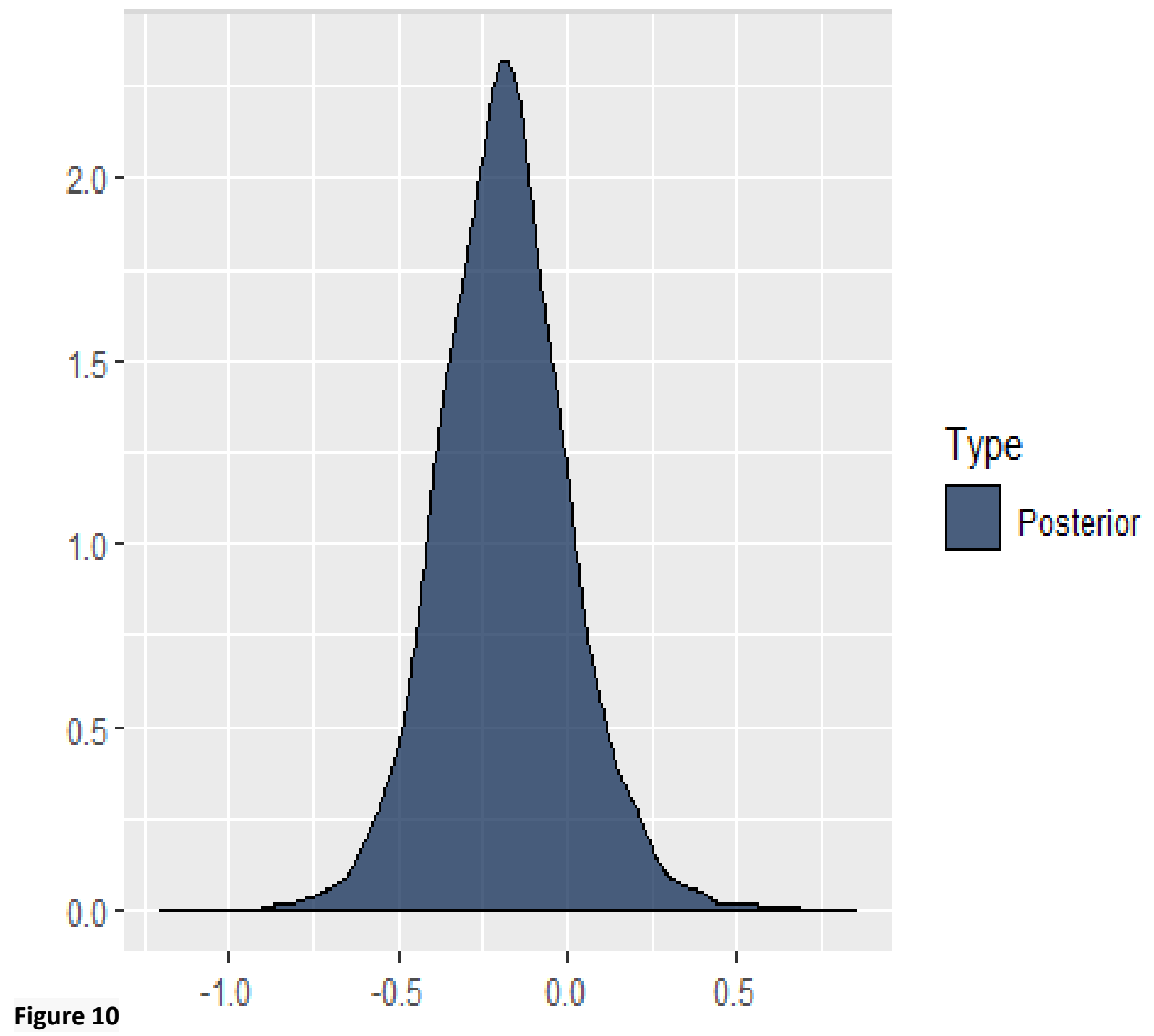

Perceived Social Distance Scale Hypothesis 2 Posterior Distribution

Note. Formula: "(Intervention1 + condition1:Intervention1) < (Intervention1)" Tests the hypothesis that estimates for the intervention will be lower for the PWP condition over the AUD condition, which would indicate that the intervention effect has a greater propensity to decrease scores 


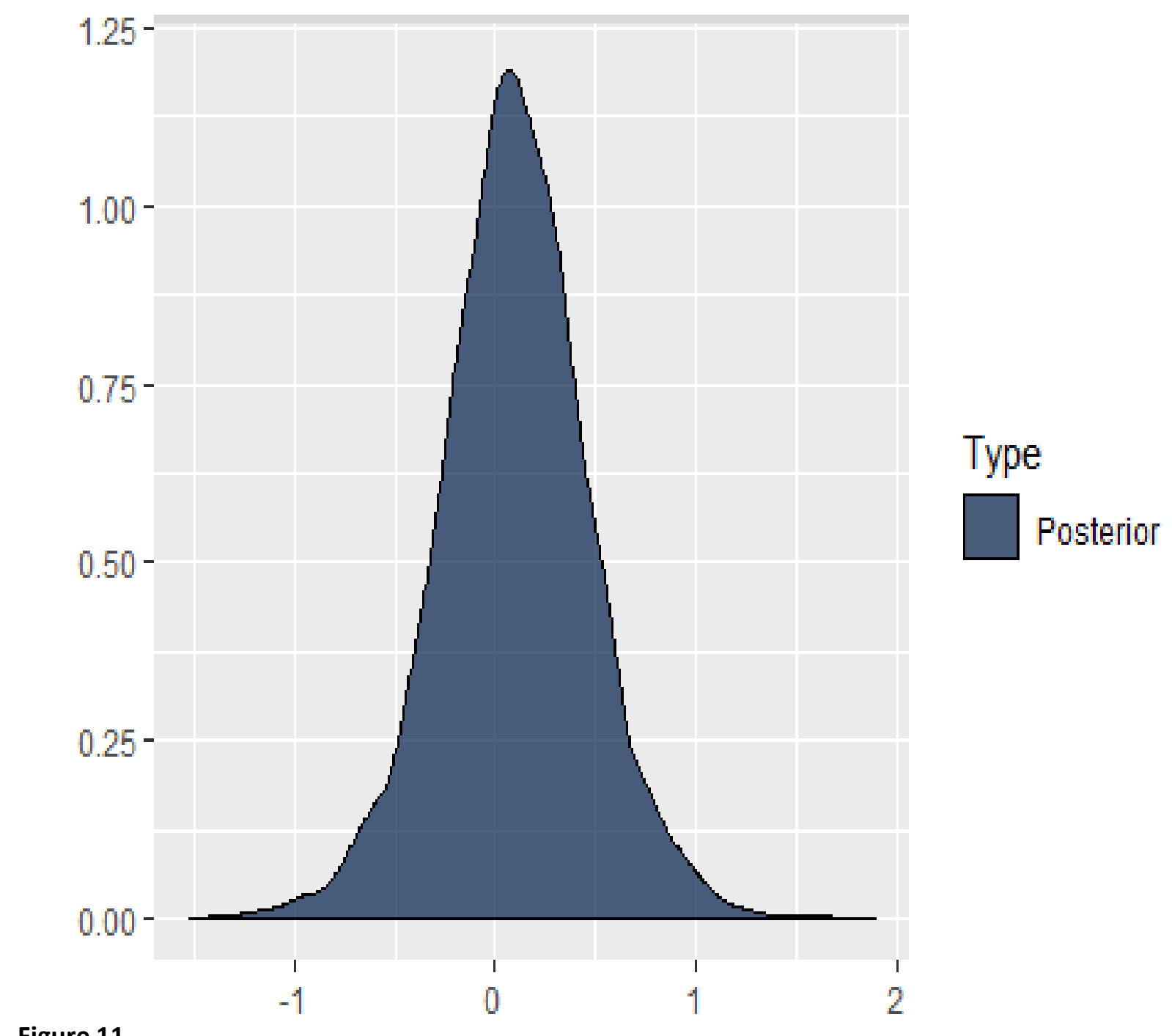

Figure 11

Perceived Social Distance Scale Hypothesis 3 Posterior Distribution

Note. Formula: "condition $1>0$ " This formulation tests that the estimates for condition 1 is greater than 0 , which is equivalent to testing if the PWP estimate is greater than the AUD estimate formalised as: condition $1>$ condition $1 * 0$. 


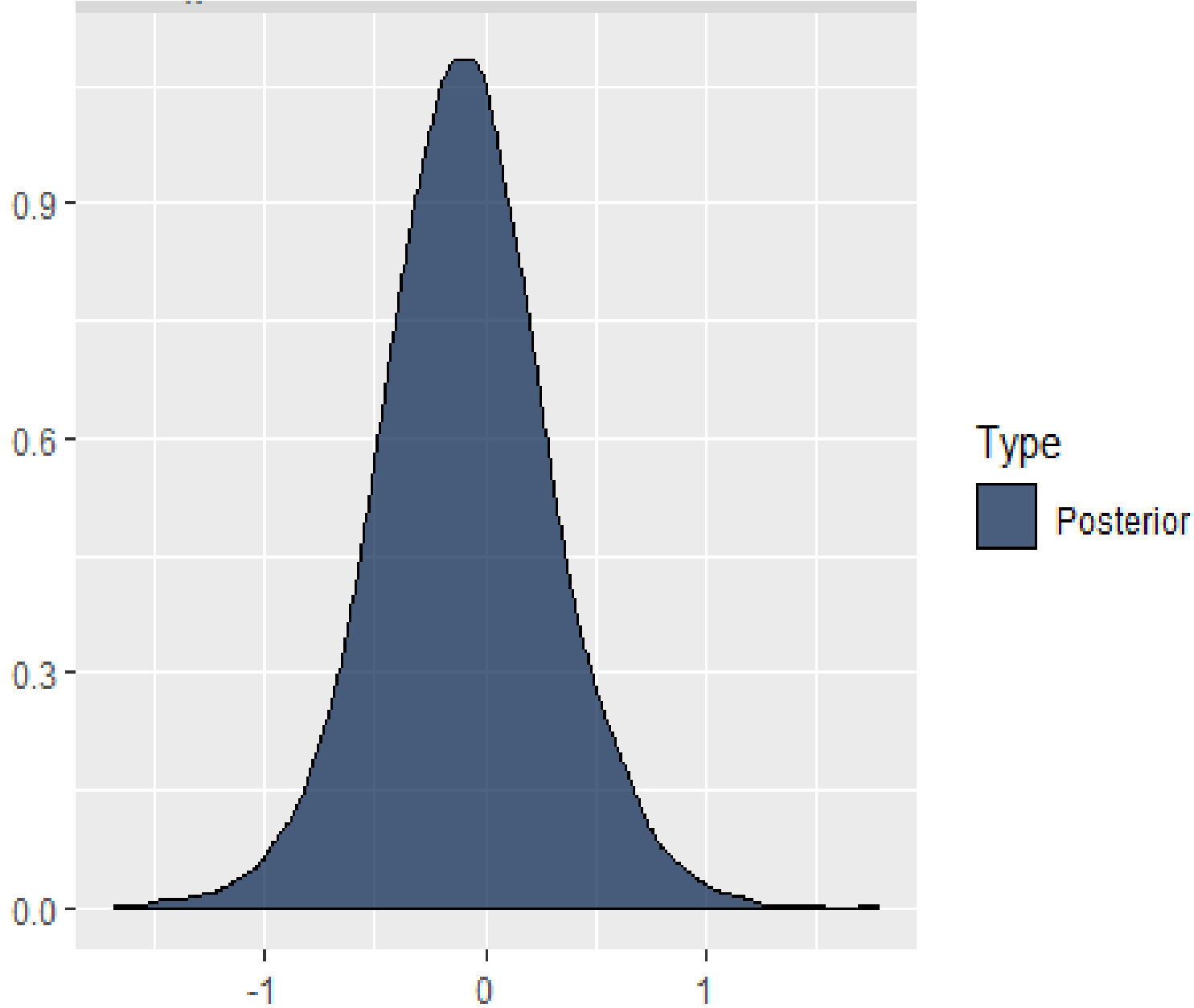

Figure 12

\section{Perceived Social Distance Scale Hypothesis 4 Posterior Distribution}

Note. Formula: "(condition1 + Intervention1 + condition1:Intervention1) > (Intervention1)" This tests the hypothesis that the estimate for the PWP group post-intervention will be greater than the AUD condition post-intervention. 